\title{
SATURN PLASMA SOURCES AND ASSOCIATED TRANSPORT PROCESSES
}

M. Blanc ${ }^{1}$, D. J. Andrews ${ }^{2}$, A. J. Coates ${ }^{3}$, D. C. Hamilton ${ }^{4}$, C.M. Jackman ${ }^{5}$, X. Jia, ${ }^{6}$ A. Kotova ${ }^{7}$, M. Morooka ${ }^{8}$, H. T. Smith9, and J. H. Westlake ${ }^{9}$

Affiliations:

1. IRAP, CNRS/Université Toulouse III, Toulouse, France

2. Swedish Institute for Space Physics (Uppsala), Sweden

3. Mullard Space Science Laboratory, University College

London, Holmbury St Mary, Dorking RH5 6NT, UK

4. Department of Physics, University of Maryland, College Park, MD 20742, USA

5. School of Physics and Astronomy, University of Southampton, Southampton, S017 1BJ, UK

6. Department of Atmospheric, Oceanic, and Space Sciences, University of Michigan, Ann Arbor, MI 48109-2143, USA

7. Max Planck Institute for Solar System Research, Göttingen, Germany; IRAP, CNRS/Université Paul Sabatier Toulouse III, Toulouse, France

8. LASP, University of Colorado at Boulder, CO, USA

9. Johns Hopkins University Applied Physics Laboratory, Laurel, MD, USA

\section{Introduction}

The magnetosphere of Saturn is not observable from the surface of the Earth, because its main radio emission frequencies are below the terrestrial ionospheric cut-off. Therefore, most of what we know about the kronian magnetosphere was revealed to us by the investigations conducted by space probes, first Pioneer, Voyager and then Cassini. The first picture of Saturn's magnetosphere emerged from the plasma measurements performed by the particles-and-fields instruments on board Voyager 1 and 2 (Blanc et al., 2002).

One important feature of Saturn's magnetosphere is its near-perfect axisymmetry, at least if we look at the magnetic field only: the dipole axis of the magnetic field is nearly aligned to the rotation axis. Thus, as we will see, one of the biggest surprises of the studies of Saturn's magnetosphere is that nearly all magnetospheric parameters display a strong rotational modulation. The discovery and understanding of this rotational modulation is, still today, one of the greatest challenges of magnetospheric science at Saturn (Carbary and Mitchell, 2013).

The size of the magnetosphere extends from 20 to 35 RS on the dayside, depending on solar wind pressure upstream. The general shape of the magnetosphere is somewhat "stretched-dipole" like, similar to the Earth's. Inside the magnetosphere, Voyager was able to identify several plasma regimes. In particular:

- the inner plasma torus, a region of cold plasma located from the rings to about 8 Rs, which Cassini showed to be populated mainly by water-related ions; this region is in near-rigid corotation.

- the extended plasmasheet, a region of warm plasma extending out of the inner torus to about 15 Rs, populated by a more rarefied and hotter population of ions and electrons.

Beyond these populations, we find the classical boundary regions on the dayside (the region close to the magnetopause) and the different regions of the magnetotail on the nightside, with the 
extended plasmasheet and tail lobes, which have been visited extensively by Cassini (André et al., 2008; Arridge et al., 2011).

Overall, the magnetic field configuration of Saturn is rather Earth-like, but the plasma populations show the dominance of a source in orbit around Saturn, which happens to be Enceladus and to a lesser extent the ring system, as we shall see later.

The plasma flow regime seems to be close to the Jovian case. The inner torus is in near-rigid corotation, and this corotation is enforced by a system of field-aligned currents, which closes at the two ends of the field line, in the thermosphere/ionosphere and in the region of the equatorial plane. This current loop transports angular momentum outwards, from the thermosphere to the plasma torus, enforcing corotation in the inner part of the equatorial magnetosphere. However, beyond a certain distance (in the range of $10 \mathrm{Rs}$ ), the effect of corotation enforcement currents becomes insufficient, and a lag of the azimuthal plasma flow behind corotation progressively develops.

In this chapter, we are going to explore the different source regions of Saturn's magnetosphere, and the associated dynamical phenomena. Once all sources are visited, we will wrap-up our exploration by summarizing the relative intensities of the different sources, and by placing the different sources in the context of a global description of Saturn's magnetosphere seen as an integrated system.

\section{Enceladus: the primary source of heavy particles in Saturn's Magnetosphere}

\subsection{The primary Enceladus source.}

Unlike Jupiter where plasma dominates the magnetosphere, Saturn's magnetosphere is dominated by neutral particles by 1 to $>2$ orders of magnitude over charged particles. These magnetospheric particles originate from many sources including Saturn's atmosphere, rings and moons (Figure 1). The dense atmosphere of Saturn's largest moon, Titan, was originally thought to be the primary source of these magnetospheric particles (Eviatar et al.. 1984), however more recent observations and data analysis indicate that the tiny icy moon, Enceladus, is actually the primary source (Dougherty et al., 2006; Porco et al., 2006). Thanks to Cassini observations, the source rates for all of these objects are much better defined. Figure 2 shows the current understanding of these source rates and illustrates that, with the exception of hydrogen, Enceladus clearly dominates over all of the other sources when producing magnetospheric particles. This surprising discovery is not yet completely understood, however it has large implications for the Saturnian system. While Enceladus has been known to be geologically active for some time, it was not expected that this tiny moon $(\sim 250 \mathrm{~km}$ radius $)$ could be such a significant part of this planetary system. Thus understanding this phenomenon has been a subject of much research and debate. 


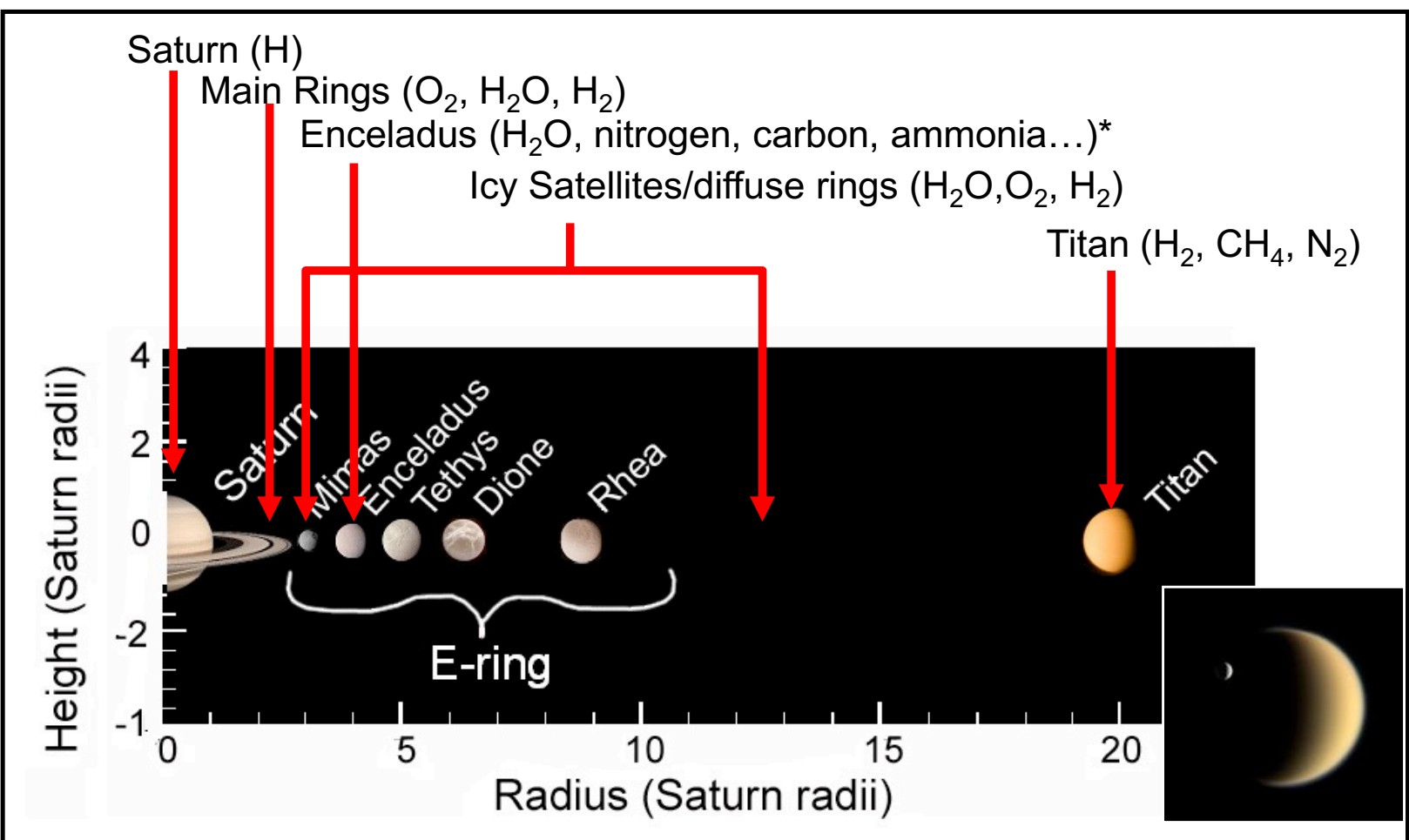

Figure 1. Graphical representation of particle sources in Saturn's magnetosphere (not to scale). (from Smith et al. 2012)

With the dramatic increase in observations as a result of the Cassini mission, several studies were undertaken to better understand this primary source of magnetospheric particles. In particular, Smith et al. (2010), Tensihev et al. (2010) Dong et al. (2011) and Hansen et al. (2011) studied the Enceladus plumes using models and/or data analysis with relatively similar results. However, Smith et al. (2010) showed noticeable levels of plume source variability, which now appears to be consistent with the recent Cassini dust observations reported by Hedman et al. (2013). Therefore, we present the Smith et al. (2010) results in more detail here. 


\section{Saturn:}
H $\quad 300^{*} \times 10^{28}(\sim 5000 \mathrm{~kg}) / \mathrm{s}$
Shemansky et al. 2009 (rough upper limit)
(Tseng et al. 2013 estimates $\sim 20 \%$ of this value)

Rings:
$\mathrm{O}_{2} \quad 0.2 \times 10^{28}(\sim 100 \mathrm{~kg}) / \mathrm{s}$
Tseng et al. 2012 (/100 at equinox)
$\mathrm{H}_{2} \quad 0.4 \times 10^{28}(\sim 13 \mathrm{~kg}) / \mathrm{s}$
Tseng et al. 2012 (/100 at equinox)

Enceladus:

$\underline{\mathrm{H}}_{2} \mathrm{O} \quad 0.3-2.5 \times 10^{28}(\sim 100-760 \mathrm{~kg}) / \mathrm{s}$ Hansen et al. 2006; Burger et al. 2007, Tian et al. 2007, Smith et al. 2010, Tenishev et al. 2010, Dong et al. 2010

$\left\{\mathrm{N}\left(\mathrm{C}^{*}\right)\right.$ 0.01-0.1 $\times 10^{28}(\sim 4-25 \mathrm{~kg}) / \mathrm{s} \quad$ Waite et al. 2006; Smith et al. 2007

Titan
$\mathrm{H}_{2}$
$0.8 \times 10^{28}$
$(\sim 27 \mathrm{~kg}) / \mathrm{s}$
Cui et al 2008
$\mathrm{N}_{2} \quad 0.01-0.02 \times 10^{28}(\sim 5-10 \mathrm{~kg}) / \mathrm{s}$
DeLaHaye et al. 2007
$\mathrm{CH}_{4} 0.01 \times 10^{28}(\sim 3 \mathrm{~kg}) / \mathrm{s}$
DeLaHaye et al. 2007
$\left\{\mathrm{CH}_{4} 0.2 \times 10^{28} \quad(\sim 55 \mathrm{~kg}) / \mathrm{s}\right.$ ?
Yelle et al. 2009; Strobel 2009\}

${ }^{*}$ Global detection (MIMI) of energetic Carbon ions $1.3 \%$ relative to water group (Mauk et al. 2009)

Figure 2. Summary of current estimated Saturn magnetospheric source rate values with references. (from Smith et al. 2012)

Smith et al. (2010) used a 3-D Monte Carlo particle-tracking, multi-species computational model (Smith et al., 2004, Smith, 2006, Smith et al., 2007 and Smith et al., 2008) to analyze the Enceladus plumes. This validated model accounts for all gravitational effects of the planet and major satellites, as well as simulating particle interaction processes including electron impact ionization and dissociation, photo-ionization and photo-dissociation, recombination, charge exchange, neutral-neutral collisions, collision with the planet, satellites and the main rings as well as escape from the magnetosphere. They used Cassini Ion Neutral Mass Spectrometer (INMS) observations of neutral water particles during the E2, E3 and E5 Enceladus encounters to help constrain key plume parameters. By conducting a parametric set of simulation runs and extracting particle densities along each encounter trajectory, they determined that the data is best fit with plume velocities of $\sim 720 \mathrm{~m} / \mathrm{s}$ and plume widths of $\sim 30$ degrees. Figure 3 shows how these parameters allow the model results to coincide well with the observations.
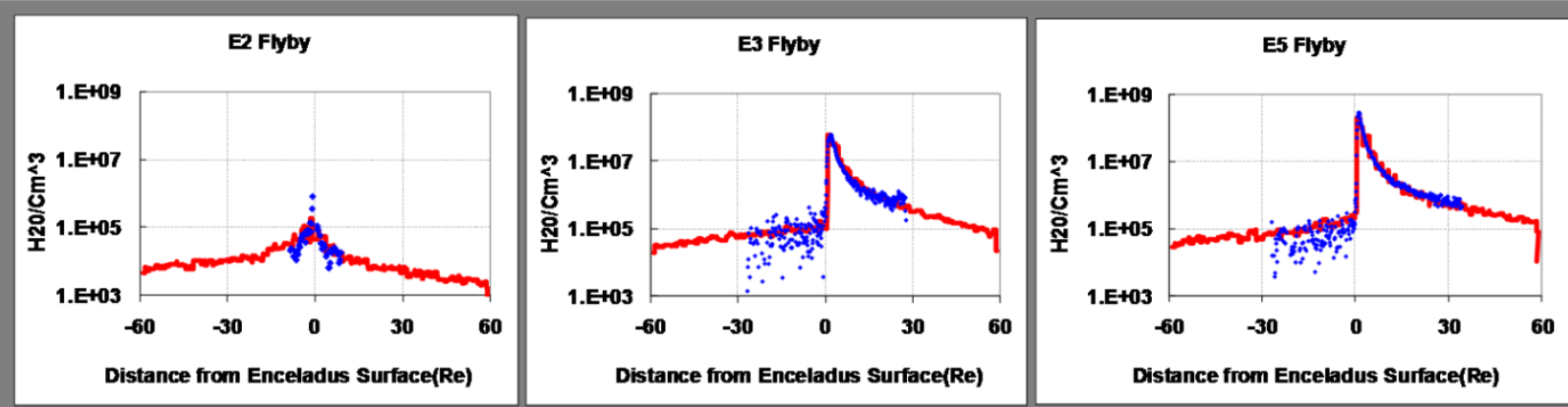
Figure 3. Comparison of best fit model results (red line: $30^{\circ}$ and 1.8 velocity ratio) with INMS measured neutral water densities (blue circles) for the E2 (left panel), E3 (middle panel) and E5 (right panel) Enceladus encounters. Results are displayed in water density $\left(\mathrm{H}_{2} \mathrm{O} / \mathrm{cm}^{3}\right)$ as a function of distance from Enceladus (in Enceladus radii or $252 \mathrm{~km}$ ) with negative values for ingress and positive for egress. Source rates for each case are adjusted so model peak densities match peak INMS densities. (from Smith et al. 2010)

Interestingly, Smith et al. (2010) were able to determine different source rates for each encounter. More specifically, they reported the following flowing source rates: E2 $\sim 2.4 \times 10^{27}$ $\mathrm{H}_{2} \mathrm{O} / \mathrm{sec}$; E3 $\sim 6.3 \times 10^{27} \mathrm{H}_{2} \mathrm{O} / \mathrm{sec}$; E5 $\sim 25.0 \times 10^{27} \mathrm{H}_{2} \mathrm{O} / \mathrm{sec}$; (E7 tentatively $\sim 9.5 \times 10^{27}$ $\mathrm{H}_{2} \mathrm{O} / \mathrm{sec}$ ). They report a factor of 3-4 variability between the E3 \& E5 encounters (the E2 trajectory only passes through the outer edge of the plumes and thus they were not as confident in that source rate). Figure 4 shows the resulting 3-D plume density distribution for the E3 trajectory.

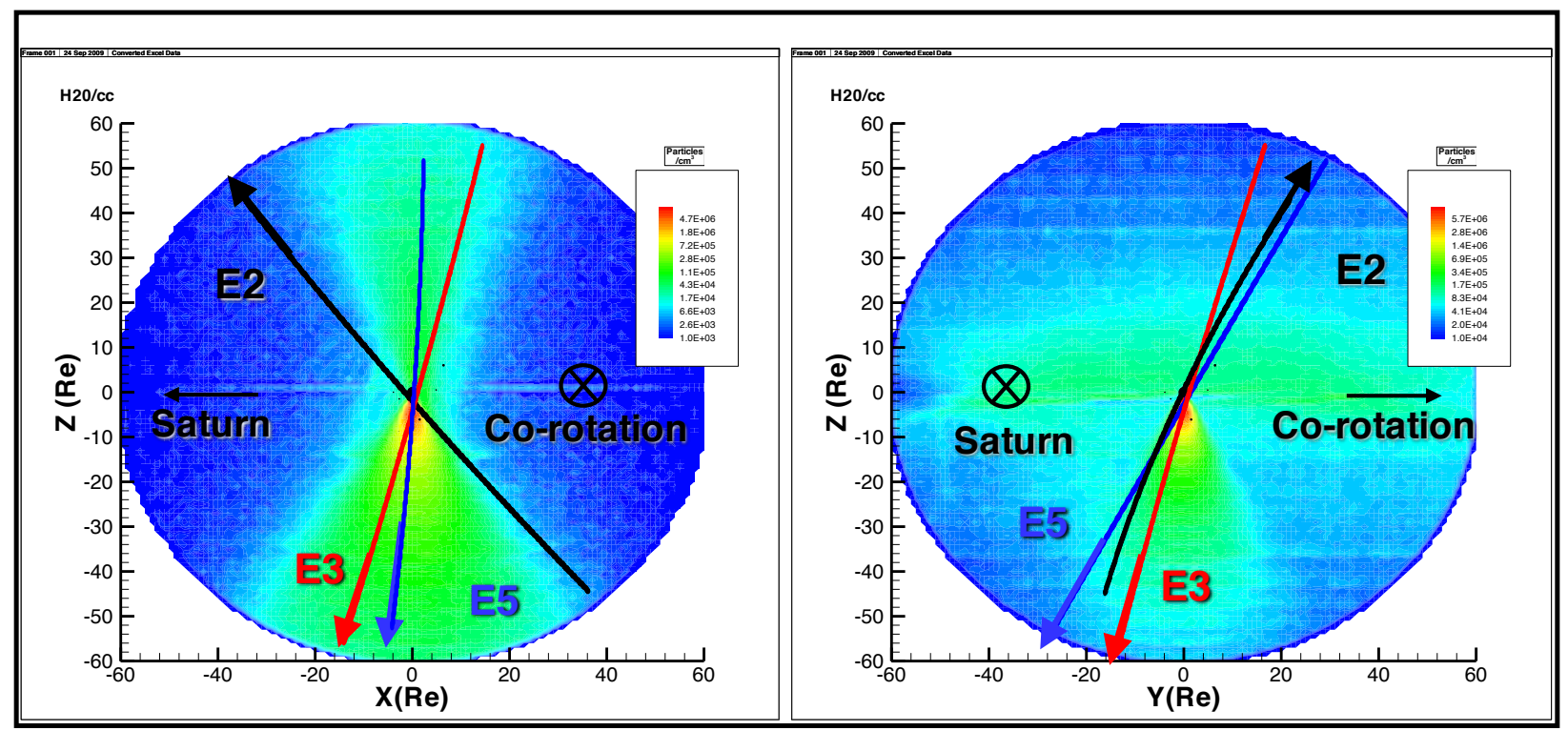

Figure 4. Contour plots of $\mathrm{H}_{2} \mathrm{O}$ density model results for the best fit Enceladus plume with $2 \mathrm{x}$ thermal velocity and ejection angle constrained to $+/-30^{\circ}$ for the E3 source rate (divide densities by $\sim 15$ for E2 and multiply by $\sim 4$ for E5). Plots show $Z$ axis (in Enceladus radii, or Re) based on the rotational axis, $\mathrm{X}$ axis (in Re: -X toward Saturn) in panel (a) and $\mathrm{Y}$ axis (in Re: +Y in corotational direction) in panel (b). E2 (red), E3 (blue) and E5 (black) trajectories shown in each panel. (from Smith et al. 2010)

As mentioned above, several other plume characterization studies were also conducted. In particular, Saur et al. (2008) constrained a neutral atmospheric model with Cassini magnetometer observations to report variable source rates of $\sim 1600 \mathrm{~kg} / \mathrm{s}$ for the E0 encounter and $\sim 200 \mathrm{~kg} / \mathrm{s}$ for the E1 and E2 encounters. Tenishev et al. (2010) applied a test particle model constrained by Cassini neutral particle (INMS) observations (with 8 variable plume sources to examine relative strength) to simultaneously fit the E3 and E5 encounters. They report source rates within a factor of 2 (E5 highest). Also, Dong et al. (2011) used an analytic model constrained by the same INMS observations of the E3, E5 \& E7 encounters to determine source rates ranging from $\sim 1.5$ to $\sim 3.5 \times 10^{28} \mathrm{H}_{2} \mathrm{O} / \mathrm{sec}$ (factor of 2). However, Hansen et al. (2011) reported less than $20 \%$ plume activity variability based on Cassini Ultraviolet Imaging 
Spectrograph (UVIS) observations of plume occultations in 2005, 2007 and 2010. Thus, the amount (if any) of plume variability was debated for several years and the limited number of observations made it difficult to resolve these inconsistent results.

More recent observations appear to support plume variability. Hedman et al. (2013) studied the relative brightness of dust particle observations in 252 Cassini Visual and Infrared Mapping Spectrometer (VIMS) images. Although these results involve dust particles, they provide a good proxy for relative vapor source rate. Interestingly, they find a factor of three variability in the plume source rate. Additionally, they find that this source rate is a function of the Enceladus mean anomaly with the highest source rates occuring when the satellite is furthest from Saturn, which supports the theory of enhanced source strength when tiger stripes are under tension. Figure 5 shows their results organized by orbital phase. This figure also shows that the Smith et al. (2010) plume variability results coincide well with the Hedman et al. (2013) results if one assumes orbit symmetry.

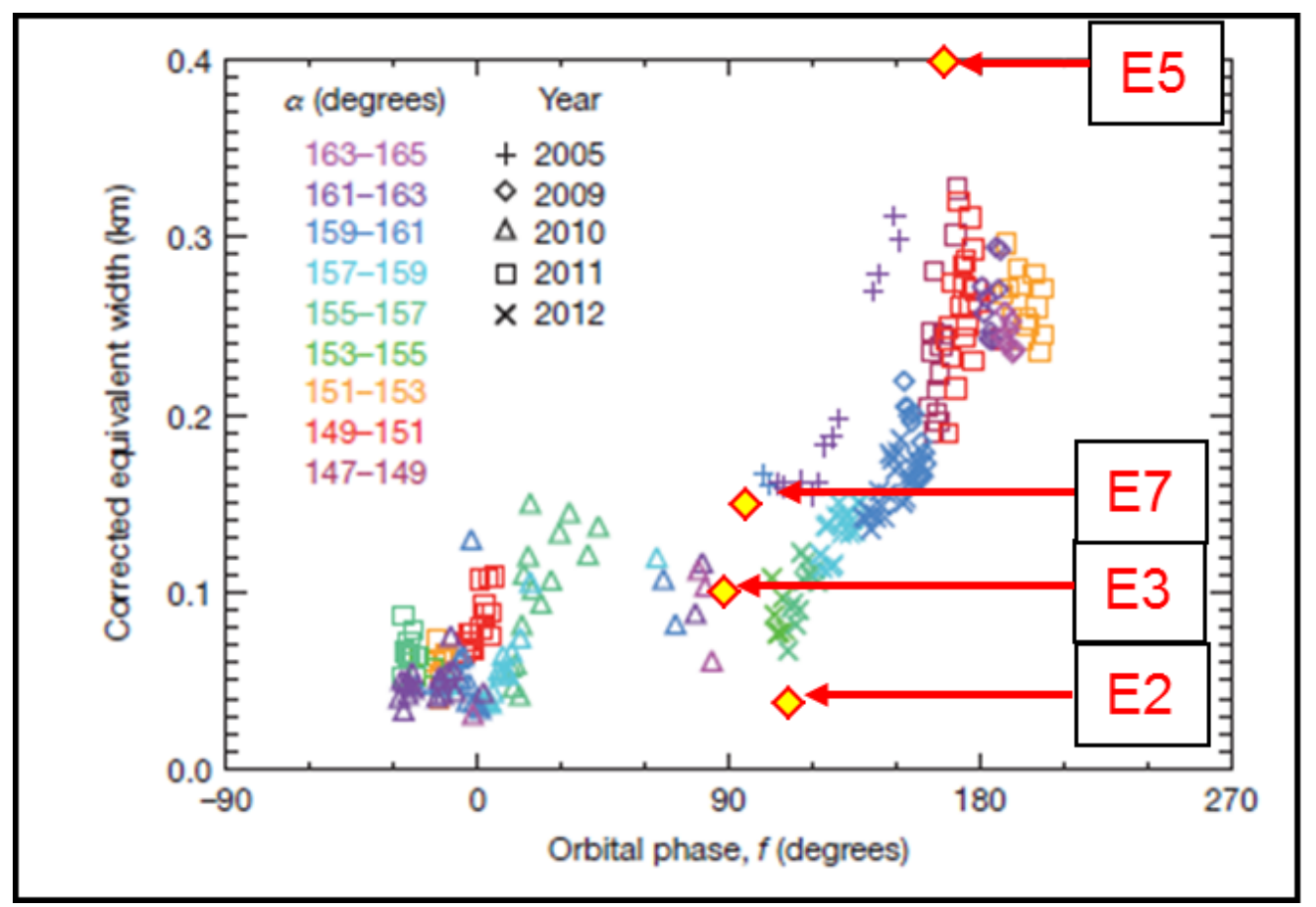

Figure 5. Figure from Hedman et al. (2013) showing plume activity as a function of Enceladus mean anomaly (width vs. orbital phase). Smith et al. (2010) scaled source rate values for the E2, E3, E5 and E7 encounters are also plotted (assuming orbital symmetry).

Therefore, our understanding of Enceladus as the primary source of heavy particles in Saturn's magnetosphere has noticeably increased. However, one must remember that these are neutral particles, which do not necessarily directly translate to a plasma source rate near this moon. The region near Enceladus is very complex and remains a topic of much debate. This is a region consisting of relatively large neutral particle densities, plasma, negative ions and dust grains where the plasma co-rotational $(\sim 36 \mathrm{~km} / \mathrm{s})$ speed is not much faster than the Keplerian orbital 
speeds $(\sim 12.5 \mathrm{~km} / \mathrm{s})$. In terms of plasma mass loading, photoionization lifetimes are very long (on the order of years) and the electron-impact ionization rates dramatically decrease in Saturn's inner magnetosphere because the core electron population is below ionization cut-off energies. Thus, the region near Enceladus is not well suited for ion production. Tokar et al. (2009) report a "plasma stagnation region" within a couple of satellite radii of Enceladus (Figure 6) where the co-rotational plasma flow decreases significantly because of ion momentum loading (mostly through charge exchange). This should cause increased ionization, however Coates et al. (2013) report less than an order of magnitude ionization increase in this region. Additionally, this slower region should also dramatically increase the charge exchange rates, which would impede the process of creating new ions. Fleshman et al. (2010) use modeling to report that neutral particles should dominate over charged particles by a ratio of 40:1 near Enceladus and most of the electron impact ionization occurs from the higher energy electrons which should make up $<1 \%$ of the total electron population. Thus, while Enceladus is a major source of neutral particles, the resulting charged particles are more likely to be produced further away from the moon.

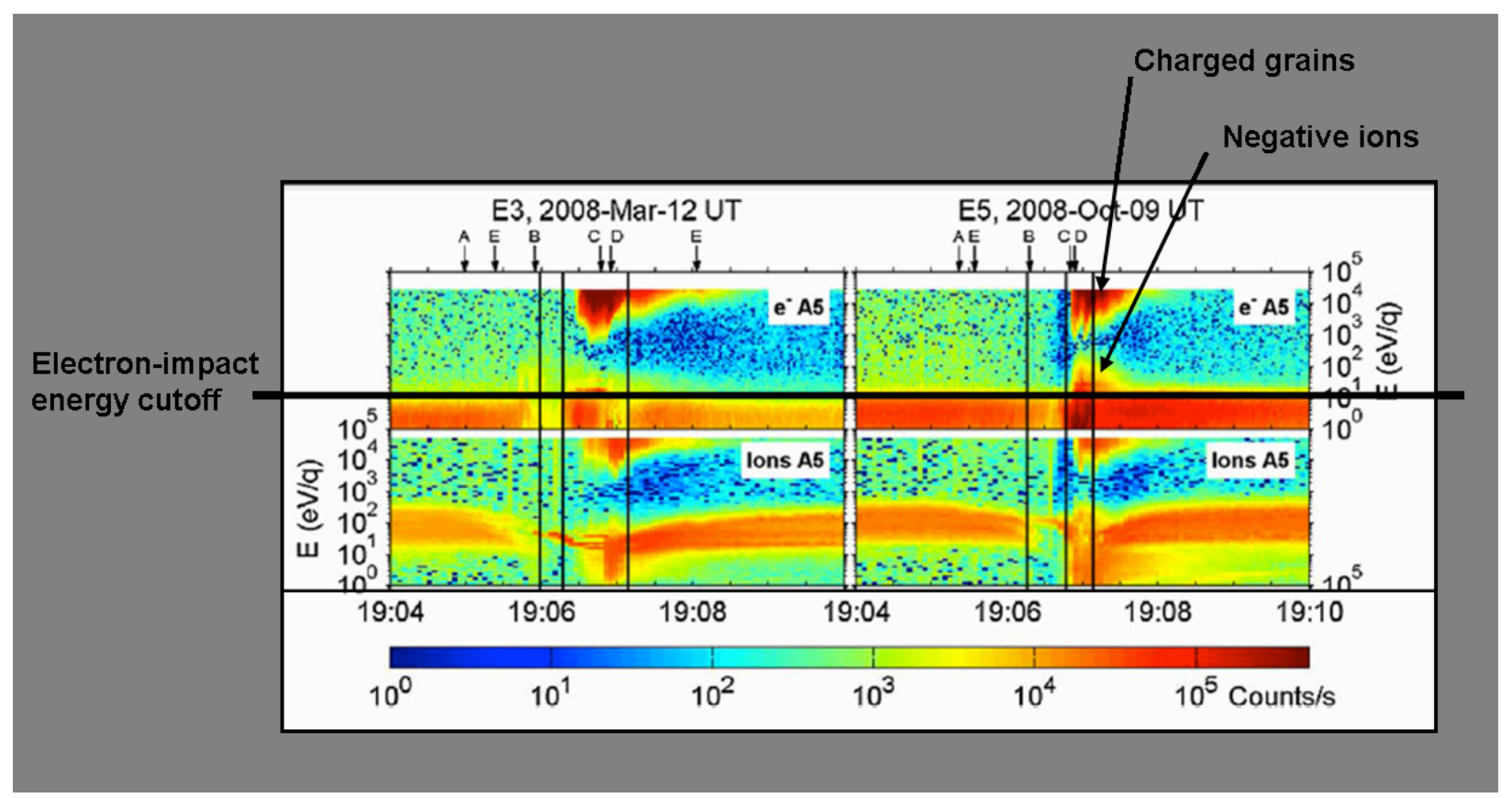

Figure 6. Annotated plot from Tokar et al. (2009) showing Cassini Ion mass spectrometer and electron spectrometer spectra for the E3 and E5 flybys. Data is counts as a function of time (UCT) and energy $(\mathrm{eV})$. The ionization cut-off energy is plotted on the electron spectra and the charged grain and negative ion signals are also annotated.

\subsection{Resulting extended sources}

As mentioned above, Enceladus actually serves indirectly as a much more extended source of particles in Saturn's magnetosphere. This extended source is moderated by source particle interactions with electrons, photons, ions and other neutral particles. More specifically: 
- Electron-impact interactions can cause direct ionization as well as dissociation into daughter neutral and/or ionized species. In the case of dissociation, surviving neutral particles also have a small energy increase, which tends to spread out the neutral clouds.

- The neutral particles can also interact with solar UV photons, which interact similarly to the electron-impact processes. However these rates vary with solar activity.

- Neutral particles can interact with ions either through a simple collision or one where charge exchange occurs (effectively ionizing the neutral and neutralizing the ion). This process is much more efficient as plasma and neutral particle velocities approach each other. The net result of charge exchange is that while a fresh ion is created, an ion has become an energetic neutral particle so there is essentially no net increase in the ion population. Thus this process is not considered as plasma mass loading, but only as plasma momentum loading (i.e. the plasma flow tends to slow down as this fresh ion is picked up). This process dramatically spreads out the neutral clouds. Fleshman et al. (2012) indicate that gyrophase-dependant collisions have more of an impact on $\mathrm{OH}$ local densities than on the other water group species.

- Some neutral particles can also interact with other neutral particles in the densest regions. This is a result of enhanced dipole interactions (Farmer, 2009, Cassidy and Johnson, 2010) and tends to spread out the neutral particles even more.

Figure 7 shows relative contributions of each of the processes, which cause a spread in the neutral oxygen torus.

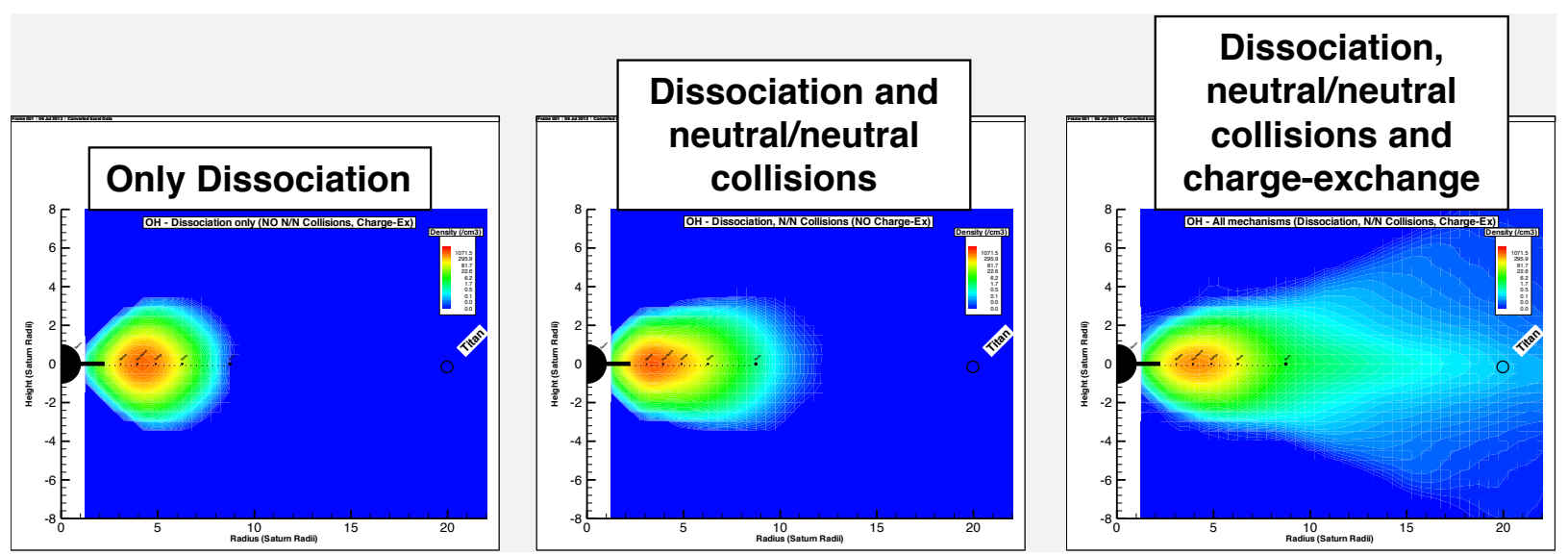

Figure 7. Model results showing neutral oxygen distribution originating from the Enceladus plumes for differing theoretical cases: with only dissociation (left), with dissociation and neutral-neutral collisions present (middle) and with dissociation, neutral-neutral collisions and charge-exchange active (right). Data is oxygen density $\left(\mathrm{cm}^{-3}\right)$ in the R-Z plane (in Saturn radii) with Saturn on the left of each panel. (from Smith et al. 2010)

These processes interact in a complex manner with interaction lifetimes as the key driver in the dynamics of these distributed sources. Figure 8 shows the interaction lifetimes in Saturn's magnetosphere for water and hydrogen as a function of radial distance from Saturn (with 
Enceladus at $\sim 4 \mathrm{Rs}$ ). This figure helps to illustrate how photolysis is constant throughout all radial distances while electron-impact is most significant (and dominant) at 7-9 Rs where the electron temperature and density is most conducive to interaction. This figure also shows the charge exchange interaction rate dramatically increasing in the inner magnetosphere. These lifetimes combine to create and accelerate an extended source of particles with only $\sim 11 \%-26 \%$ of the original Enceladus particles actually being ionized, $\sim 23 \%-43 \%$ lost through collision (with Saturn, the moons and rings) and $\sim 31 \%-66 \%$ escaping the magnetosphere as a result of charge exchange (Cassidy and Johnson, 2010, Fleshman et al., 2010).

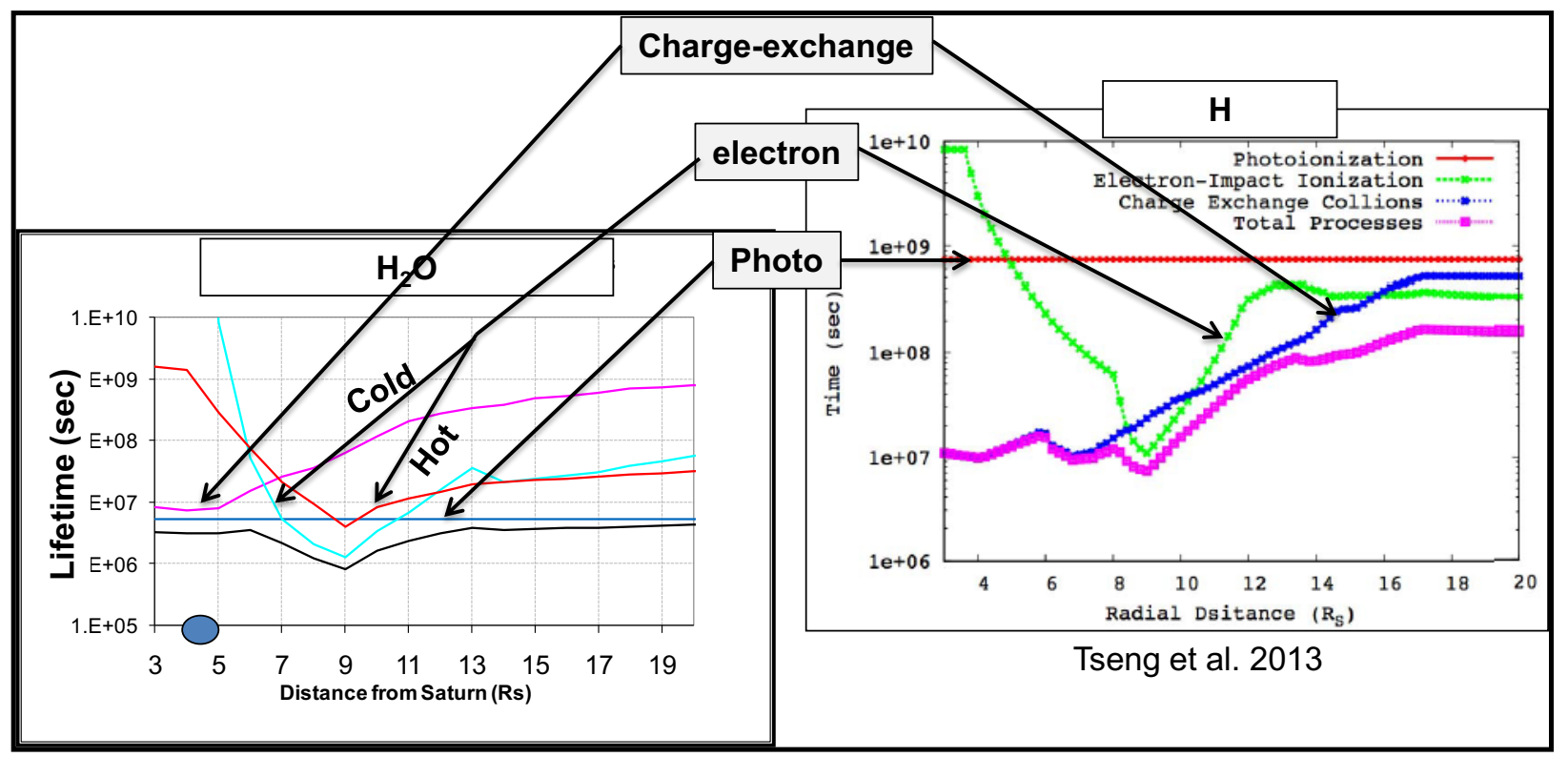

Figure 8. Interaction lifetimes (seconds) for $\mathrm{H}_{2} \mathrm{O}$ (left panel) and $\mathrm{H}$ (right panel) as a function of radial distance from Saturn (in Saturn radii). Charge-exchange, electron-impact and photolysis processes are annotated with the right panel offset so the values on the $\mathrm{H}$ lifetime $\mathrm{y}$-axis are aligned with the $\mathrm{H}_{2} \mathrm{O}$ lifetime values. (from Smith et al. 2012)

The result is a series of co-orbiting neutral particles that form multiple Enceladus-generated neutral tori, which serve as a distributed source of plasma in the magnetosphere. Figure 9 shows the timescale and process that lead to tori formation. The Enceladus plumes provide the original water source species, which escape with relatively low velocity. The result is a relative confined torus of $\mathrm{H}_{2} \mathrm{O}$ particles near Enceladus' orbit (this feature is expanded through neutral-neutral interactions). On the order of about 2.5 months, $\mathrm{OH}$ molecules are created through dissociation (primarily photo) creating a more extended torus. This torus is expanded via charge-exchange, neutral-neutral interactions as well as energy obtained from dissociation. Next, an even more extended $\mathrm{O}$ torus is created (on the order of $\sim 1.5$ months) that is spread out through the same mechanisms. Finally, dissociated hydrogen from these interactions (as well as from Saturn and Titan) forms a much more extended neutral torus. Thus Enceladus indirectly generates multiple distributed magnetospheric neutral sources. These sources in turn serve as plasma sources. As mentioned above, ions are much more likely to be produced in the 7-9 Rs range which coincides with the peak in Saturn's electron and ion flux tube content (Sittler et al., 2008, Chen et al., 2010) as shown in Figure 11. It is also important to consider that the heavy ions are fairly 
constrained to the equatorial plane while lighter ions are much more extended and peak densities actually occur off of the equatorial plane. Finally, plasma transport dominates in the middle and outer magnetosphere, while ions in the inner magnetosphere tend to be lost through recombination (Figure 12). Bagenal and Delamere (2011) also show that local sources/losses dominate over radial transport at distances closer than 8 Rs of Saturn. They also show that the ionization rate does not keep up with the neutral source rate variations.

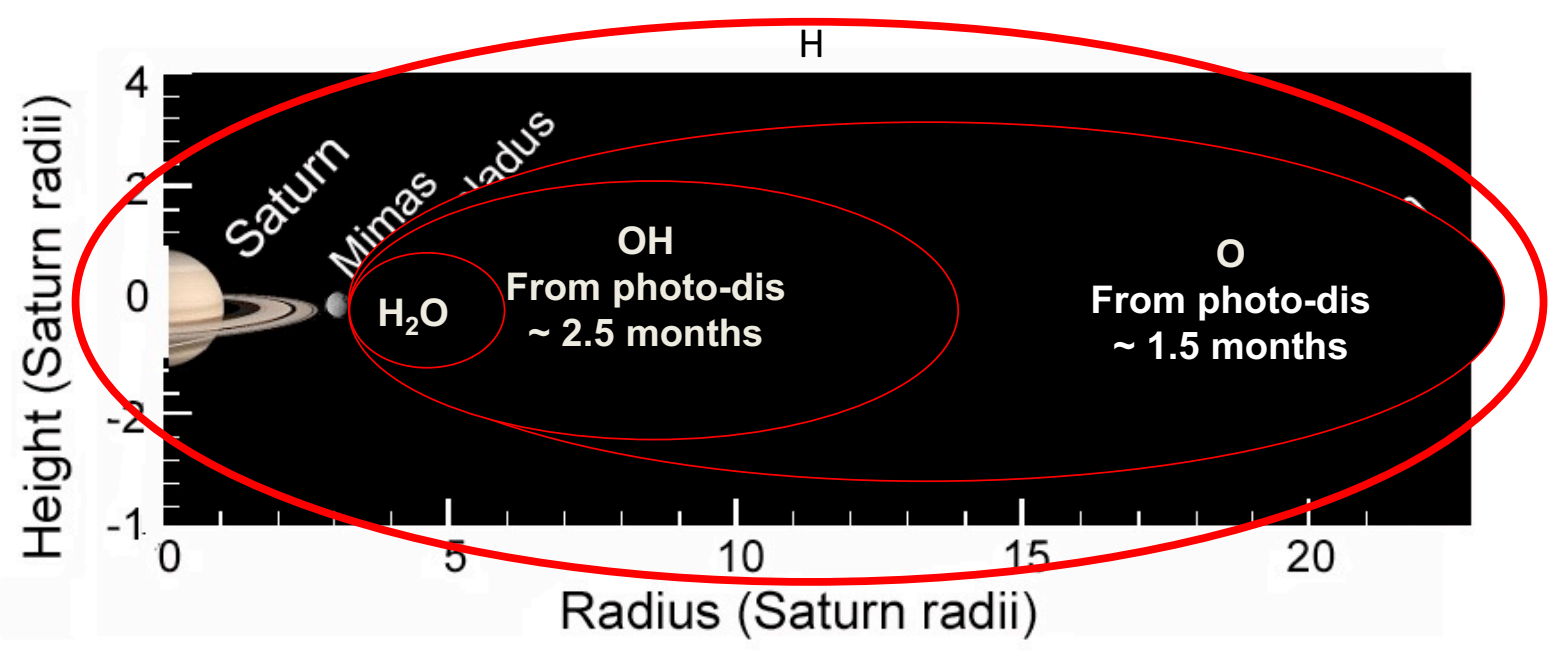

Figure 9. Graphical representation of the rough distribution of resulting neutral $\mathrm{H}_{2} \mathrm{O}, \mathrm{OH}, \mathrm{O}$ and $\mathrm{H}$ tori with the approximate times required to produce these species from the original water source. (from Smith et al. 2012) 


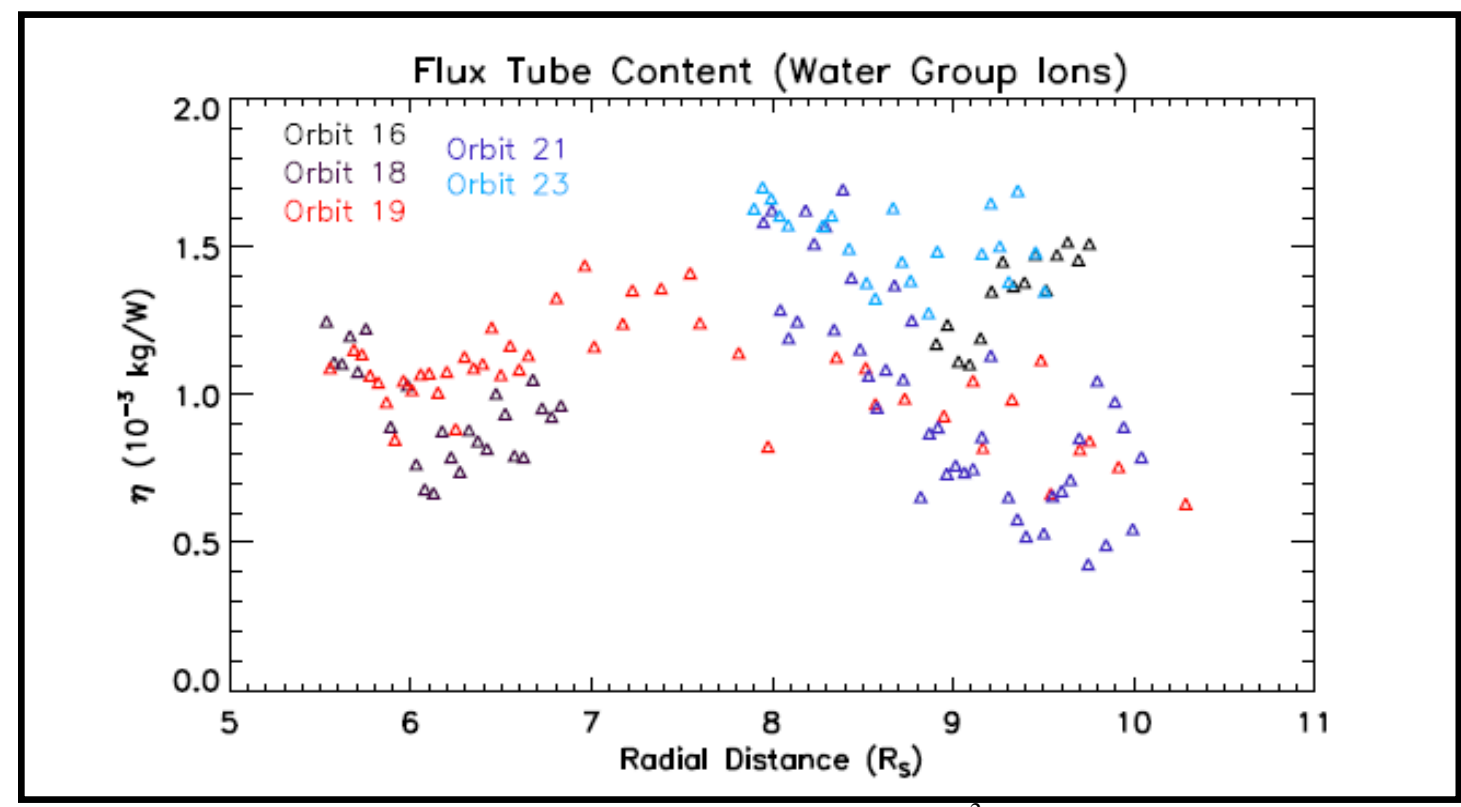

Figure 10. Water group ion peak flux tube content $\left(10^{-3} \mathrm{~kg} / \mathrm{W}\right)$ as a function of radial distance from Saturn (from Chen et al. 2010).

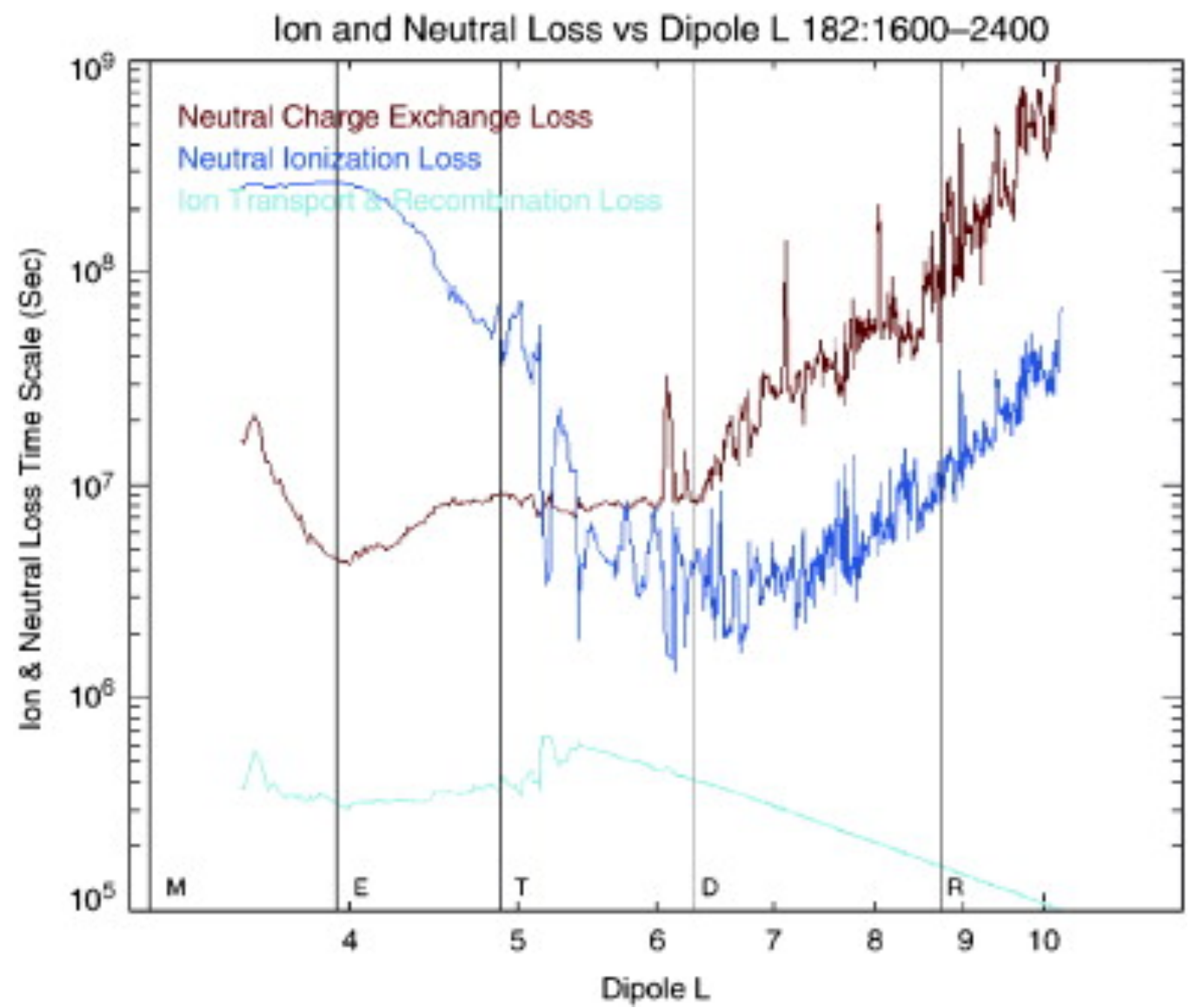


Figure 11. Comparative lifetimes (seconds) for charge exchange (brown), neutral loss to ionization (blue) and combined ion loss to recombination and transport (light green) (from Sittler et al., 2008).

\section{Dusty plasmas in Saturn's plasma torus}

The water vapors and ice grains expelled from Enceladus' south pole are one of Cassini's most exciting discoveries [Dougherty et al., 2006; Porco et al., 2006; Waite et al., 2006]. Furthermore the state of the plume and its surrounding plasma constitute a so called "dusty plasma" environment. In this section we describe briefly what is a dusty plasma, how the observation has been interpreted, and its possible consequences for the magnetosphere.

Dusty plasmas are plasmas containing charged particles (dust). The particle sizes are typically nanometers to micrometers and they are massive compared to the plasma elementary charged particles. The dust charge can be negative or positive and the grain charge number varies from single to several thousands. As a result the charged particles in dusty plasmas are under the control of both gravity as well as electro-magnetic forces.

In addition to this the charged dust particles are strongly coupled to the surrounding plasmas depending on the characteristic lengths. When the inter grain distance $(a)$ is larger than the plasma Debye length $\left(\lambda_{D}\right)$, the situation $r_{d} \ll \lambda_{D}<a$ holds, where $r_{d}$ is the grain radius, the charged particles are considered as isolated among the plasma. This situation is referred to 'a dust-in-plasma'. A 'real' dusty plasma occurs when $\mathrm{r}_{\mathrm{d}} \ll a<\lambda_{\mathrm{D}}$, wherein charged dust particles participate in the collective behavior [e.g., Shukla and Mamun, 2002].

The dust and plasma conditions found near Enceladus are in the dusty plasma state. This has been suggested by the Langmuir probe that measures the electron and ion densities of cold plasmas (Figure 12). The observed large ion densities $\left(30,000-100,000 \mathrm{~cm}^{-}{ }^{3}\right.$ ) and low electron densities (2000-4000 $\mathrm{cm}^{-3}$ ) in the plume region must be the result of the attachment of electrons onto the dust particles [e.g., Waite et al., 2006]. Incidentally, the ion density obtained here exceeds the expected amount from just photo ionization (see Section 2 for ionization), indicating that an additional ionization mechanism is needed to generate this large amount of plasma. The electron densities are generally more than an order of magnitude smaller than the ion densities, as if more than $90 \%$ of the electrons were missing from the region. This electron density dropout is due to the electron attachment to the dust grains [Farrell et al., 2009]. It has been confirmed that the micrometer sized negatively charged dust observed by the Cosmic Dust Analyser [CDA] (Kempf et al., 2008) as well as the Radio and Plasma Wave Science (RPWS) signals [Kurth et al., 2006; Farrell et al., 2009] coincide within this region. Also both positive and negative nanometer sized small grains have been observed by the plasma particle detectors (Jones et al., 2009; Hill et al., 2012), which support the conclusion that the majority of the dust particles are negatively charged (Figure 13). Since the electric charges of the grains are proportional to the grain surface, large micrometer grains can hold several thousand charges while nanometer grains are often singly charged (e.g., Horányi, 1996; Yaroshenko et al., 2009].

Comparing the electron and ion density differences obtained from the Langmuir probe and the dust size distributions from CDA as well as CAPS (CAssini Plasma Spectrometer), a large amount of sub-micron grains are inferred to exist as the majority of the negative charge carriers 
(Figure 14). Using the electron/ion density differences, the average grain potential, and the modeled dust size distributions, the dust density was estimated about $300 \mathrm{~cm}^{-3}$, thus the inter grain distance is about $0.13 \mathrm{~cm}$, which is smaller than the local Debye length $(\sim 6.04 \mathrm{~cm})$ [Morooka et al., 2011]. Furthermore, the estimated dust condition and plasma condition satisfy the Havnes condition for a dusty plasma [Havnes et al., 1993].

Another feature that indicates the coupling between charged dust and plasmas is that the plasma speeds around the moon are slowed down to the gravitational speeds (Farrell et al., 2010; Morooka et al., 2011). The slow plasma speed is consistent with the non-wake signature of the ion density. If the plasma co-rotates with Saturn's magnetic field the moon should be an obstacle to the ions with large gyro radius and the magnetospheric ions could not enter behind the moon. However, no such ion depletion has been observed, indicating that the ambient plasma moves at almost the same speeds as the moon Enceladus. The observed large electron density depletion in the wake region should be due to the negatively charged dust.

Dusty plasmas have been studied theoretically (e.g., Goertz and Ip, 1984; Whipple et al., 1985; Havnes et al., 1987) and verified in the laboratory (e.g., Xu et al., 1993). Except for a few direct measurements in Earth's upper atmosphere (Reid, 1990; Havnes et al., 1996), there have been no in situ observations of dust-plasma ensembles in space. Simon et al., (2012) suggested that the negatively charged dust grains in the plume act as a sink for "free" electrons and yield a reversal in the sign of the Hall conductivity, resulting in a slowdown of the ions [Kriegel et al., 2011]. It is important to note that the negatively charged dust and its effect on the plasma dynamics appear not only in the plume but also in a large region around the moon Enceladus (Wahlund et al., 2009; Farrell et al., 2012). The magnetospheric plasma speeds are confirmed to be often slower than the co-rotating speed (Wilson et al., 2009; Thomson et al., 2010; Holmberg et al., 2012), which could be associated with the negatively charged dust near the E ring (Holmberg et al., 2014). 


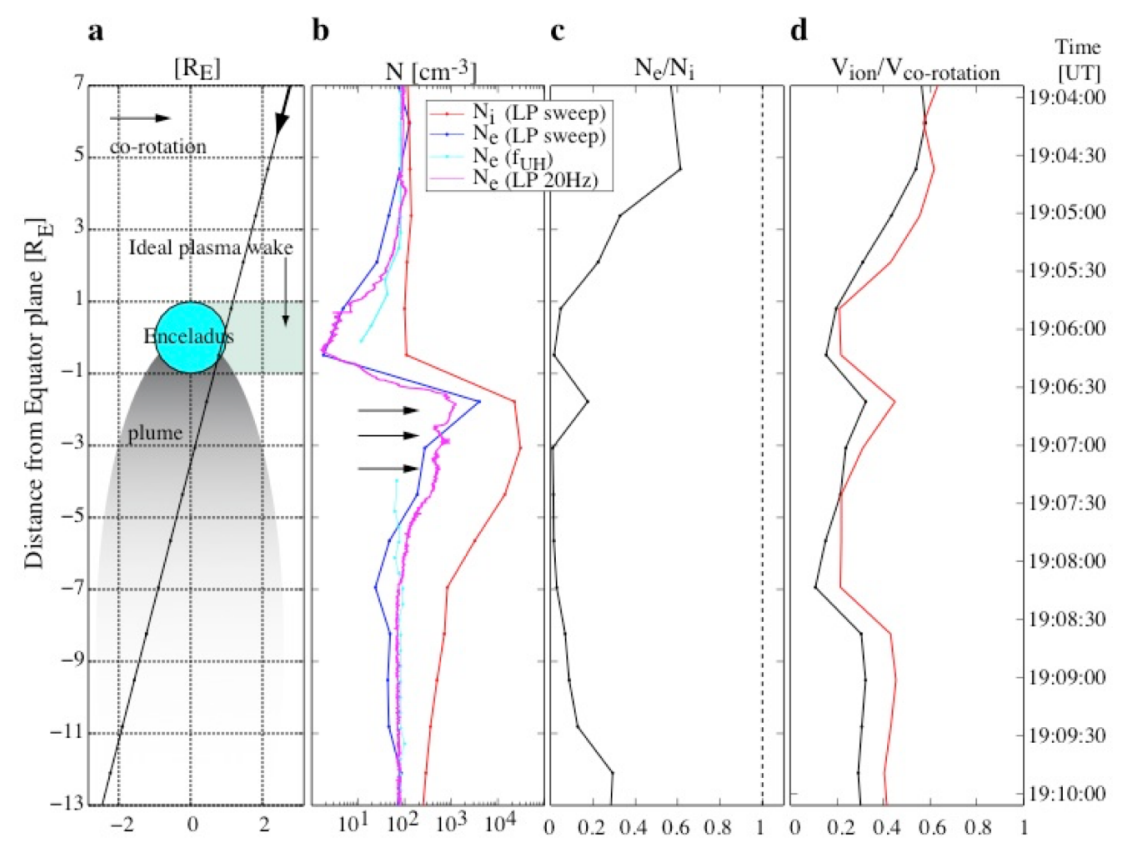

Figure 12. Cassini RPWS/LP observation from Enceladus E3 encounter. (a) Spacecraft trajectory in Enceladus frame. (b) Electron/ion densities. (c) The LP electron to ion density ratio. (d) Ion speed relative to the rigid corotation speed in the spacecraft frame of reference. See Morooka et al., [2011] for detail.

the rigid corotation speed in the spacecraft frame of reference. (from Morooka et al., 2011) 

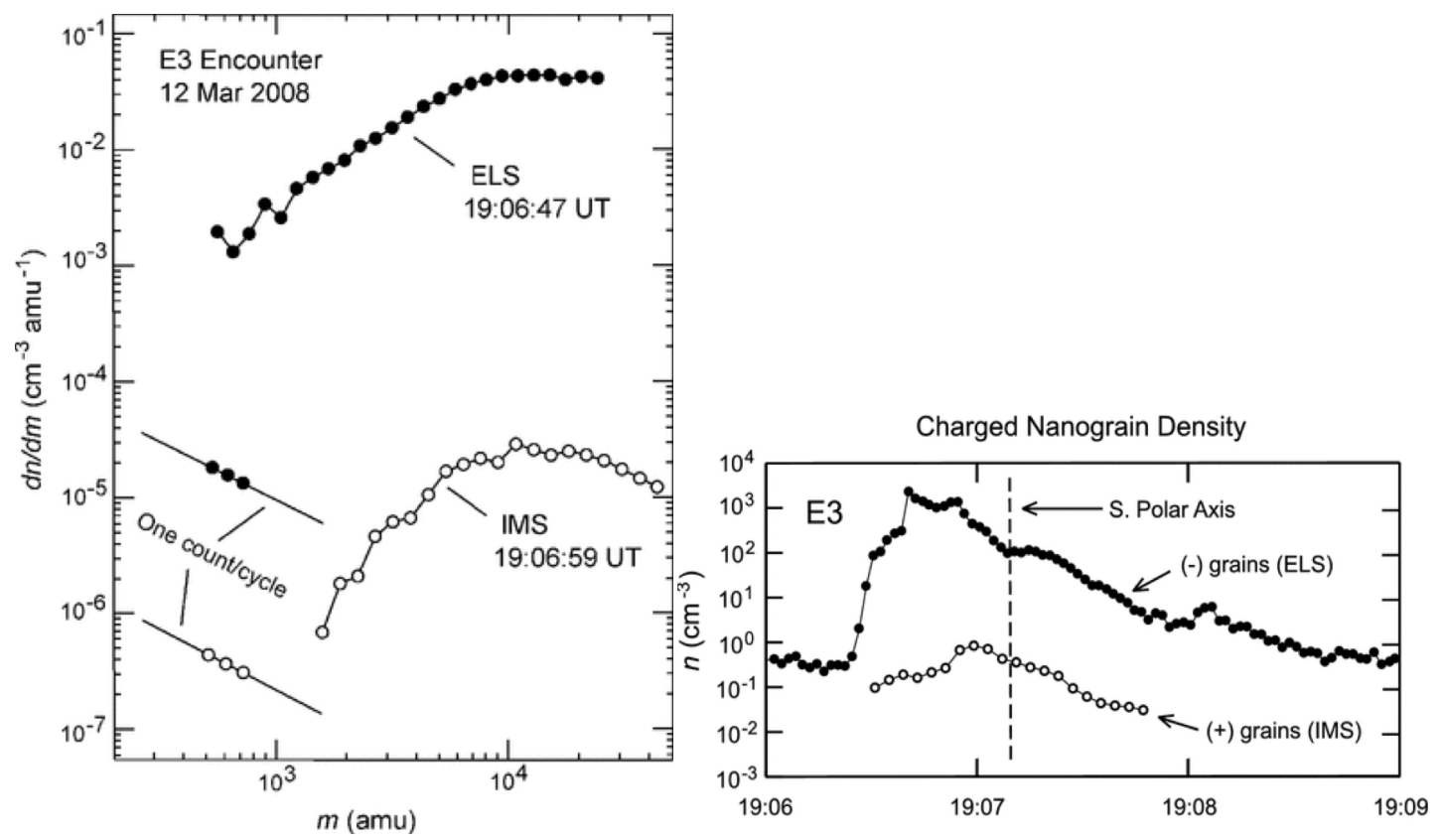

Figure 13. CAPS observations of the charged nanometer grains. Left: The mass distribution functions of the positive (white circle) and negative (black dots) charged particles. Right: Densities of the positive and negative nano-grains. [Hill et al., 2012]

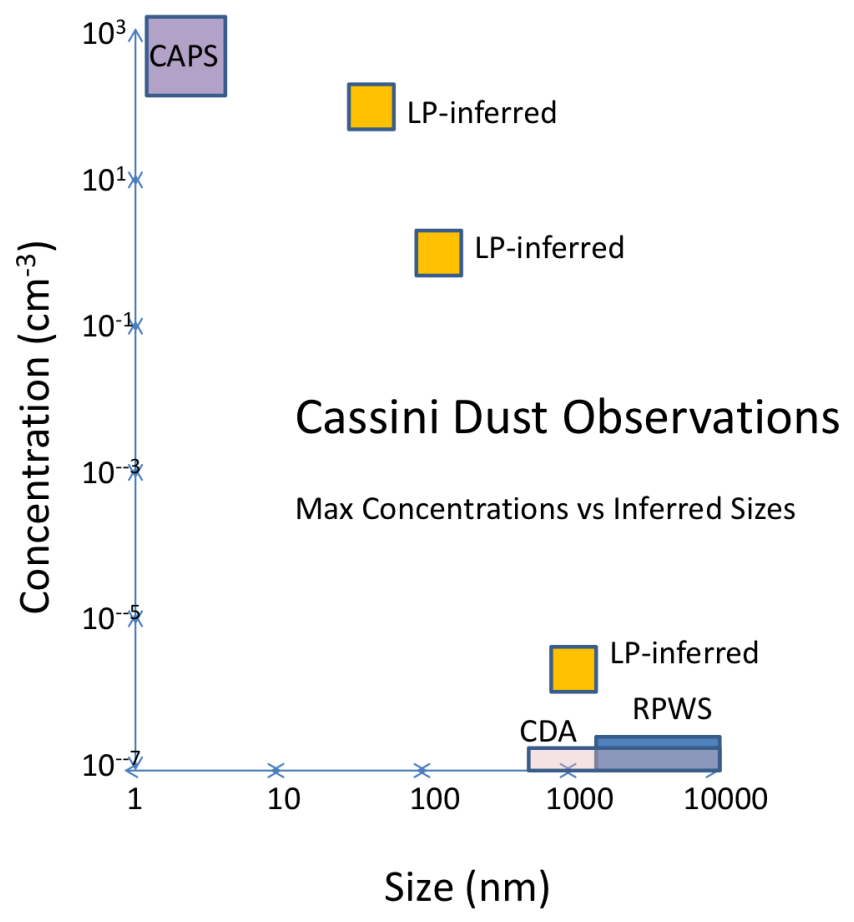

Figure 14. The schematic view dust size distribution expected in the plume. Courtesy of W. Farrell. 


\section{Titan}

Titan orbits Saturn at a distance of $20 \mathrm{R}_{\mathrm{S}}$. With its extended atmosphere composed primarily of $\mathrm{N}_{2}$ and $\mathrm{CH}_{4}$ and apparent lack of internal magnetic field, it interacts with Saturn's magnetospheric plasma and the solar wind in a cometary fashion producing an induced magnetosphere. Titan can act as a source of neutrals through atmospheric escape processes, which primarily eject $\mathrm{H}_{2}$. It can also act as a source of ionized plasma through ion outflow and pickup-like processes. Titan is also a sink of magnetospheric plasma, albeit a small one compared to the major processes that act on a global scale like charge-exchange or tail reconnection.

Prior to the Cassini exploration of the Saturn system Titan was believed to be a significant plasma source with enough protection from the solar wind due to its magnetic shielding by Saturn's magnetosphere so as to develop an observable gas torus (Smith et al., 2004). However, Cassini observations have revealed that the region near Titan was more exposed to the solar wind and magnetosheath plasma than anticipated when Titan was on the dayside (Achilleos et al., 2008) and that the neutral source was more benign than previously expected (e.g. Tucker et al.,2009; Bell et al., 2011). From magnetospheric measurements at 12:00 Saturn local time Titan is expected to be in the solar wind for $5 \%$ of the time, exposing it to enhanced solar electrons that cause greater losses of the neutrals (Achilleos et al., 2008).

Titan loses atoms and molecules from its atmosphere as ionized and neutral material. Several processes have been proposed as sources from Titan including thermal escape (Cui et al., 2008; Bell et al., 2011), chemically-induced escape (De La Haye et al., 2007), slow hydrodynamic escape (Strobel 2008, 2009; Yelle et al., 2008), pick-up ion loss and ionospheric outflow (Ledvina et al., 2005; Wahlund et al., 2005; Sillanpaa et al., 2006; Ma et al., 2006; Hartle et al., 2006; Coates et al.. 2007a; Edberg et al., 2011; Westlake et al., 2012; Coates et al., 2012) and plasma-induced atmospheric sputtering (Shematovich et al., 2003; Michael et al., 2005; De La Haye et al., 2007).

Titan's neutral source comes primarily in the form of $\mathrm{H}_{2}$, which readily escapes Titan's atmosphere (Cui et al., 2008). ENA observations of Titan's extended exosphere region have found that the $\mathrm{H}_{2}$ is found with appreciable densities radially out to the predicted Hill sphere radius of $60,000 \mathrm{~km}$ (Brandt et al., 2012). Debates within the atmospheric modeling community have argued whether $\mathrm{H}_{2}$ escape is limited by the Jeans escape rate (Bell et al., 2010, 2011) or is escaping at a significantly greater rate (Cui et al., 2008). Exospheric simulations using Direct Monte Carlo Simulation have found the $\mathrm{H}_{2}$ escape rate to be consistent with Jeans escape (Tucker et al., 2009).

Titan is also a source of $\mathrm{N}_{2}$ and $\mathrm{CH}_{4}$, though their mass is closer to the mean mass of the atmosphere and they are thus much more benign in their escape rates. $\mathrm{CH}_{4}$ and its escape rate have sparked heated debates in the modeling community as its escape rate implied from the atmospheric measurements depends on the assumptions regarding the eddy diffusion parameter in Titan's upper atmosphere and assumptions as to whether Titan is in hydrothermal equilibrium. Yelle et al. (2008) initially utilized the in-situ INMS measurements to show that either the eddy diffusion rate at Titan is relatively high and the escape rate low or vice versa. Bell et al. (2014) have recently shown that the INMS constraints provided by simultaneously fitting the altitude 
profiles of $\mathrm{N}_{2}$ (and its isotopes), $\mathrm{CH}_{4}$ (and its isotopes), $\mathrm{Ar}$, and $\mathrm{H}_{2}$ are best met by a high eddy diffusion coefficient and a small $\mathrm{CH}_{4}$ flux - a result that is reinforced by the relatively modest amount of carbon-bearing ions seen by CAPS in the near Titan environment (Smith et al., 2012). Cassini and Voyager have passed through Titan's induced magnetospheric tail region allowing for measurements of Titan source plasma being accelerated into Saturn's magnetosphere. From these measurements the mass loss rate due to pick-up ion formation and sweeping combined with ionospheric outflow is compatible with a value of roughly $10^{25} \mathrm{amu} / \mathrm{s}$ (Wahlund et al., 2005; Hartle et al., 2006; Coates et al., 2007a, 2012). The Voyager plasma instrumentation did not have sufficient energy or mass resolution to determine the composition of these ions as they left Titan other than they consisted of a heavy and a light component. This led to the assumption that the ions were classical pick-up ions produced through the local ionization of $\mathrm{CH}_{4}$ and $\mathrm{N}_{2}$ that had escaped Titan's atmosphere. Cassini observations both with the CAPS and INMS instruments have found that the composition of the ions flowing from Titan is mainly ionospheric with significant amounts of $\mathrm{CH}_{5}^{+}, \mathrm{C}_{2} \mathrm{H}_{5}^{+}$, and $\mathrm{HCNH}^{+}$, that cannot be produced in Titan's sparse exosphere (Westlake et al., 2012, Coates et al., 2012). From this, and the prevalence of "fossilized" magnetic fields in Titan's ionosphere it is clear that magnetospheric field lines penetrate deep into Titan's ionosphere where ion-neutral chemistry acts to mass load the field lines, which then carry Titan's ionospheric plasma away (Coates et al., 2012, Wellbrock et al., 2012). These ions have long lifetimes in Titan's exosphere due to the declining electron density and increasing electron temperature and can therefore remain as ions traveling downtail from Titan.

Combining these source rates one obtains a total rate that ranges between 0.03 and $0.5 \times 10^{29}$ amu/s of primarily $\mathrm{H}_{2}$ and lesser amounts of $\mathrm{N}_{2}$ and $\mathrm{CH}_{4}$ along with various compositions derived from these neutrals (Johnson et al., 2010). This source rate is significantly less than the Enceladus and rings source rates, and given Titan's proximity to the edge of the magnetosphere it is clear that this material is readily picked up into the solar wind and is not a major source of Saturn's magnetosphere.

\section{The solar wind}

In order to understand the influence of the solar wind, and its potential role as a plasma source for Saturn's magnetosphere, it is critical to study the large-scale structure upstream of Saturn to understand its impacts upon Saturn's magnetopause. The model of Parker (1958) predicted that as the solar wind evolves throughout the heliosphere, the magnetic field it carries winds into an Archimedean spiral. In addition, the radial magnetic field strength is expected to fall off with distance from the Sun, and compressions and rarefactions in the solar wind will develop into clear patterns. Several early studies using data from the Pioneer and Voyager spacecraft had an opportunity to test these claims. Thomas and Smith [1980] used data from Pioneer 10 and 11 to probe the solar wind between 1 and 8.5 AU. They found that the field directions conformed on average to those predicted by the Parker model to an overall accuracy of $1.1^{\circ}$. They also calculated the typical spiral angle at $8.5 \mathrm{AU}$ to be $\sim 83^{\circ}$, and thus suggest that substantial departures are unlikely to be found beyond these distances, as the field becomes almost azimuthal. Similarly the analysis of Voyager and Pioneer 11 data by Burlaga and Ness (1993) shows that the radial variation of the magnetic field strength out to 19 AU is consistent with Parker's model when one considers the latitudinal and temporal variations of the source magnetic field strength and the solar wind speed. 
The Cassini spacecraft approached Saturn's magnetosphere during early 2004, measuring the interplanetary magnetic field (IMF) continuously and sampling solar wind plasma properties when the pointing of the CAPS instrument was favorable. Jackman et al. (2004) then presented a comprehensive study of the structure of the solar wind during the Cassini approach, showing that the IMF was dominated by a clear pattern of corotating interaction region (CIR) compressions and rarefactions, caused in part by the tilt of the Sun's dipole during the declining phase of the solar cycle. This study was followed up by a survey of the IMF parameters (Jackman et al., 2008a) which indicated that the average spiral angle upstream of Saturn is $\sim 83^{\circ}$, agreeing very closely with the predictions of the Parker model. Data from Cassini (declining phase to solar minimum) were then combined with older data from Pioneer and Voyager to build a picture of the solar wind character across different stages of the solar cycle (Jackman and Arridge, 2011). 


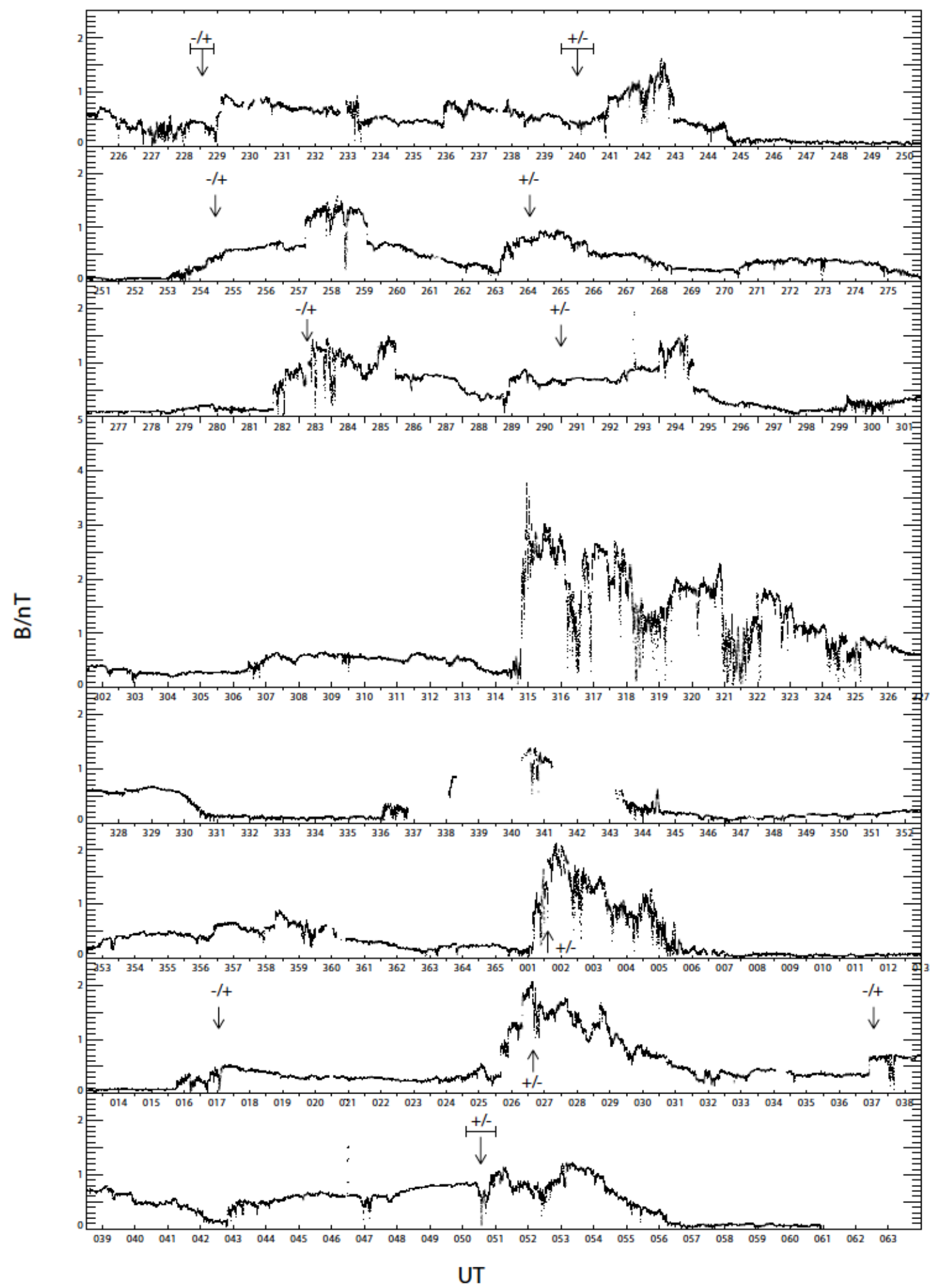

Figure 15: IMF strength measured by Cassini from 2003 day 225 to 2004 day 63. Each panel represents one solar rotation (25.5 days). Crossings of the heliospheric current sheet are denoted by arrows. The pattern of compressions and rarefactions is evident. (from Jackman et al., 2004). 


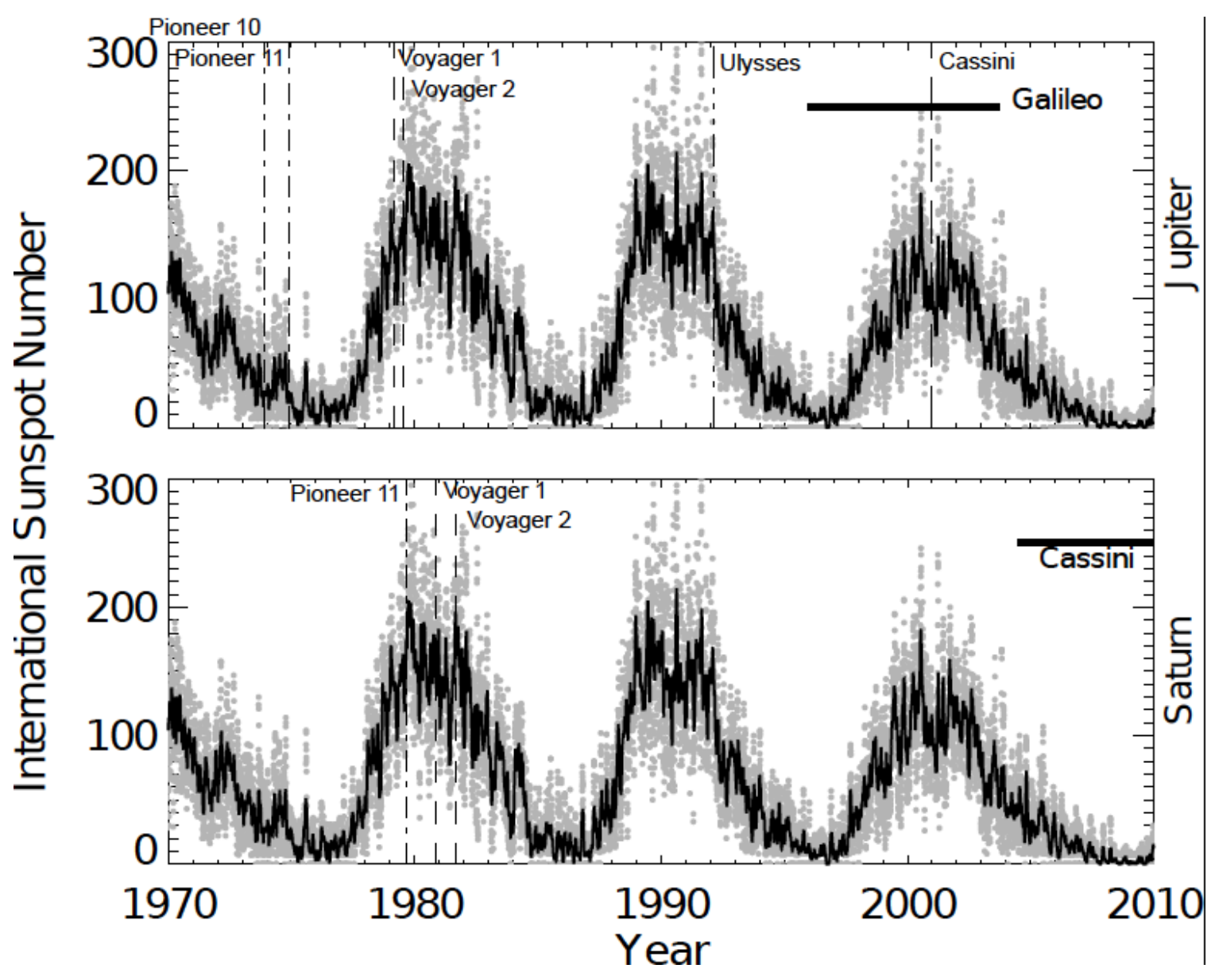

Figure 16: Sunspot number from 1970-2010. The timings of the closest approach of spacecraft to Jupiter (top panel) and Saturn (lower panel) are marked. (from Jackman and Arridge, 2011).

Once the solar wind reaches Saturn's magnetopause it can shape the boundary and the IMF can merge with the planetary field via magnetic reconnection, allowing the transfer of mass, energy and momentum to the system. The extent to which dayside reconnection operates is a topic of intense debate (e.g. Masters et al., 2014 and references therein). The reconnection rate is modulated in some manner by the orientation and magnitude of the IMF. Conflicting early studies have indicated that reconnection is (i) feasible and can be important (e.g. Huddleston et al., 1997, Grocott et al., 2009), or (ii) that reconnection can be suppressed by the high Mach number regime at Saturn (e.g. Scurry and Russell, 1991). Some evidence for dayside reconnection has been presented (McAndrews et al., 2008), while other studies claim its role is rather limited [Lai et al., 2012]. More recently, work has focused on the factors that may govern the reconnection rate, such as the plasma beta (Masters et al., 2012; Desroche et al., 2013), as well as cusp observations at Saturn (Jasinski et al., 2014). This is certainly an ongoing area of research. If/when dayside reconnection is active at Saturn's magnetopause, the theoretical work of Badman and Cowley (2007) has indicated that it can have a very important impact on the dynamics of the Saturnian system, particularly in the outer regions of the magnetosphere. 


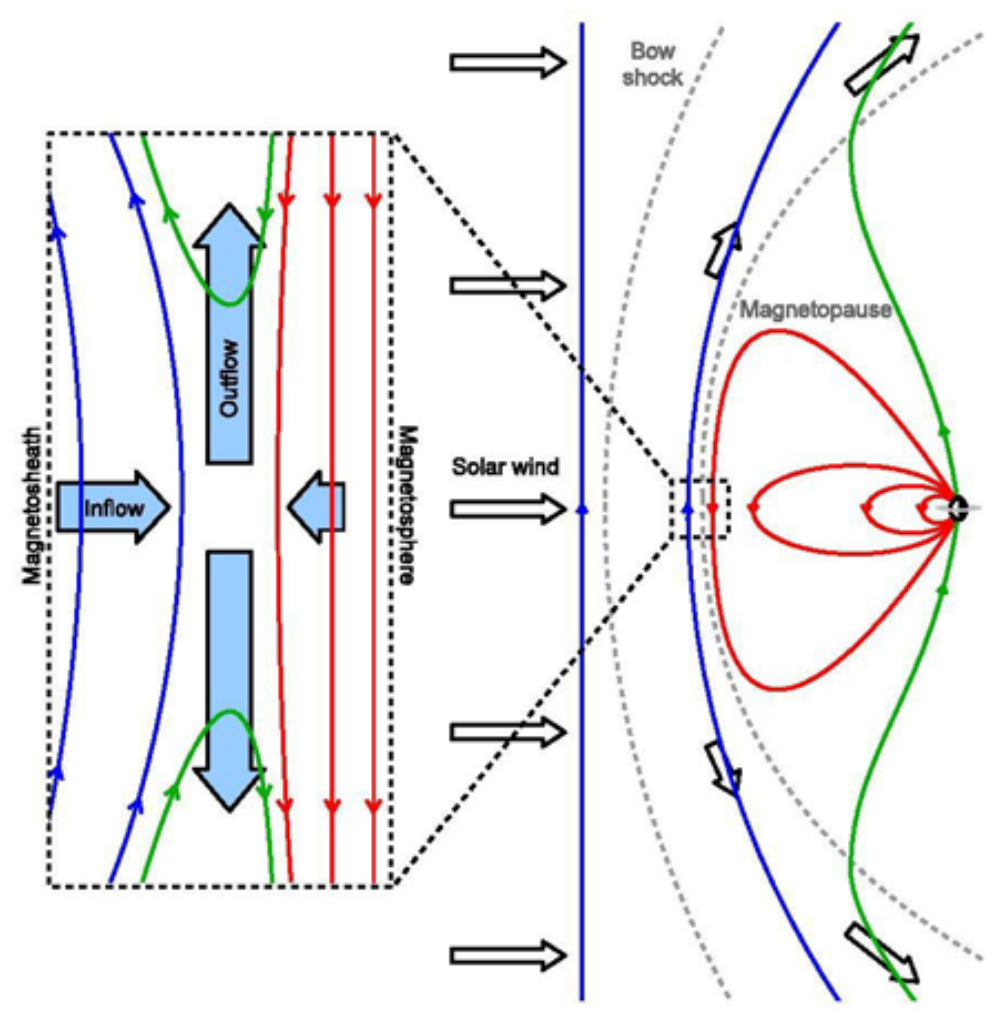

Figure 17: An illustration of magnetic reconnection at Saturn's magnetopause for (rare) perfectly northward interplanetary magnetic field. Interplanetary (solar), planetary, and reconnected (open) magnetic field lines are shown in blue, red, and green, respectively. (from Masters et al., 2014)

\section{Sources for high energy particles}

\subsection{Introduction}

The composition of suprathermal and energetic magnetospheric ions contains partial information on relative plasma source strengths, as does that for the thermal plasma. Although the terms thermal, suprathermal, and energetic are not associated with well-defined energy ranges, in this discussion we will apply them to $<10 \mathrm{keV}, 10-200 \mathrm{keV}$, and $>200 \mathrm{keV}$ particle populations, respectively. The processes of acceleration from the lower energies potentially can alter the composition. Energization also frequently involves transport, so that the spatial distribution of the various energetic species is much less likely to pinpoint source locations than is that of the thermal plasma. Composition measurements at higher energies also have some advantages. In some cases, the mass per charge or mass resolution is better, and identification ambiguities that occur when two species have the same mass per charge can be resolved with techniques available at higher energies.

As is the case at other planets, the most energetic component of the high-energy charged particle population at Saturn is partly produced by the Cosmic Ray Albedo Decay (CRAND) mechanism, 
which is therefore not connected to the composition of these charged particles at lower energies. We will devote below a separate paragraph to the description of this CRAND source.

Before Cassini entered into Saturn orbit in July 2004, composition measurements had only been made for the thermal plasma and at quite high energies $(\sim \mathrm{MeV})$ during the Pioneer 11 and Voyager 1 and 2 flybys. Pioneer 11 reported the presence of protons and helium nuclei at energies $>0.5 \mathrm{MeV} /$ nucleon (e.g., Simpson et al, 1980). Frank et al. (1980) analyzed Pioneer 11 plasma data and concluded from indirect evidence that, in addition to protons present throughout the magnetosphere, a torus of heavy ions existed inside the orbit of Rhea. They identified these ions as most likely being oxygen in the +2 and +3 charge states (the charge state identification turned out not to be correct). Voyager 1 and 2 reported the presence of both protons and an abundant heavy ion (either $\mathrm{N}^{+}$or $\mathrm{O}^{+}$) at thermal energies (Lazarus and McNutt, 1983). Further analysis of the Voyager plasma data favored the $\mathrm{O}^{+}$interpretation (Richardson, 1986). At higher energies above $0.5 \mathrm{MeV}$ /nucleon, Hamilton et al (1983) identified $\mathrm{H}^{+}, \mathrm{H}_{2}{ }^{+}, \mathrm{H}_{3}{ }^{+}$, and $\mathrm{He}$ along with a small amount of $\mathrm{C}, \mathrm{N}$, and $\mathrm{O}$ (without charge state identification for any of the species). Later, the remote detection of an OH torus (Shemansky et al., 1993) led to detailed modeling by Jurac and Richardson (2005) with the conclusion that Saturn's magnetosphere must have a strong source of water with a maximum around the orbit of Enceladus.

The Cassini measurements of suprathermal ion composition have been possible because of development of time-of-flight methods in space instrumentation (Gloeckler et al., 1990) since the Pioneer and Voyager missions. The results reviewed in this section are largely measurements made in the suprathermal energy range by the Cassini Charge Energy Mass Spectrometer (CHEMS) instrument (instrument described by Krimigis et al., 2004). Suprathermal composition measurements were not made by either Pioneer 11 or the two Voyagers.

Data from Saturn Orbit Insertion, shown in the right panel of Fig. 18, immediately showed differences with observations made by the same instrument during Cassini's Earth and Jupiter flybys (left two panels). Although $\mathrm{H}^{+}, \mathrm{He}^{+}, \mathrm{He}^{++}$and $\mathrm{O}^{+}$are present in all three magnetospheres, Saturn shows a broader $\mathrm{O}^{+}$distribution, indicative of the presence of additional water products $\left(\mathrm{OH}^{+}, \mathrm{H}_{2} \mathrm{O}^{+}\right.$, and $\left.\mathrm{H}_{3} \mathrm{O}^{+}\right)$along with the molecular ions $\mathrm{H}_{2}^{+}$and $\mathrm{O}_{2}^{+}$. The open question left from the Voyager flybys concerning the identity of the dominant heavy ion was immediately answered; it was $\mathrm{O}^{+}$and the other water products. Because of Titan's nitrogen atmosphere, the relative absence of $\mathrm{N}^{+}$, found to be much less abundant in Saturn's magnetosphere than even in Earth's, was surprising to many. The lack of sulfur ions, present at Jupiter as a result of Io's volcanism, was not surprising. 


\section{Cassini/MIMI/CHEMS}

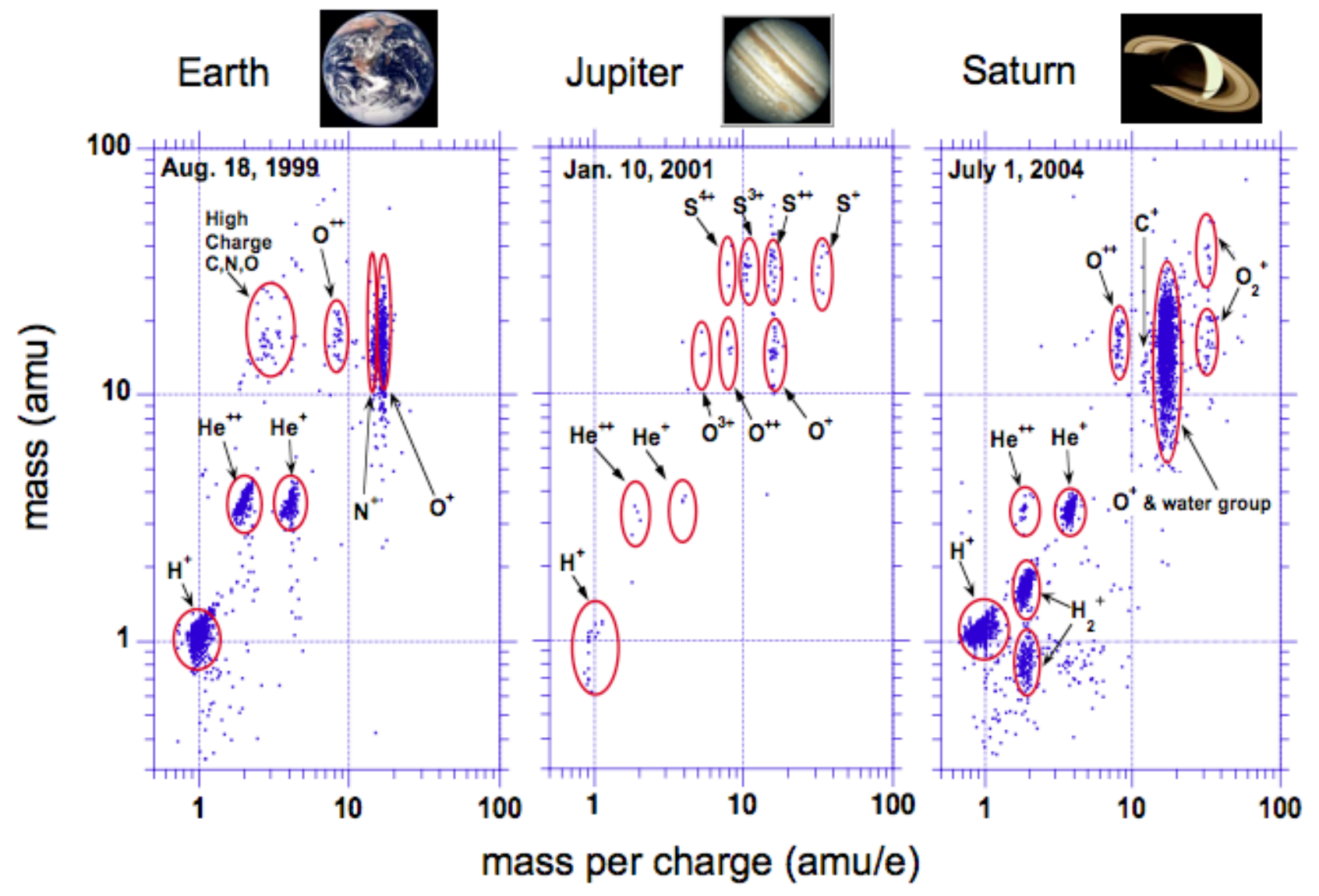

Figure 18: Suprathermal ion composition data from the Cassini/CHEMS instrument from three planetary magnetospheres. The data were obtained during the Cassini flybys of Earth and Jupiter and during SOI at Saturn. The composition differs among the planets and is indicative of different plasma sources. The time, radial distances, and energy intervals for the three data sets are the following: Earth 0225-0250, Day 230, 1999, $\mathrm{R}=6.2-10.1 \mathrm{R}_{\mathrm{E}}$, dayside, 55-167 keV/e; Jupiter 1916-2100, Day 10, 2001, R=204 R, dusk flank, 94-97 keV/e; Saturn 0800 Day 182 1610 Day $183,2004, \mathrm{R}=15.0 \mathrm{R}_{\mathrm{S}}$ inbound to $10.8 \mathrm{R}_{\mathrm{S}}$ outbound, $83-220 \mathrm{keV} / \mathrm{e}$. (from Hamilton et al., 2005)

\subsection{Overview}

Figure 19 presents a mass per charge (M/Q) histogram of Saturn data accumulated by the CHEMS instrument over a $\sim 2$-year period showing multiple species. Since the detection efficiency and minimum energy both vary with mass (see figure caption), the apparent relative abundances in Fig. 19 are only qualitative. CHEMS uses electrostatic deflection to determine an ion's energy per charge (E/Q), along with a time-of-flight (TOF) measurement and a kinetic energy measurement (E) in solid state detectors (SSDs) to determine mass per charge M/Q and mass M. Although CHEMS' entire energy range is 3-220 keV/e, the SSDs do not trigger for incident ions in the lower portion of that range (see caption). When there is no energy signal, 
only M/Q is determined. All the events in Figure 19 had an energy signal, so both $\mathrm{M} / \mathrm{Q}$ and $\mathrm{M}$ are determined, and species such as $\mathrm{H}_{2}^{+}$and $\mathrm{He}^{++}$, which have the same value of M/Q and coincide in Figure 19, can be separated.

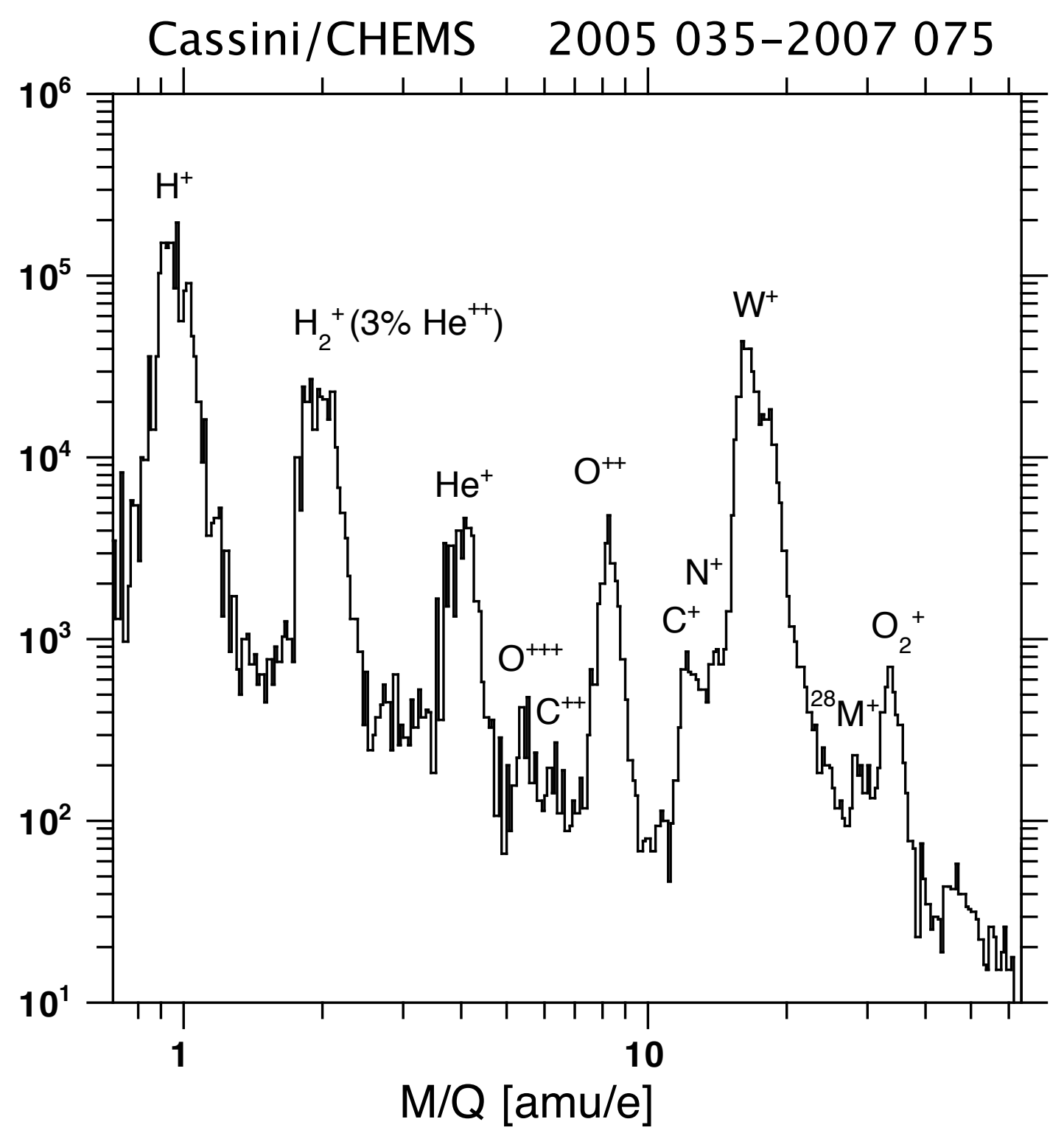

Figure 19 : Two-year sum of suprathermal ion composition in Saturn's magnetosphere from the Cassini/CHEMS instrument. Only events that include both a time-of-flight measurement and an energy signal from the solid state detectors (SSDs) are included (lowest background data). The minimum energy to trigger the SSDs increases with mass. Approximate energy ranges are 25$220 \mathrm{keV}$ for $\mathrm{H}^{+}$and $55-220 \mathrm{keV}$ for $\mathrm{W}^{+}$. Apparent relative abundances are only qualitative. (from Mauk et al., 2009) 
Even though quantitative comparisons cannot be made from Figure 19, the three most abundant species in Saturn's magnetosphere, $\mathrm{H}^{+}, \mathrm{W}^{+}$(the water group ions comprising $\mathrm{O}^{+}, \mathrm{OH}^{+}, \mathrm{H}_{2} \mathrm{O}^{+}$, and $\mathrm{H}_{3} \mathrm{O}^{+}$), and $\mathrm{H}_{2}{ }^{+}$, stand out. The dominant sources of these three species, according to current thought, are all local, but different. The water products originate predominately from the Enceladus plumes with a source rate of $1-4 \times 10^{28} \mathrm{~s}^{-1}$ (Burger et al., 2007; Jia et al., 2010; Dong et al., 2011). The $\mathrm{H}^{+}$has several potential sources including the solar wind and dissociation of the Enceladus water, but the largest source appears to be ionization of the extensive neutral $\mathrm{H}$ cloud arising from Saturn's atmosphere (e.g., Melin et al., 2009). The strongest source of $\mathrm{H}_{2}$ appears to be Titan's atmosphere $\left(\sim 1 \times 10^{28} \mathrm{~s}^{-1}\right.$, Cui et al.. 2008), although photo-dissociation of water can be important inside $\sim 6 \mathrm{R}_{\mathrm{S}}$ (Tseng et al., 2011).

The relative abundances of the major and minor suprathermal species in the ring current $(\mathrm{L}=7$ 16) are given in Table 1. The table lists ratios of each species to $\mathrm{H}^{+}$along with their fractional abundances in the total suprathermal population, listed from highest to lowest. The energy range is $27-220 \mathrm{keV} / \mathrm{e}$ for most of the species, including the most abundant, but differ for some of the rarer species. The makeup of the water group $\mathrm{W}^{+}$is given in the first column. We discuss several of the species in more detail later in this section. This discussion does not include the multi-MeV ions comprising Saturn's permanent radiation belts at $<4 \mathrm{R}_{\mathrm{S}}$ (Paranicas et al., 2008).

\subsection{Spatial Variations}

Figure 20 presents the radial profiles of the partial number densities (PNDs) of six suprathermal species (73-110 keV/e) (left panel) and their fractional abundances (FAs) (right panel) (DiFabio 2012). These ions comprise the more energetic portion of Saturn's ring current (Sergis et al., 2007) and have peak densities at L 10 in this energy range. The similar profiles of the different species are an indication of common acceleration processes, probably involving a combination of outer magnetospheric and tail processes [flux tube interchange, reconnection, etc.] (e.g. Mauk et al., 2005; Mitchell et al., 2005; Rymer et al., 2009) and inward radial diffusion with rapid losses in the Enceladus neutral cloud causing the decreases inside L 9 (Paranicas et al., 2008). 

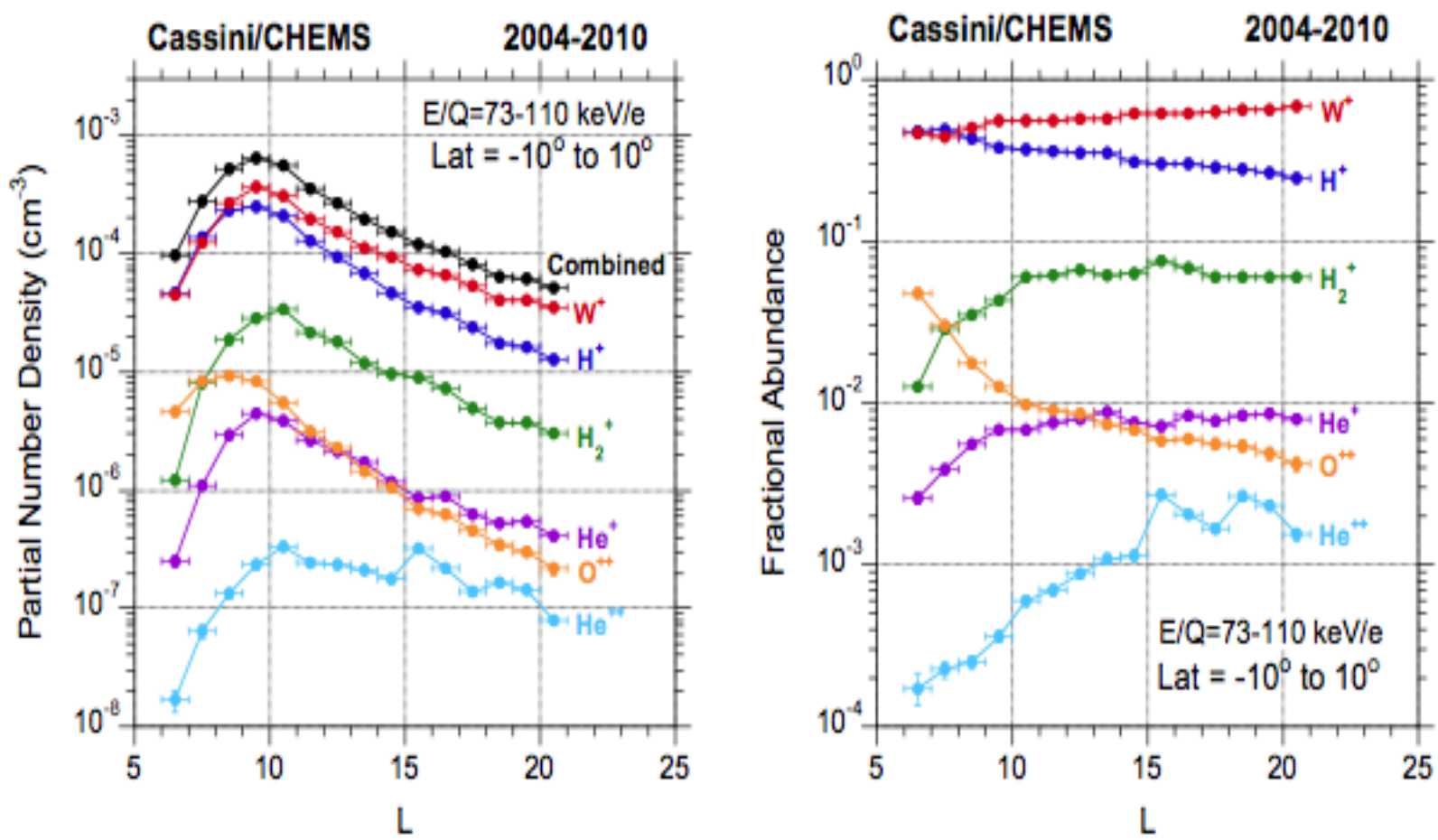

Figure 20 : Average (2004-2010) partial number densities (left) and fractional abundances (right) for ions in the 73-110 keV/e range are plotted versus dipole L. (from DiFabio, 2012)

Differences among the species inside $\mathrm{L}=10$ are largely attributable to differences in charge exchange lifetimes and other loss processes (DiFabio, 2012). The relative increase of the $\mathrm{O}^{++}$ FA at lower L-shells arises from its increased production from $\mathrm{O}^{+}$in the region of higher density neutrals and plasma electrons. The rapid decline in the $\mathrm{He}^{++}$FA inside $\mathrm{L}=15$ is largely due to single electron capture from neutrals, producing more $\mathrm{He}^{+}$.

\subsection{Temporal Variations}

The long-term temporal variations in suprathermal ion intensities from 2004 to the end of 2010 have been studied by DiFabio et al. (2011). Fig. 21 presents PNDs (left panel) and FAs (right panel) of the various species. These measurements were made in the near-equatorial ring current (dipole $\mathrm{L}=7-16$ ). Overall variations are, in general, quite modest ( factor 2 ) for the major species. In particular, the relative constancy of the $\mathrm{W}^{+}$PND led DiFabio et al. (2011) to conclude that the Enceladus plume source cannot have variations much larger than that during this period when averaged over six months to a year. Shorter time variations are certainly possible (e.g., Smith et al., 2010; Jia et al., 2010) and would not show up in these long averages. The variations in $\mathrm{He}^{++}$and $\mathrm{He}^{+}$are somewhat larger. They show decreases in 2009-2010 near solar minimum that are not seen in the other species. 

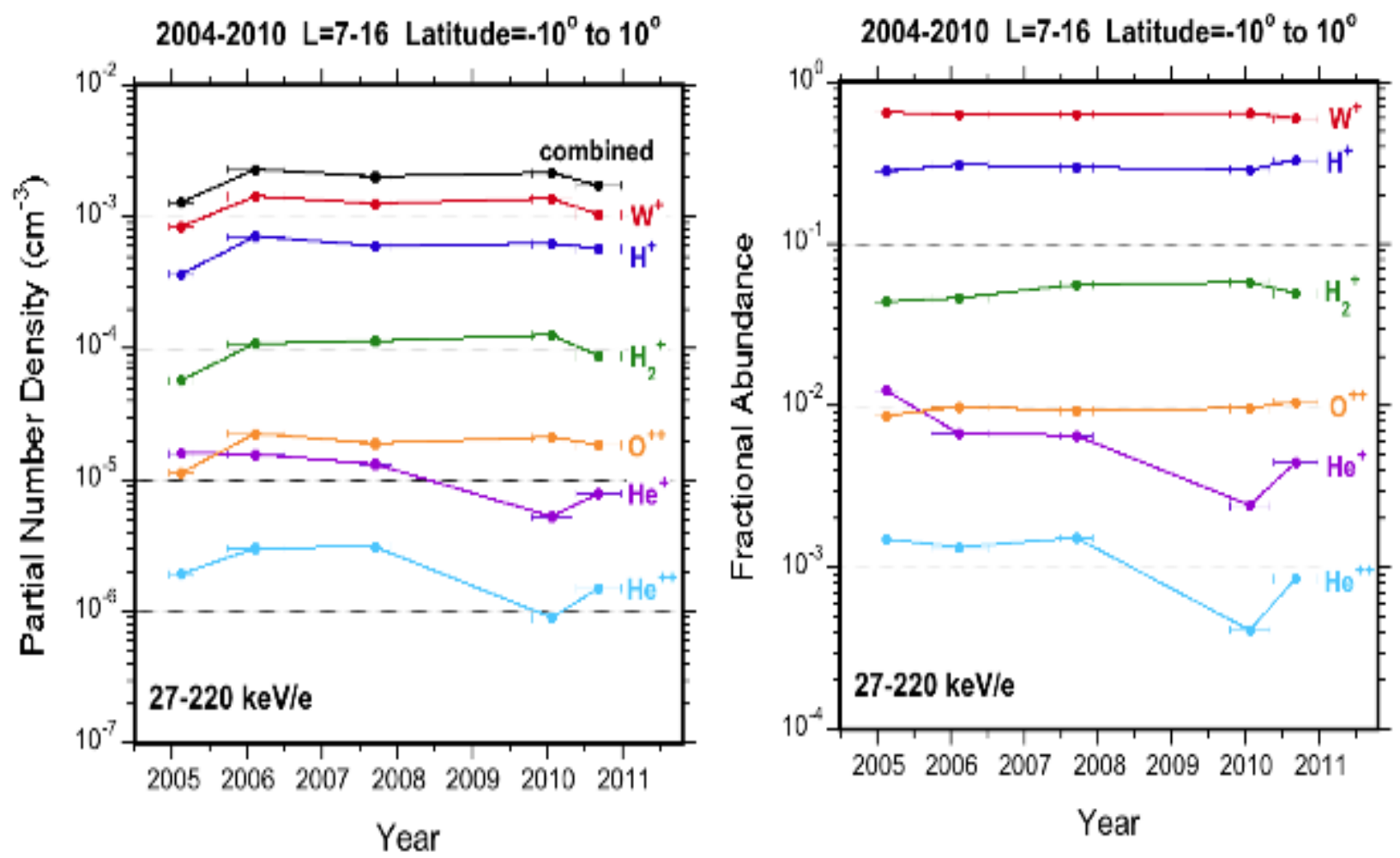

Figure 21 : Suprathermal ion (27-220 keV/e) partial number densities and fractional abundances from Cassini/CHEMS for five long averaging periods from late 2004 to the end of 2010. Statistical error bars are smaller than the data point symbols. (from DiFabio et al., 2011)

\section{- Water Group lons ( $\left.\mathbf{W}^{+}\right)$}

The water group ions are the most abundant species throughout most of Saturn's magnetosphere at both thermal and suprathermal energies (Thomson et al., 2010; DiFabio 2012). In the CHEMS data, the four species comprising the suprathermal water group have fairly broad distributions in measured $\mathrm{M} / \mathrm{Q}$. That fact combined with some instrumental issues has allowed an accurate determination of their relative abundances in only a limited energy range around 96 $\mathrm{keV}$. Figure 22 taken from DiFabio (2012), indicates fits to the four $\mathrm{W}^{+}$components. As listed in Table $1 \mathrm{O}^{+}(53 \%)$ dominates the suprathermal $\mathrm{W}^{+}$with $\mathrm{OH}^{+}$and $\mathrm{H}_{2} \mathrm{O}^{+}$each present at somewhat less than half that amount $(22 \%) . \mathrm{H}_{3} \mathrm{O}^{+}$is present in trace quantities $(2.8 \%)$, although its abundance is least well determined. 


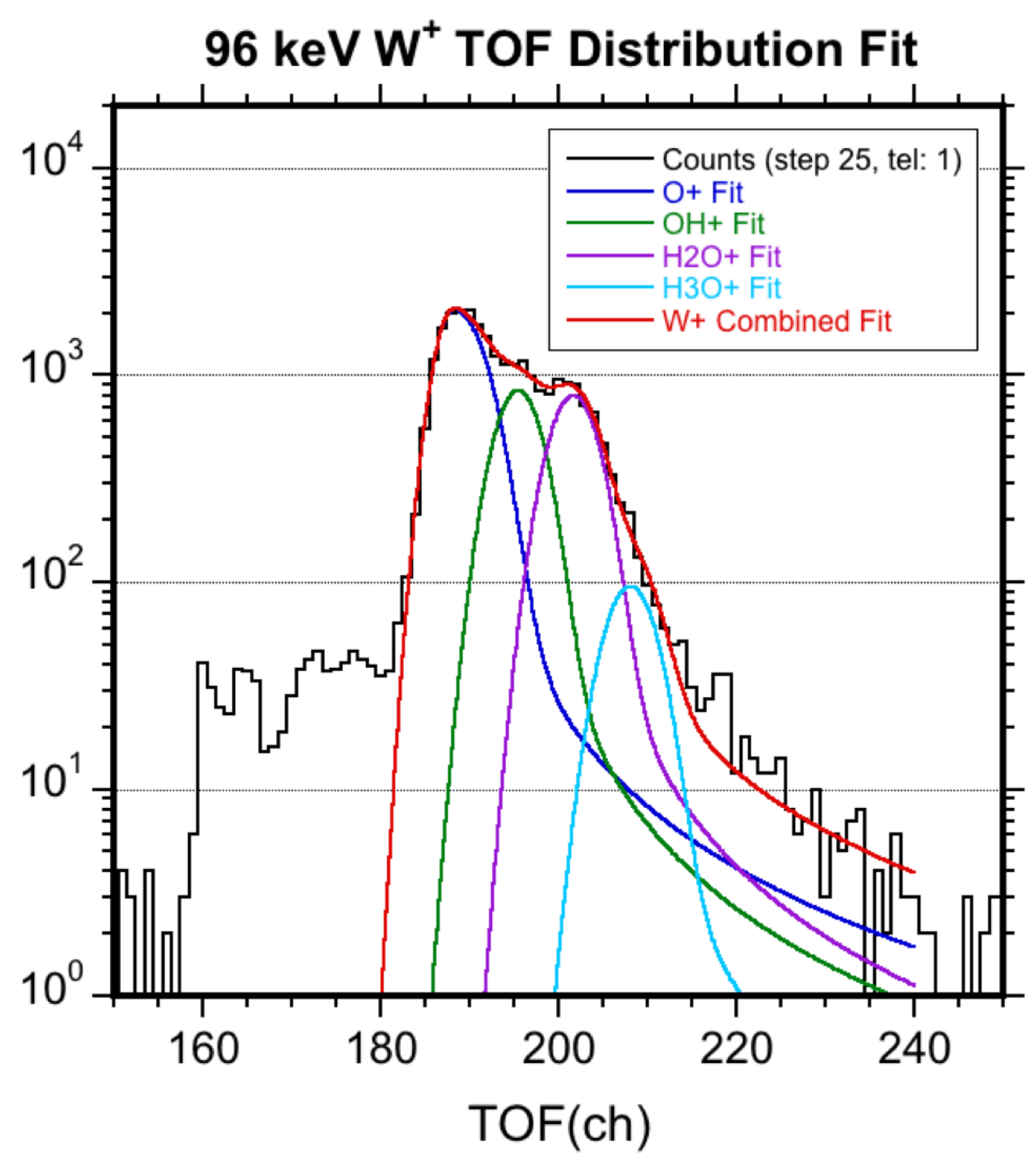

Figure 22 : Best fits to the water group ion distribution in the $94-97 \mathrm{keV}$ energy range. The fits to each of the four individual species are shown along with the sum of the four fits in red. The data are from a long average from near equatorial (within $10^{\circ}$ ) passes through the ring current ( $\mathrm{L}=7-16$ ) from late 2004 to the end of 2010. Cassini/CHEMS telescope 1 was used. (from DiFabio, 2012)

$\mathrm{H}^{+}, \mathrm{He}^{+}$, and $\mathrm{He}^{++}$

$\mathrm{He}^{++}$has no known local source and comes from the solar wind, and $\mathrm{He}^{+}$is thought to originate from interplanetary pickup ions. Low magnetospheric $\mathrm{He}^{++} / \mathrm{H}^{+}$ratios, compared to $4 \%-5 \%$ in the solar wind, were noted at $\mathrm{MeV}$ energies during the Pioneer 11 and Voyager flybys [Simpson et al., 1980; Hamilton et al., 1983], with the conclusion that the $\mathrm{H}^{+}$is mostly of local origin. Cassini's measurements at suprathermal energies lead to a similar conclusion. Table 1 lists that ratio as 0.0029 in the main ring current $(L=7-16)$, more than a factor of 10 less than the solar 
wind value. Fig. 20 shows that the ratio has a higher value (0.0074) in the outer magnetosphere $(\mathrm{L}=15-21)$, which is still a factor of 6 less than the solar wind value. That value is probably better for comparison since it avoids the inner region where $\mathrm{He}^{++}$is preferentially lost. This would imply that about $84 \%$ of the magnetospheric $\mathrm{H}^{+}$is of local origin and $\sim 16 \%$ originates in the solar wind.

A crude estimate of the solar wind source rate can be made by assuming a small fraction of incident solar wind ions enter the magnetosphere (a range of 0.001 to 0.01 has been used at Earth). With a cross-sectional radius of $25 \mathrm{R}_{\mathrm{S}}, 400 \mathrm{~km} / \mathrm{s}$ solar wind speed, and an average solar wind density of $0.05 \mathrm{~cm}^{-3}$, one obtains a solar wind $\mathrm{H}^{+}$source rate of $\sim 1.6 \times 10^{26} \mathrm{~s}^{-1}$ to $1.6 \times 10^{27}$ $\mathrm{s}^{-1}$. The calculated solar wind source rate for $\mathrm{He}^{++}$is then $\sim 7 \times 10^{24} \mathrm{~s}^{-1}$ to $7 \times 10^{25} \mathrm{~s}^{-1}$. This implies a local source of $\mathrm{H}^{+}$in the range of $8 \times 10^{26} \mathrm{~s}^{-1}$ to $8 \times 10^{27} \mathrm{~s}^{-1}$.

\section{$-\mathrm{C}^{+}$and $\mathrm{N}^{+}$}

Singly charged carbon and nitrogen are interesting trace species $\left(<1 \%\right.$ of $\left.\mathrm{W}^{+}\right)$in the suprathermal particle population. Before Cassini's arrival at the planet, it had been expected that $\mathrm{N}^{+}$might be a major species in Saturn's magnetosphere because it constitutes 95\% of Titan's atmosphere. However, DiFabio (2012) found average ratios of $\mathrm{C}^{+}$and $\mathrm{N}^{+}$to $\mathrm{W}^{+}$of only 0.0055 and 0.0078 , respectively, in the 127-220 keV range (see also preliminary CHEMS results in Table 11.3 of Mauk et al., 2009). Fig. 23 shows the radial variations of the $\mathrm{W}^{+}, \mathrm{N}^{+}$, and $\mathrm{C}^{+}$PNDs from a slightly more restrictive energy range. The three species have similar radial profiles that offer little information about source locations. Although Titan would potentially be a source of both $\mathrm{N}^{+}$and $\mathrm{C}^{+}\left(\mathrm{CH}_{4}\right.$ is the second most abundant species in Titan's atmosphere), measurements of the thermal plasma have indicated that Enceladus is the more likely source of $\mathrm{N}^{+}$(Smith et al., 2007). $\mathrm{C}^{+}$has not yet been observed in the thermal plasma. 


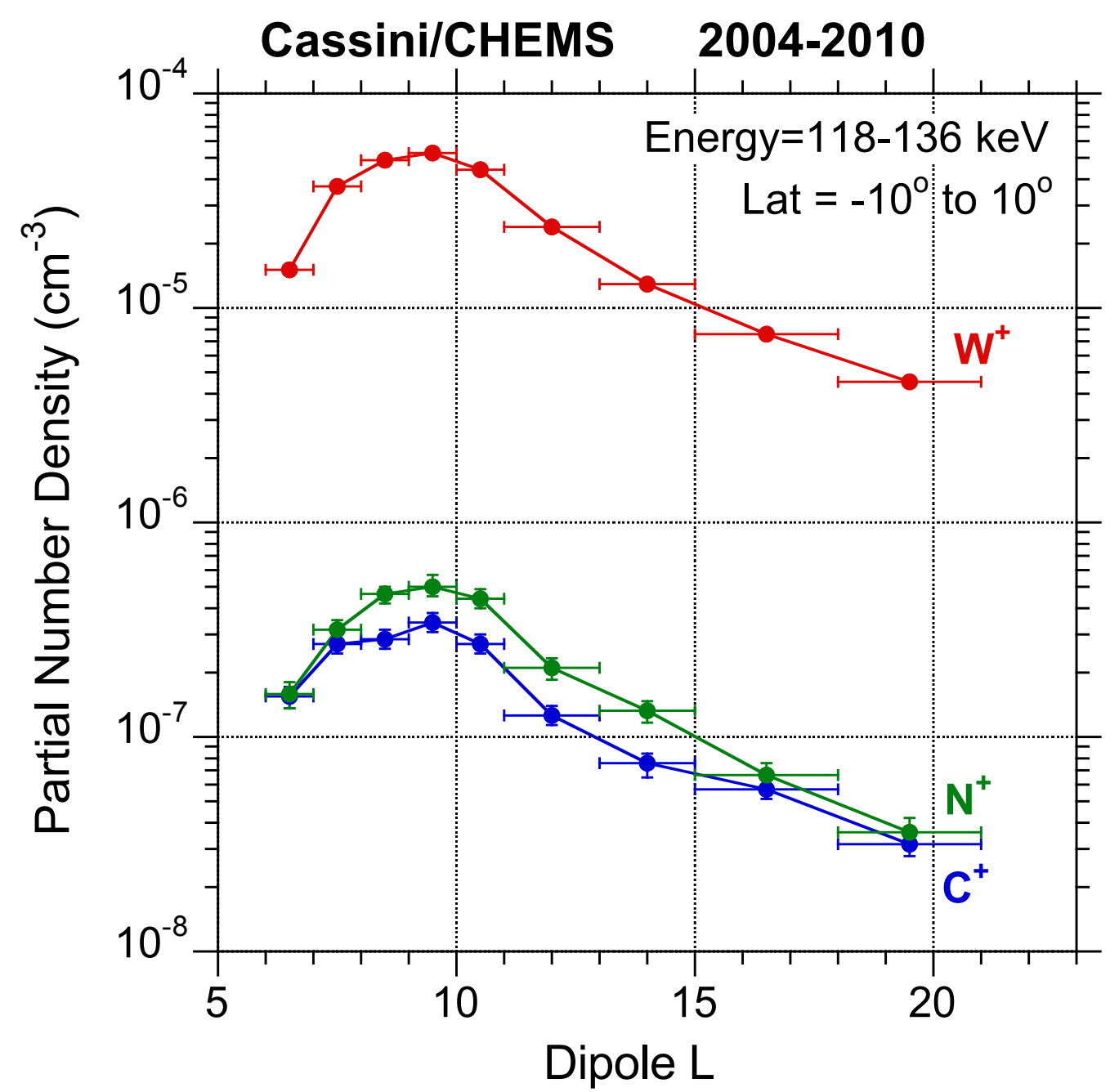

Figure 23 : Radial profiles of the $118-136 \mathrm{keV} \mathrm{C}^{+}, \mathrm{N}^{+}, \mathrm{W}^{+}$partial number densities. The data are averaged over the period late 2004 to the end of 2010. (from DiFabio, 2012)

\section{$-\mathrm{O}_{2}{ }^{+}$and ${ }^{28} \mathrm{M}^{+}$}

Suprathermal molecular ions $\mathrm{O}_{2}{ }^{+}$and ${ }^{28} \mathrm{M}^{+}$(leading candidates for ${ }^{28} \mathrm{M}^{+}$are $\mathrm{N}_{2}{ }^{+}$and $\mathrm{CO}^{+}$) have been investigated by Christon et al. (2013). Figure 24 (taken from that paper) shows the PNDs of $83-167 \mathrm{keV} \mathrm{W}^{+}, \mathrm{O}_{2}{ }^{+}$, and ${ }^{28} \mathrm{M}^{+}$in the top panel over the late 2004 to early 2012 time period. There are $\sim$ factor 2 variations in all three species. However, the ratios of $\mathrm{O}_{2}{ }^{+}$and ${ }^{28} \mathrm{M}^{+}$to $\mathrm{W}^{+}$ (bottom panel) show smoother, better organized time variations. Christon et al. (2013) have interpreted the $\mathrm{O}_{2}{ }^{+} / \mathrm{W}^{+}$ratio variation as evidence for a varying ring source strength of $\mathrm{O}_{2}{ }^{+}$that depends on the degree of solar illumination (insolation) of Saturn's rings. Tseng et al. (2010) have modeled such a seasonally varying $\mathrm{O}_{2}{ }^{+}$source. The $\mathrm{O}^{+} / \mathrm{W}^{+}$follows the dashed insolation curve from the beginning of mission until Saturn equinox, when sunlight strikes the rings edge on and insolation is minimum. Recovery of the ratio only begins after a year and a half at baseline values and falls below the $100 \%$ (same as pre-equinox) insolation curve. 
Although the ${ }^{28} \mathrm{M}^{+} / \mathrm{W}^{+}$ratio initially shows a seasonal decrease, by 2007 it hits a baseline minimum value that is maintained, with some variations, until 2012. The ${ }^{28} \mathrm{M}^{+}$, and even $\mathrm{O}_{2}{ }^{+}$ with its 1.5 -year extended minimum, are not entirely seasonally varying and probably have multiple sources.

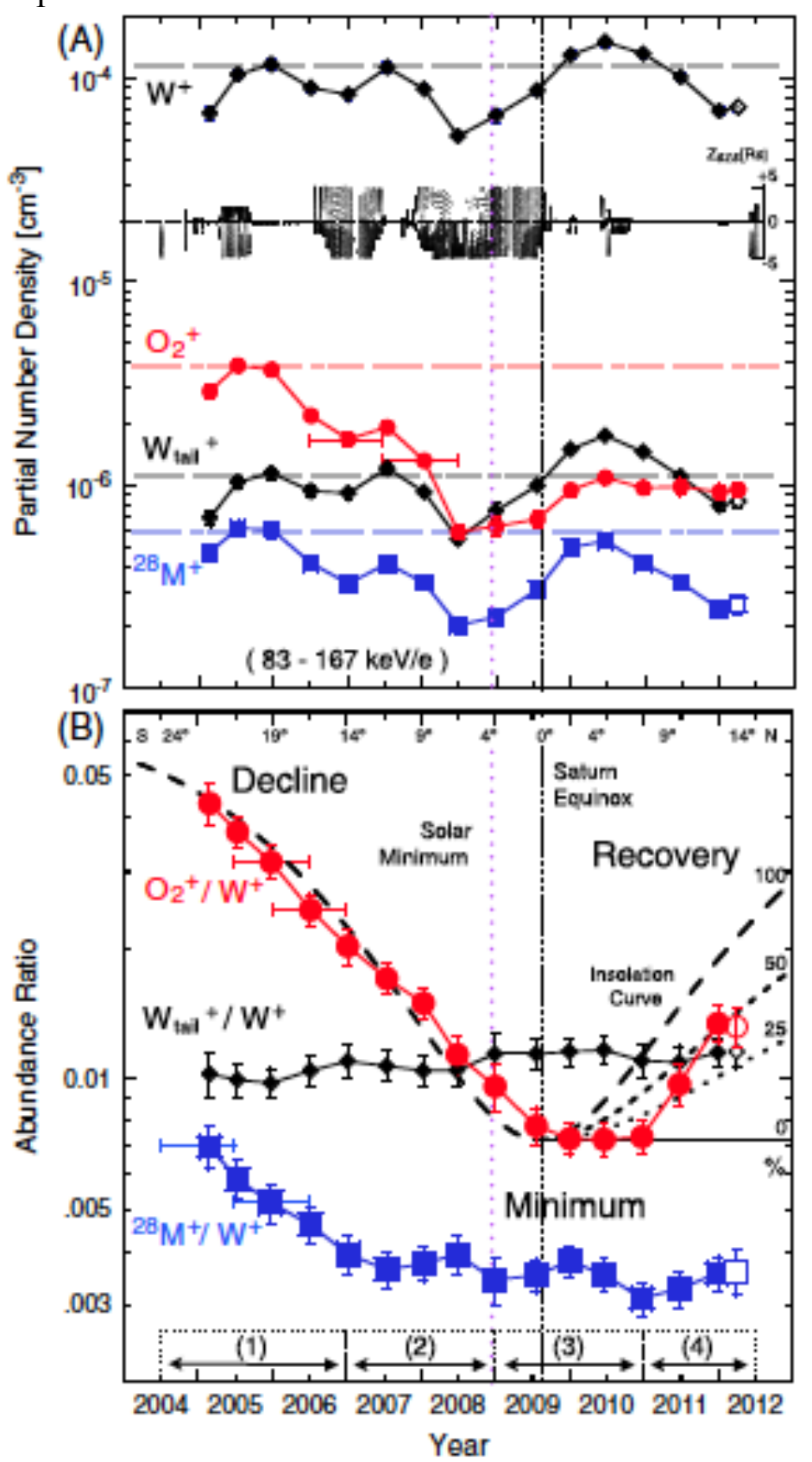

Figure 24 : (A) One-year moving averages of $\mathrm{W}^{+}, \mathrm{O}_{2}{ }^{+}$, and ${ }^{28} \mathrm{M}^{+}$partial number densities (along with $\mathrm{W}_{\text {tail }}{ }^{+}$to track possible time variations in the background contributions of the much more abundant $\mathrm{W}^{+}$ions to the rarer species) in the $83-167 \mathrm{keV} / \mathrm{e}$ energy range. (B) Abundance ratios relative to $\mathrm{W}^{+}$remove spatial/temporal variations common to all species. The dashed lines show the time variation of Saturn ring insolation. The curve is matched to the $\mathrm{O}_{2}{ }^{+} / \mathrm{W}^{+}$decline. During 
recovery curves representing $100 \%, 50 \%$, and $25 \%$ of pre-equinox insolation are shown. (from Christon et al., 2013)

- $\mathrm{H}_{2}^{+}$and $\mathrm{H}_{3}^{+}$

Energetic ( $>0.5 \mathrm{MeV} /$ nuc) $\mathrm{H}_{3}{ }^{+}$, along with more abundant $\mathrm{H}_{2}{ }^{+}$, was discovered in the Jovian magnetosphere by Voyager 2 (Hamilton et al., 1980). Both Voyager 1 and 2 also detected $\mathrm{H}_{2}{ }^{+}$in Saturn's magnetosphere and Voyager 2 detected a few counts of $\mathrm{H}_{3}{ }^{+}$(Hamilton et al., 1983). At these high energies, at the very upper end of Saturn's ring current population, the $\mathrm{H}_{2}{ }^{+}$abundance was similar to, or somewhat less than, that of helium nuclei, whose charge state was not measured by Pioneer 11 or the Voyagers, and was much less abundant than $\mathrm{H}^{+}(<1 \%)$. The situation is different in the suprathermal energy range in which the average $\mathrm{H}_{2}^{+} / \mathrm{H}^{+}$ratio is $\sim 0.17$ (Table 1). The $\mathrm{H}_{2}^{+} / \mathrm{H}^{+}$ratio is also high (tens of percent) at thermal energies in the outer magnetosphere (Thomsen et al., 2010).

The dominant source of $\mathrm{H}_{2}{ }^{+}$is thought to be Titan (Cui et al., 2008) although other sources may play some role (Tseng et al., 2011). The only identified source for $\mathrm{H}_{3}{ }^{+}$is Saturn's ionosphere, where it is produced by the reaction $\mathrm{H}_{2}^{+}+\mathrm{H}_{2} \rightarrow \mathrm{H}_{3}^{+}+\mathrm{H} \cdot \mathrm{H}^{+}$and $\mathrm{H}_{3}^{+}$dominate Saturn's ionosphere (see Nagy et al., 2009) and can be extracted from the auroral regions and accelerated to produce field-aligned beams and conics. Mitchell et al. (2009) reported the presence of $\mathrm{H}_{3}{ }^{+}$in such a beam at $\sim 10 \%$ the level of $\mathrm{H}^{+}$. Energy spectra of $\mathrm{H}^{+}$and $\mathrm{H}_{3}{ }^{+}$from that event are shown in Fig. 25. 


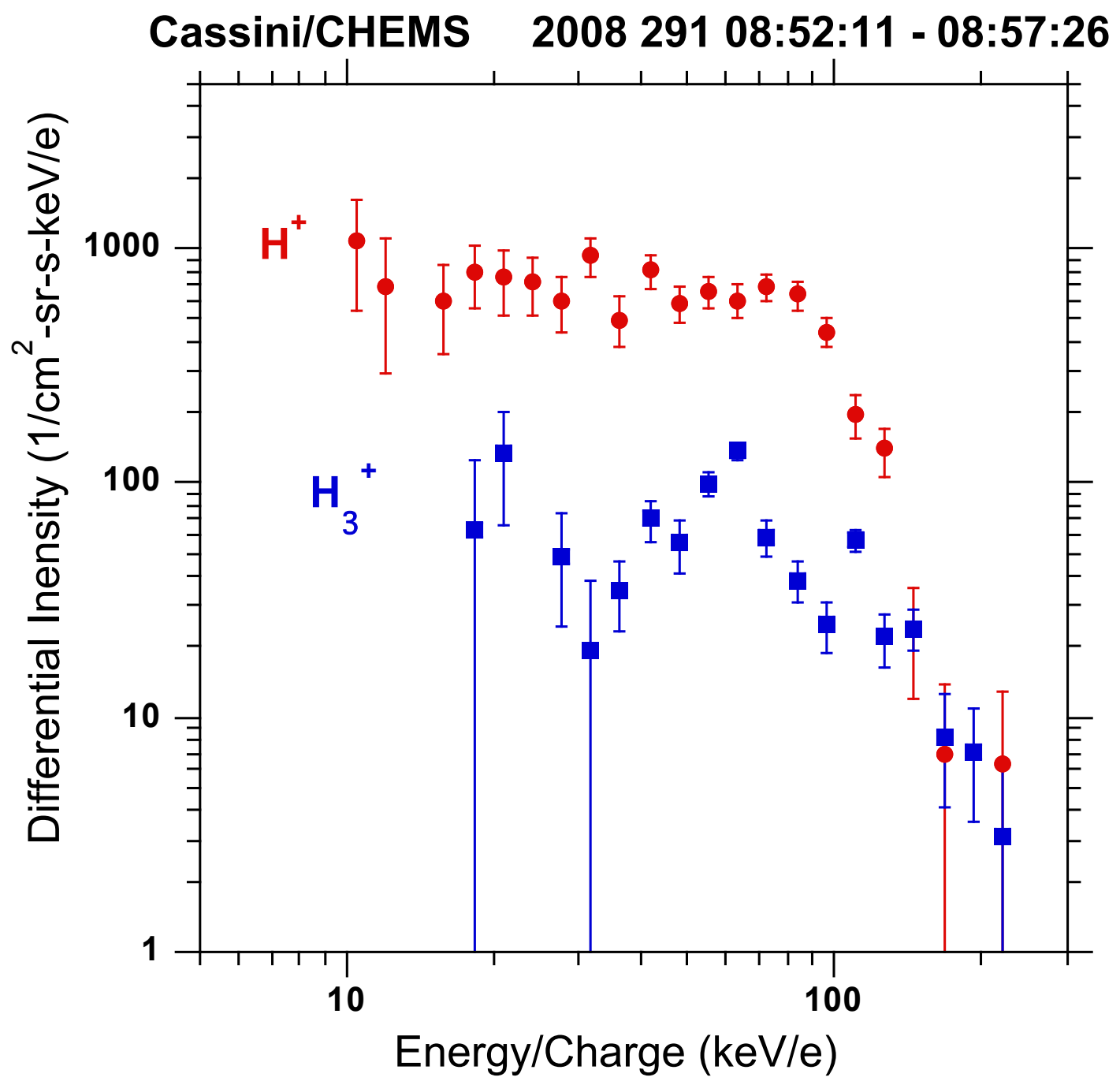

Figure 25 : Energy spectra of $\mathrm{H}^{+}$and $\mathrm{H}_{3}{ }^{+}$observed during $\sim 5$ minutes of a Saturn auroral event during which CHEMS was favorably oriented to observe the nearly field-aligned beam. (from Hamilton et al., 2013)

The abundance of $\mathrm{H}_{3}{ }^{+}$in the more typical ring current population is much lower. Fig. 26 shows PNDs of $\mathrm{H}^{+}$and $\mathrm{H}_{3}{ }^{+}$(36-167 keV) from 37 Cassini passes through the ring current. The average $\mathrm{H}_{3}{ }^{+} / \mathrm{H}^{+}$ratio over this 2005-2012 period was 0.0072 . Whether the sporadic auroral beams are sufficient to feed the ring current is not known. Another possible source is Saturn's polar wind. Glocer et al. (2007) have estimated its contribution to the magnetosphere to be in the range $2.1 \mathrm{x}$ $10^{26}$ to $7.5 \times 10^{27}$ ions/s. In their model the polar wind is comprised of $\mathrm{H}^{+}$and $\mathrm{H}_{3}{ }^{+}$and, depending on the neutral temperature, either species can dominate. The fact that the $\mathrm{H}_{3}{ }^{+} / \mathrm{H}^{+}$ ratio is about the same as the outer magnetospheric $\mathrm{He}^{++} / \mathrm{H}^{+}$ratio discussed above would suggest an $\mathrm{H}_{3}{ }^{+}$source rate of $\sim 7 \times 10^{24} \mathrm{~s}^{-1}$ to $7 \times 10^{25} \mathrm{~s}^{-1}$, assuming the loss rates of $\mathrm{H}_{3}{ }^{+}$and $\mathrm{He}^{++}$are not drastically different. This source rate range is a factor of 3 to 30 below the Glocer et al. (2007) lower limit. 


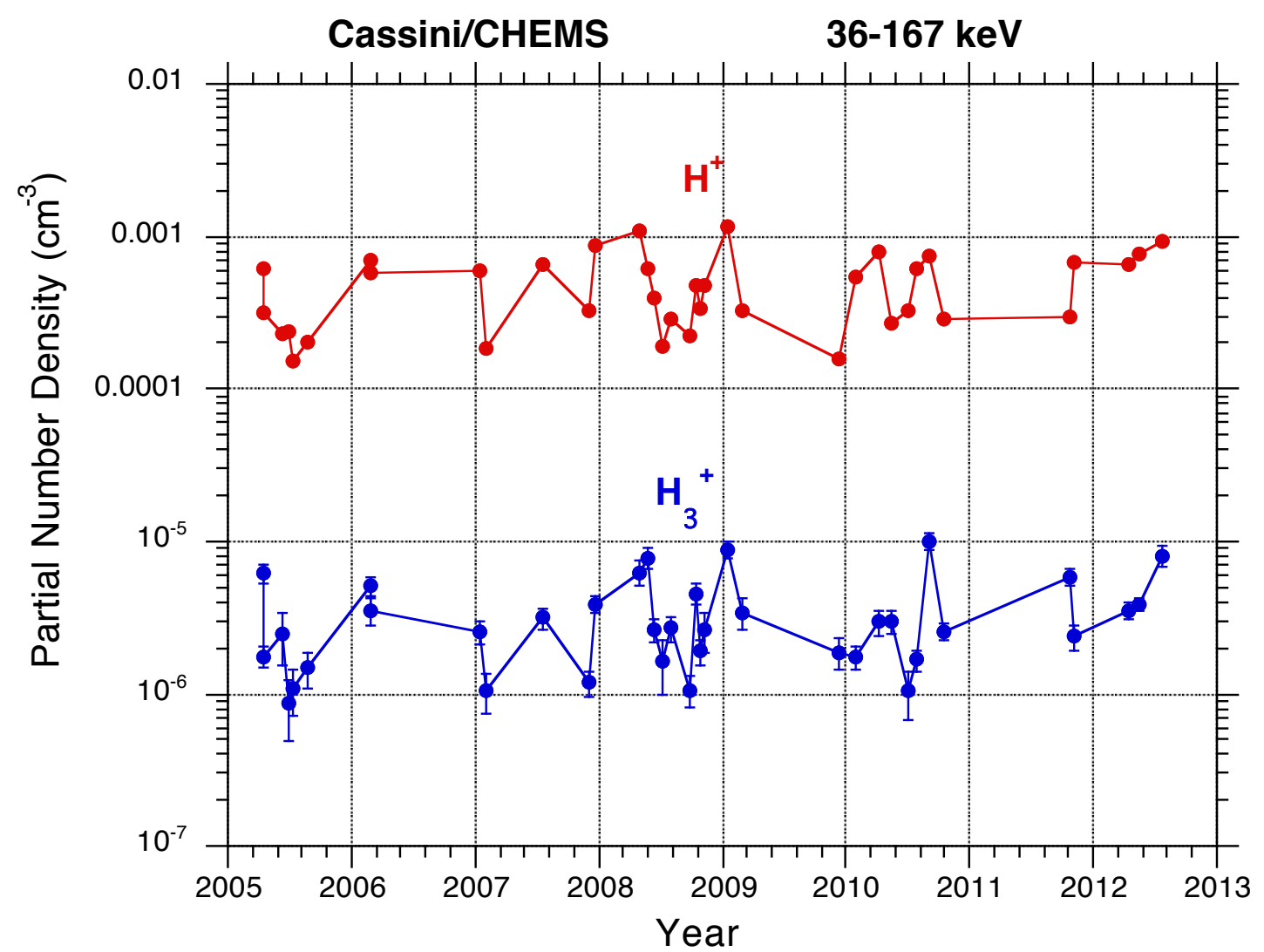

Figure 26 : Suprathermal (36-167 keV) partial number densities of $\mathrm{H}^{+}$and $\mathrm{H}_{3}{ }^{+}$observed by Cassini/CHEMS during 37 passes through Saturn's ring current. (from Hamilton et al., 2013)

\subsection{Contribution of the CRAND source}

Four missions visited the Kronian magnetosphere (Pioneer 11, the Voyagers and Cassini) and reported the existence of a significant population of energetic charged particles $(>200 \mathrm{keV})$ in the radiation belts of Saturn (Cooper and Simpson, 1980; Krimigis and Armstrong, 1982; Krimigis et al. (2005)). The Saturnian moons Tethys and Dione prevent inward radial transport of energetic ions and isolate inner ion radiation belts from the middle and outer magnetosphere (Roussos et al. (2008), Roussos et al. (2011), Paranicas et al. (2008)). Therefore the MeV ion radiation belts exist permanently inside of the $\mathrm{L}$-shell of Tethys $(\mathrm{L}=4.89)$ and remain unchangeable during the interplanetary events, which cause large perturbations in the magnetosphere beyond the Tethys orbit. Figure 27 represents the general structure of Saturn's radiation belts using the Cassini MIMI/LEMMS data. 


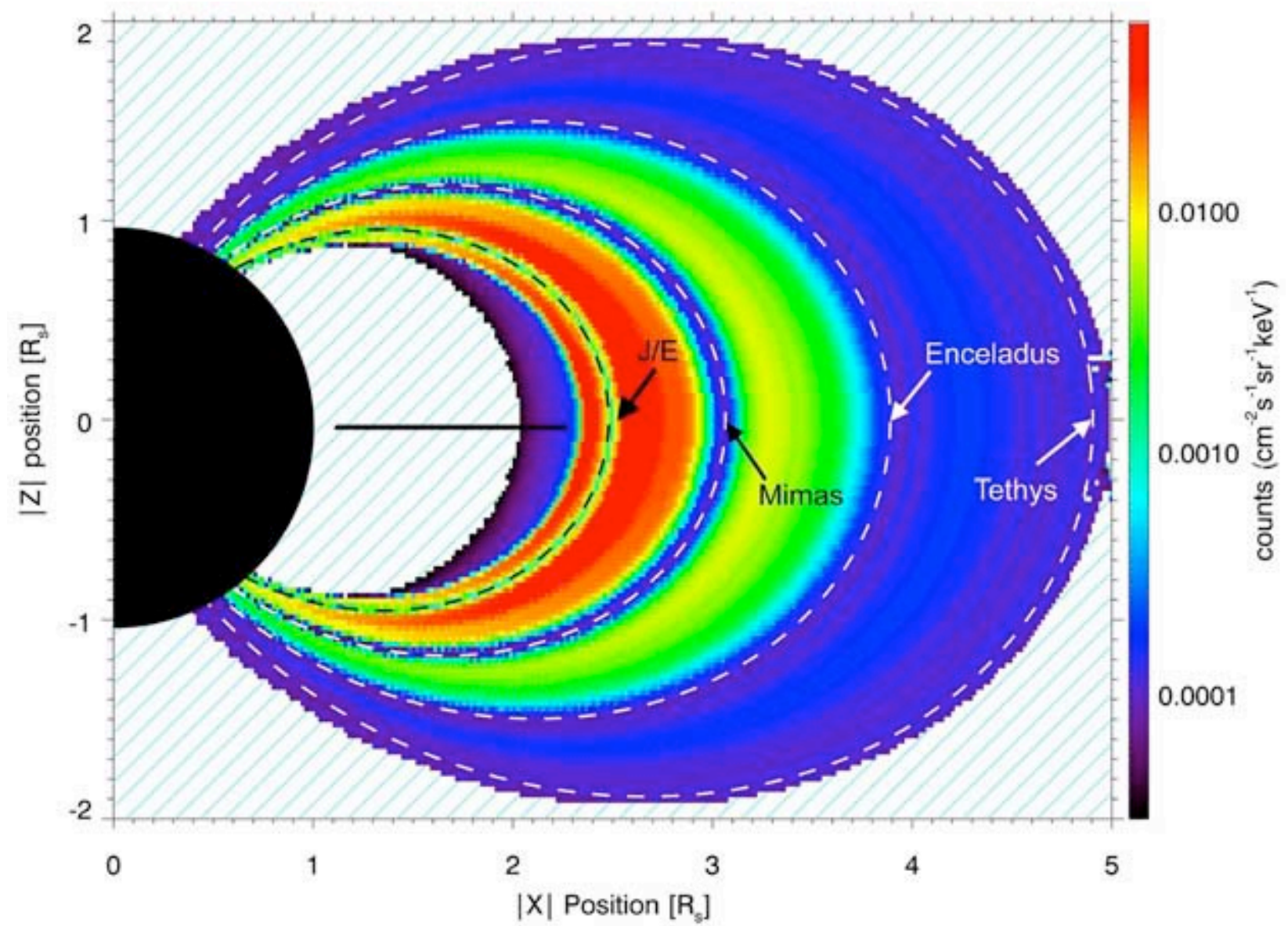

Figure 27. Differential flux map of the stable belts inside Tethys' L-shell of the $25-60 \mathrm{MeV} / \mathrm{nuc}$ ions, based on LEMMS data from 36 orbits. The L-shells of the various moons are indicated. The partial flux dropout at the shell of the G-ring is also visible. Hatched regions above the main rings have particle flux lower or equal to that of the color bar. The radiation belt inside the D-ring (Krimigis et al., 2005) is not presented here. (from Roussos et al., 2008)

At the L-shells of the inner moons Janus, Epimetheus, Mimas and Enceladus the energetic particle observations show clear depletions (Fillius and Ip, 1980; Krimigis and Armstrong, 1982; Simpson et al., 1980; Vogt et al., 1982) due to the absorption effect. Figure 28 demonstrates this feature. As it was described by Roussos et al. (2008) and Paranicas et al. (2008), this indicates that the sources for the energetic component of the inner radiation belt cannot originate from the outer magnetosphere. 


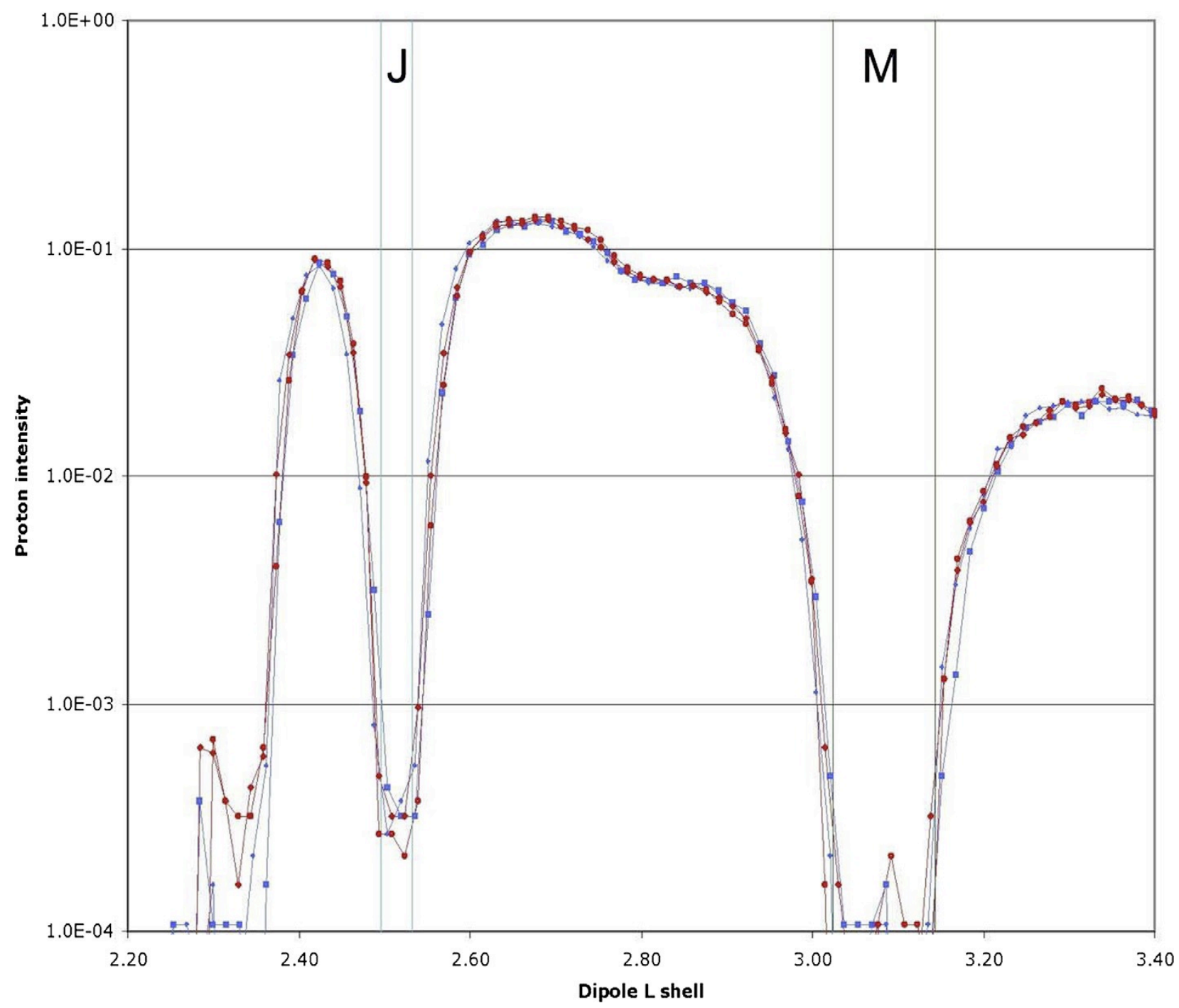

Figure 28. Proton intensity (protons per $\mathrm{cm} 2 \mathrm{~s} \mathrm{sr} \mathrm{keV)} \mathrm{as} \mathrm{a} \mathrm{function} \mathrm{of} \mathrm{L} \mathrm{shell} \mathrm{during} \mathrm{SOI.} \mathrm{To} \mathrm{compute} \mathrm{the} \mathrm{dipole} \mathrm{L}$ shell of the spacecraft and satellites, an offset dipole is used with moment along Saturn's spin axis. We plot (as separate curves) only those points corresponding to equatorial pitch angles of $25^{-35^{\circ}}$ and $145-155^{\circ}$. Furthermore, we have indicated which points were taken during the inbound (blue) and outbound (red) portion of the trajectory. Positions of the Satellites Janus $(\mathrm{L} \sim 2.5)$ and Mimas $(\mathrm{L} \sim 3.1)$ are shown as sweeping corridors. (from Paranicas et al. (2008)).

The possible mechanism responsible for populating the energetic ionic belts is then the CRAND process. Galactic cosmic rays with energies exceeding the cut-off rigidities required to reach the planetary atmosphere and/or the rings enter the magnetosphere, interact with the planetary atmosphere, rings, E ring and the extended neutral gas cloud, and create cascades of particles, partly at much lower energies, including neutrons, protons and other particles such as photons, electrons, pions, and various antiparticles (Kollmann et al. (2012)). Secondary charged particles will be almost immediately lost after bouncing back along the field lines, to the location where they were produced. However, neutrons can travel away from their production region (since they are not bound by the magnetic field). The small fraction of those that will beta-decay within the strong dipole region, will populate the radiation belts with energetic electrons (mostly below 1 
$\mathrm{MeV}$ ) and protons. This mechanism was discussed by several authors (Cooper and Simpson, 1980; Fillius and McIlwain, 1980; Van Allen et al., 1980; Blake et al., 1983; Cooper, 1983; Cooper et al., 1985; Randall et al., 1994) and Figure 29 illustrates it. Blake et al. (1983) proved theoretically that the high-energy component of the radiation belts originates from CRAND. The latest observations by Cassini confirmed this suggestion (Roussos et al. (2011)). Kollmann et al. (2012) specify that atmospheric CRAND is the central process initially providing the protons (from $500 \mathrm{keV}$ up to $40 \mathrm{MeV}$ ) and CRAND from the Main Rings contributes to some extent to the population, but only for $>10 \mathrm{MeV}$, while other possibilities to supply the belts and exchange particles between them, as diffusion and injections from outside the belts, or stripping of ENA's, can be excluded.

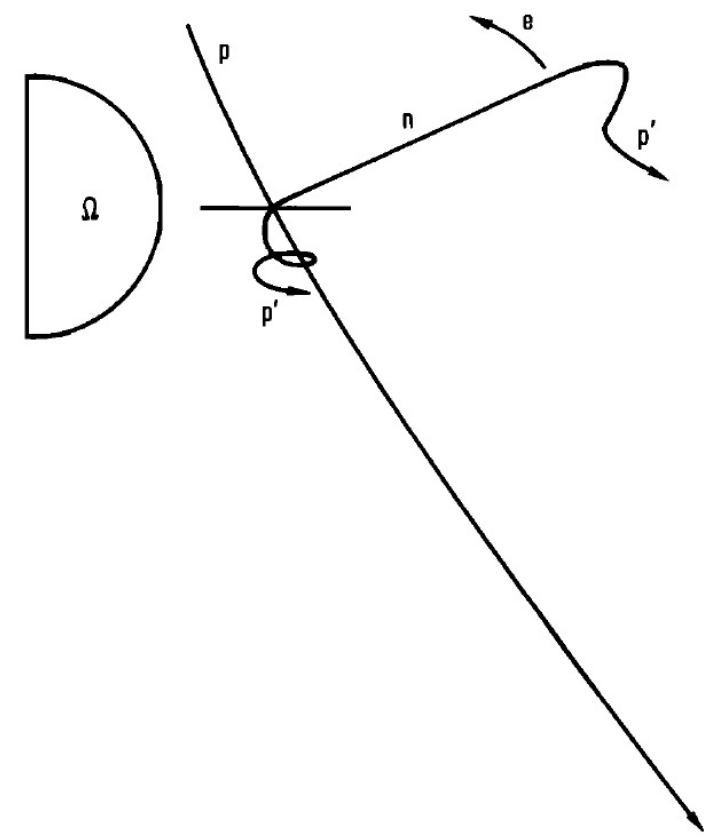

Figure 29. The CRAND process in the Saturnian system is illustrated schematically. An incoming cosmic ray proton comes from above, passes through a ring particle, and continues to infinity. A nuclear interaction creates a secondary proton and neutron. The proton is trapped in the magnetic field of Saturn and is removed within a few bounces by repeated passages through the rings. More neutrons are created. The first neutron decays in flight near Saturn injecting an energetic proton and electron into the Saturnian magnetosphere. (from Blake et al. 1983)

CRAND cannot be responsible for the presence of heavier species that have been detected in the belts (Armstrong et al., 2009). However, given that heavier species are distributed in the same way as $\mathrm{MeV}$ protons, their origin should involve also a stable, external cosmic ray source, without excluding also Anomalous Cosmic Rays.

Another interesting feature of the energetic radiation belt is that the MIMILLEMMS instrument onboard Cassini detected a weak intensification of the trapped proton component during solar minimum, which demonstrated the dependence of the proton source on the GCR access to the Saturnian system (Roussos et al., 2011).

\subsection{Summary}

Suprathermal ion abundances should broadly reflect the source rates of the various species, although some acceleration processes can change abundances compared to the low energy 
plasma as can differences in loss rates. The similar radial profiles of most suprathermal species in the $10-20 \mathrm{R}_{\mathrm{S}}$ range, indicate they have probably undergone similar acceleration processes. Differences within $10 \mathrm{R}_{\mathrm{S}}$ are largely due to different charge exchange and other loss cross sections in the neutral cloud originating from Enceladus. Long term factor 2 constancy in the PNDs of the major suprathermal species from 2004 to 2012 indicates relatively stable plasma source rates. The strongest plasma sources are local as is evident from the low abundance of $\mathrm{He}^{++}$and $\mathrm{He}^{+}$, which originate from outside the magnetosphere. The very high $\mathrm{H}^{+} / \mathrm{He}^{++}$ratio indicates that only about $16 \%$ of the magnetospheric protons come from the solar wind, with sizeable uncertainty. Trace species such as $\mathrm{H}_{3}{ }^{+}$and $\mathrm{O}_{2}{ }^{+}$indicate that Saturn's ionosphere and rings also contribute some plasma to the magnetosphere. The seasonal variation of the $\mathrm{O}_{2}^{+} / \mathrm{W}^{+}$ ratio confirms that the intensity of ring illumination largely controls the $\mathrm{O}_{2}{ }^{+}$source rate. Trace species $\mathrm{C}^{+}$and $\mathrm{N}^{+}$probably arise from Enceladus, although Titan contributions have not been completely ruled out.

In the energetic ion range, at the top of the energy range, the CRAND mechanism is likely to produce a strong inner proton belt, as in other planetary magnetospheres. This process has been confirmed by Cassini studies. Recent studies and modeling results show that CRAND is a sufficient source process to populate the observed energetic protons flux in the inner magnetosphere of Saturn. In particular, for the energetic particles with energies of hundreds keV - tens $\mathrm{MeV}$, atmospheric CRAND is most likely the central source process, while the CRAND from Main Rings plays an important role for producing protons with energies above $10 \mathrm{MeV}$ and thereby amplifies atmospheric CRAND for this energy range.

\begin{tabular}{|l|l|l|}
\hline Species & Relative to $\mathbf{H}^{+}$ & Relative to Total \\
\hline $\begin{array}{l}\mathrm{W}^{+} \\
\mathrm{O}^{+} \quad 53 \% \\
\mathrm{OH}^{+} 22 \% \\
\mathrm{H}_{2} \mathrm{O}^{+} 22 \% \\
\mathrm{H}_{3} \mathrm{O}^{+} 2.8 \%\end{array}$ & 2.1 & 0.61 \\
\hline $\mathrm{H}^{+}$ & & \\
\cline { 2 - 3 } & & \\
\cline { 2 - 3 } $\mathrm{H}_{2}^{+}$ & $\equiv 1$ & \\
\hline $\mathrm{O}_{2}^{+}$ & 0.17 & 0.30 \\
\hline $\mathrm{O}^{++}$ & 0.037 & 0.050 \\
\hline $\mathrm{He}^{+}$ & 0.032 & 0.011 \\
\hline $\mathrm{N}^{+}$ & 0.018 & 0.0096 \\
\hline $\mathrm{C}^{+}$ & 0.016 & 0.0054 \\
\hline $28 \mathrm{M}^{+}$ & 0.011 & 0.0048 \\
\hline $\mathrm{H}_{3}^{+}$ & 0.0087 & 0.0026 \\
\hline $\mathrm{He}^{++}$ & 0.0072 & 0.0021 \\
\hline & 0.0029 & 0.00086 \\
\hline
\end{tabular}


Table 1 : Average suprathermal ion abundances in Saturn's magnetosphere (dipole L = 7-16, near equatorial $\left( \pm 10^{\circ}\right)$, late 2004 to end of 2010). Abundances reflect relative partial number densities over the stated energy ranges. The energy range for $\mathrm{W}^{+}, \mathrm{H}^{+}, \mathrm{H}_{2}^{+}, \mathrm{O}^{++}, \mathrm{He}^{+}, \mathrm{He}^{++}$is 27 $220 \mathrm{keV} / \mathrm{e}$ (DiFabio 2012). The energy ranges for some of the rarer or less well-resolved species are more restrictive: $83-167 \mathrm{keV} / \mathrm{e}$ for $\mathrm{O}_{2}{ }^{+},{ }^{28} \mathrm{M}^{+}\left(\mathrm{N}_{2}{ }^{+}\right.$and/or $\mathrm{CO}^{+}$) (Christon et al., 2013); $127-$ $220 \mathrm{keV} / \mathrm{e}$ for $\mathrm{N}^{+}, \mathrm{C}+\left(\right.$ DiFabio, 2012); 36-167 keV/e for $\mathrm{H}_{3}{ }^{+}$(Hamilton et al., 2013). The makeup of the water group ions $\mathrm{W}^{+}$was determined at 94-97 keV/e (DiFabio, 2012).

\section{Transport and acceleration processes and related losses}

\subsection{Interchange signatures for ions and electrons}

The inner regions of Saturn's magnetosphere are supplied with plasma produced by photoionization, charge exchange and electron impact ionization of a cloud of neutral water molecules (Shemansky et al., 1993, Esposito et al., 2005, Young et al., 2005, Perry et al., 2010) which dominate the particle density in these regions between $\sim 3$ and 8 Saturn radii, Rs. As developed in detail in section 2, Cassini has shown that these neutrals emanate from Enceladus itself. This followed the discovery of a dynamic atmosphere (Dougherty et al., 2006) and vast plumes of neutrals (Waite et al., 2006), plasma (Tokar et al., 2006, 2009), water clusters (Coates et al., 2010) and neutral and charged dust particles (Spahn et al., 2006, Jones et al., 2009) emanating from tiger stripe features on the surface (Porco et al., 2006).

The first results from the CAPS instrument in Saturn's magnetosphere showed that the composition is dominated by the water group. In addition, the density of plasma is much less than that of neutrals (Young et al., 2005). The almost co-rotating inner magnetosphere, which includes hydrogen ions likely to be mainly from Saturn's ionosphere, is dominated by waterbased neutrals $(\mathrm{O}, \mathrm{OH})$. Enceladus, supplemented by the rings and the associated neutrals, populates the outer magnetosphere as well (Smith et al., 2009, Thomsen et al., 2010, Arridge et al., 2011). Some of the remarkably complex chemistry at Titan appears to involve particles, oxygen in particular, originally from Enceladus (Coates et al., 2007b, Sittler et al., 2009). The electron populations in the inner magnetosphere, as well as the water-rich composition, show remarkable structure and dynamics (e.g., Figure 30, from Young et al., 2005). There is a cold component, the upper energy of which appears to be controlled by the proton corotation speed (Young et al., 2005) and a hot component, which appears separate and is supplemented in the inner magnetosphere by remarkable injections, periodicities and interchange events. Further analysis of the hot and cold populations has been provided by Rymer et al. (2007) and Schippers et al. (2009). Interchange is a process known from Jupiter's rapidly spinning magnetosphere, where the mainly cold, dense, corotating plasma in the inner magnetosphere interchanges with hot, rare plasma in the outer magnetosphere, driven by radial transport. The resulting structure resembles 'fingers' interleaving the two populations (e.g. Hill et al., 2005; Liu et al., 2010; see Figures 31 and 32). Following the interchange event, the ions and electrons undergo gradient/curvature drift dispersion. Cassini observations revealed this structure in both ions and 
electrons (Hill et al., 2005) together with its magnetic counterpart (André et al., 2007; Leisner et al., 2005).

Electrons produced in Saturn's inner magnetosphere circulate with a combination of outward and inward motions driven by the centrifugal interchange instability, and azimuthal motion through gradient and curvature drifts (Rymer et al., 2008). Cool $(<100 \mathrm{eV})$ electrons produced inside L $\sim 12$ move slowly outward. To balance the outflowing flux, inward transport occurs in small scale injection events. Electrons in these inwardly moving flux tubes are heated adiabatically to energies greater than $100 \mathrm{eV}$ and their pitch angle distributions evolve from isotropic to "pancake" (peaked at $90^{\circ}$, see Figure 33). The hot electron component in Saturn's magnetosphere is thus formed by the drift and dispersion of electrons from these small-scale inflow channels (Hill et al., 2005; Burch et al., 2005; Rymer et al., 2008).

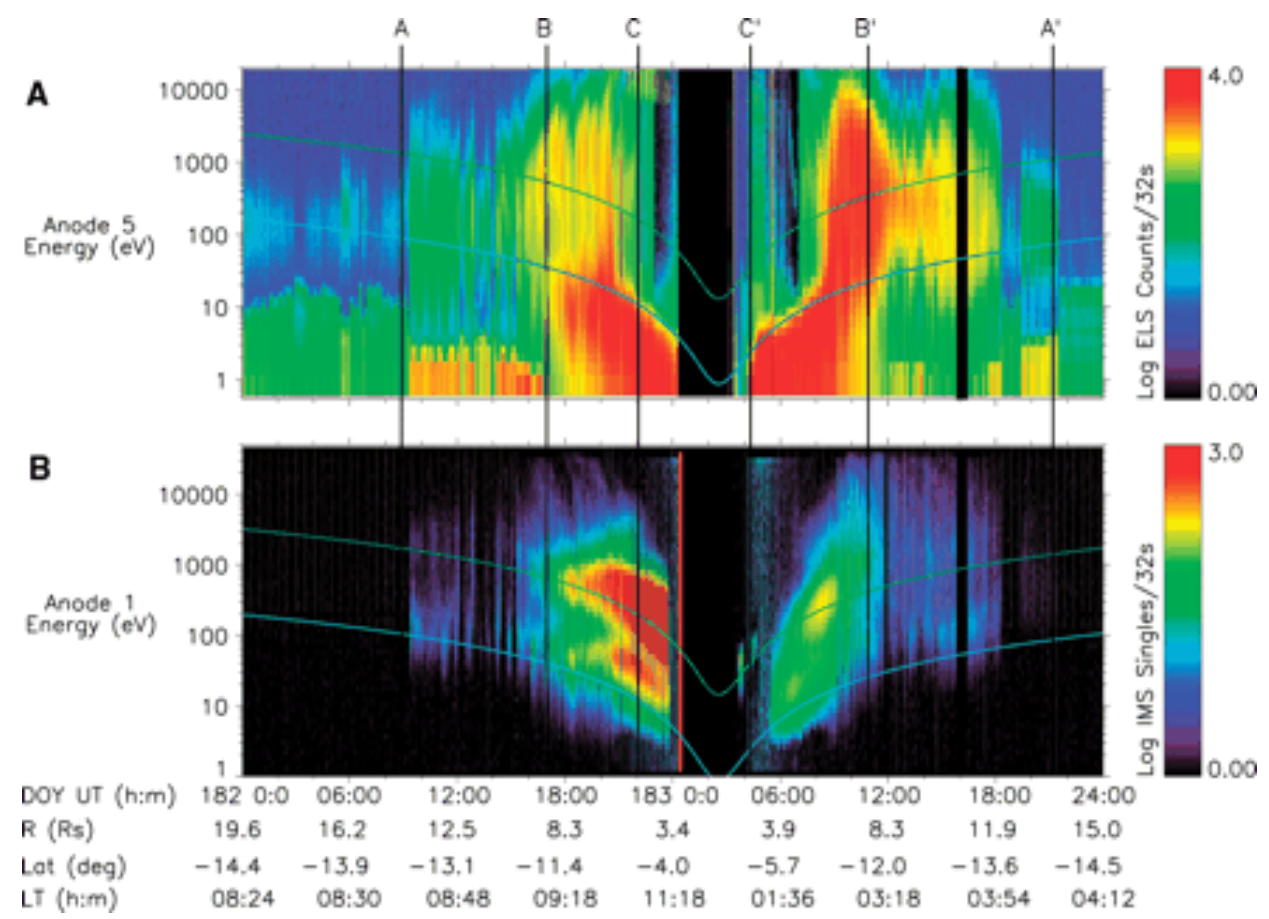

Figure 30 : Electron (upper panel) and ion (lower panel) spectrograms for two days of the Saturn insertion orbit, with proton (lower trace) and $\mathrm{W}+$ (upper trace) energies overlaid. (from Young et al. 2005) 


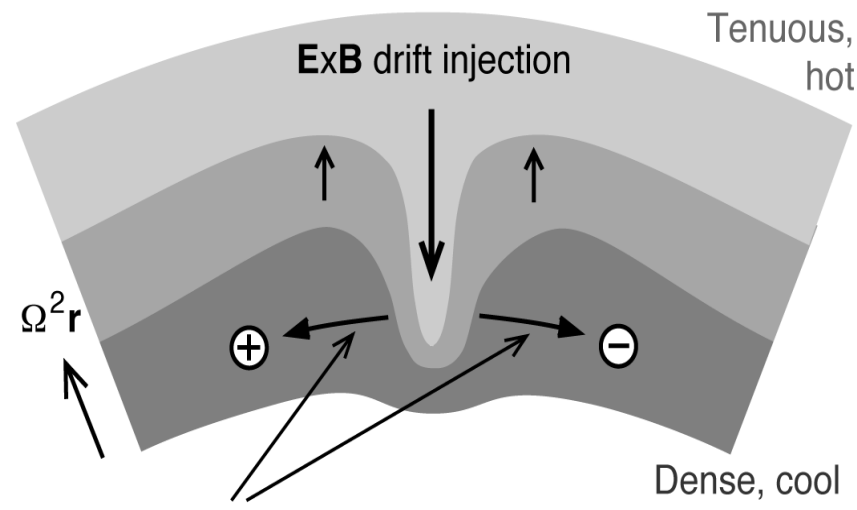

Gradient/curvature drift dispersion

Figure 31 : Schematic of interchange event (from Hill et al., 2005)
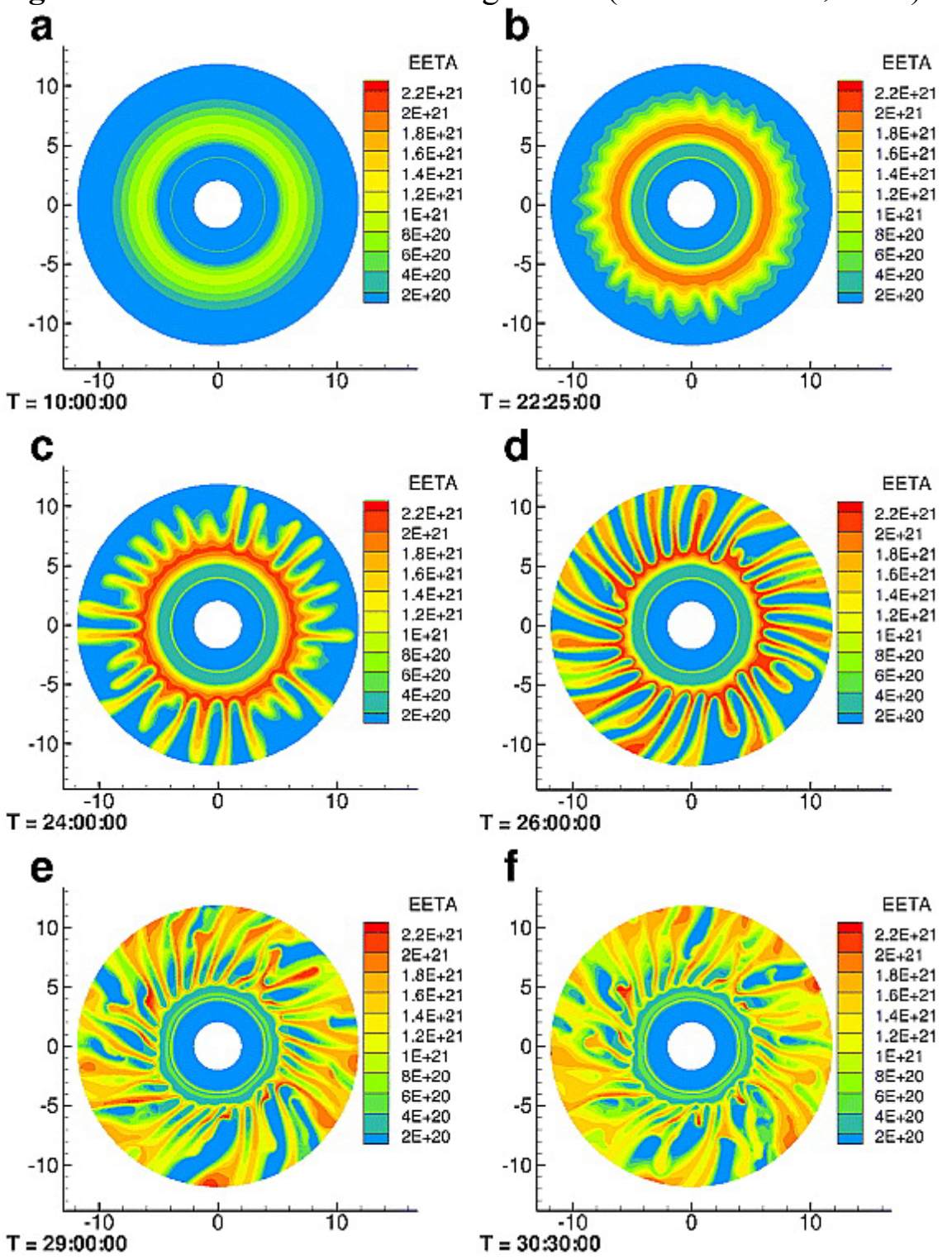
Figure 32 : Simulation showing interchange 'fingers' (from Liu et al., 2010).

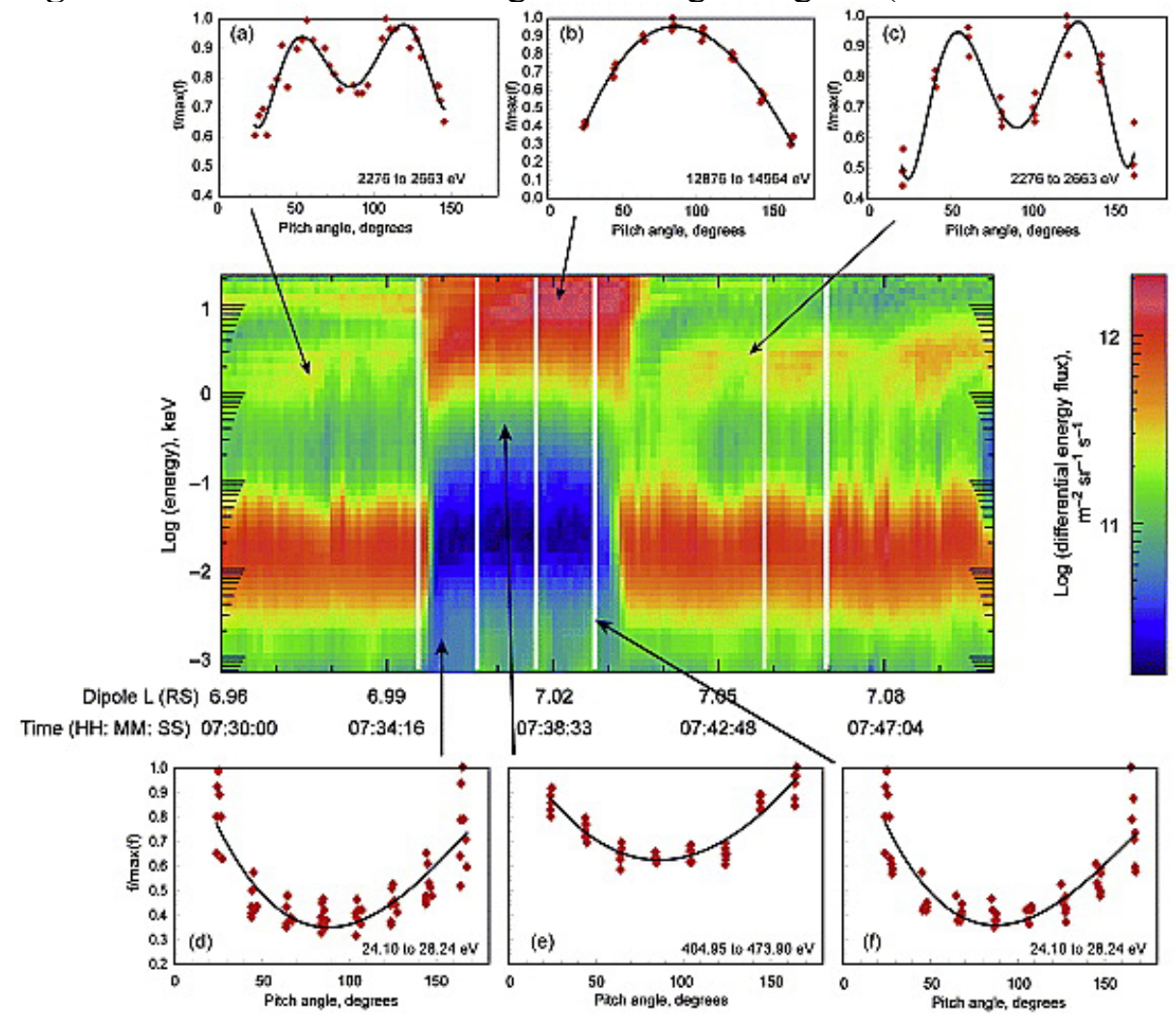

Figure 33 : Electron injection event observed on 30 October 2005 (DOY 303). The insets show electron phase space density versus pitch angle distributions derived from ELS data at the times and energies indicated by the arrows along with polynomial fits to the data (from Rymer et al., 2008)

\subsection{Reconnection/Plasmoids}

Magnetotail reconnection is a process which can allow large amounts of material to be broken off on the nightside and lost from the magnetosphere. The first chance to search for evidence of this process came with the arrival of Cassini in 2004, and particularly with the deepest tail orbits in 2006 when the spacecraft reached downtail distances of $68 \mathrm{R}_{\mathrm{S}}\left(1 \mathrm{R}_{\mathrm{S}}=\right.$ $60268 \mathrm{~km}$ ). Figure 34 shows the trajectory of Cassini during this time, with the timings of observed reconnection events marked. Events are identified initially by bipolar changes in the $\mathrm{B}_{\theta}$ (north-south) component of the field, and, where plasma data are available, by concurrent changes in local plasma properties and plasma flow direction. The events displayed in Figure 34 include a total of 69 plasmoids, 17 travelling compression regions (TCRs) and 13 planetwardmoving events. The direction of motion is inferred in the first instance from the sign of the change in $\mathrm{B}_{\theta}$.

The properties of plasmoids and their effect on the local environment (in the form of TCRs) (e.g. Jackman et al., 2008b; 2009a; 2009b) and the global magnetosphere (in terms of changing plasma flows, flux closure, mass removal) (McAndrews et al., 2009; Jackman et al., 2011; Jia et al., 2012a; Thomsen et al., 2013) have been explored by a number of authors since the observation of the first three cases with magnetic field (Jackman et al., 2007) and plasma data (Hill et al., 2008). We refer the reader to these papers and to the reviews of Thomsen (2013) and 
Jackman (2014) for further comprehensive description of Saturn's magnetotail dynamics. For a comprehensive review of the dynamics of Saturn's magnetotail, compared and contrasted with Mercury, Earth and Jupiter, we refer to Jackman et al. (2014a). An example of two plasmoids and two TCRs is shown in Figure 35. The interior structure of plasmoids is found to be primarily loop-like, as opposed to helical twisted flux ropes commonly seen in the Earth's magnetosphere. Some flux ropes are observed, but their relative scarcity may mean that the large-scale structure of Saturn's magnetotail field is less sheared than at other planets (Jackman et al., 2014b).

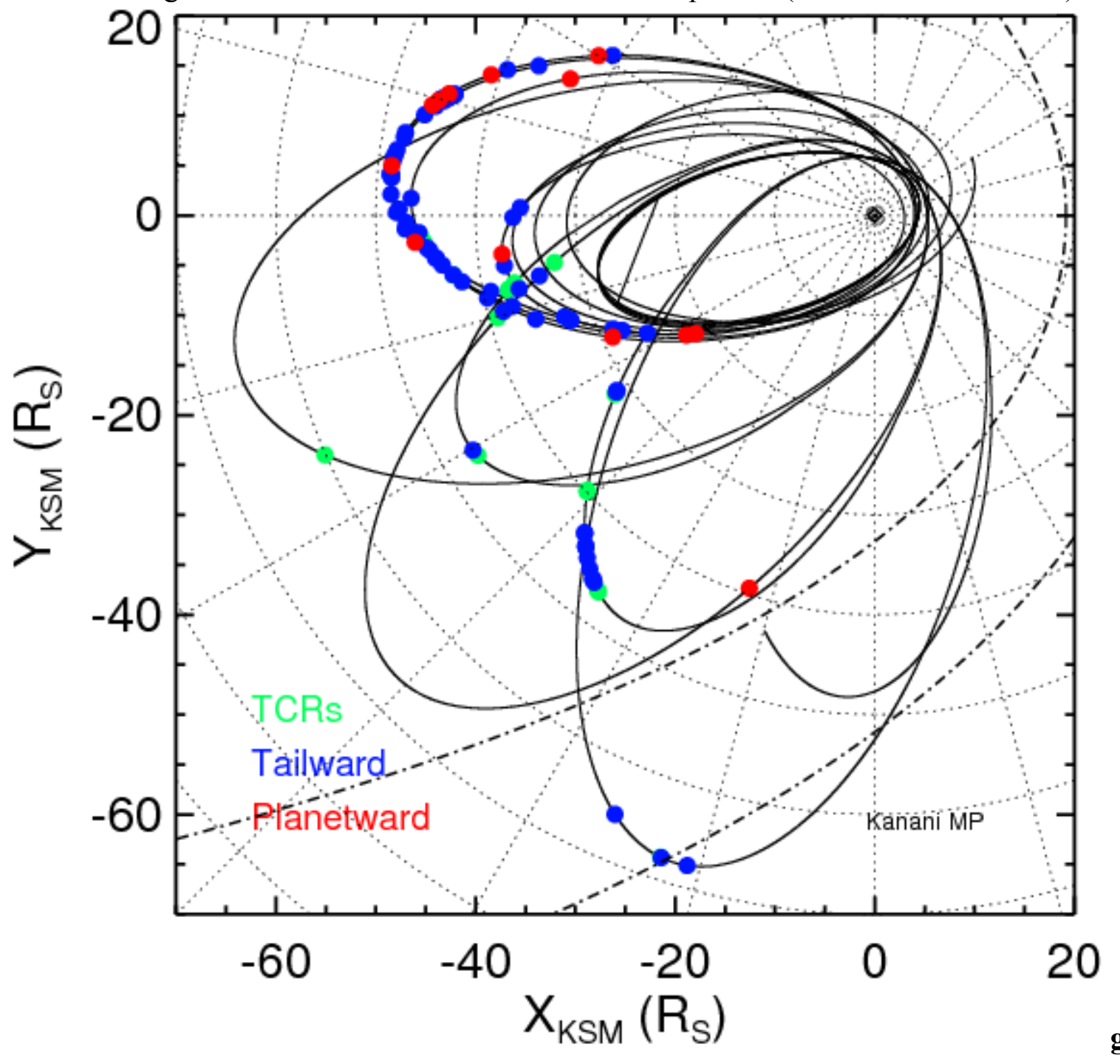

Figure 34: X-Y projection of the Cassini trajectory for 2006 day 18-291 in the Kronocentric Solar Magnetospheric (KSM) co-ordinate system. KSM is the kronian analogue of GSM where the $\mathrm{X}$ axis coincides with the direction to the Sun, the $\mathrm{XZ}$ plane contains the planetary dipole axis, and the $\mathrm{Y}$ component is azimuthal, positive toward dusk. Blue, red and green dots show the location of tailward and planetward-moving structures, and TCRs respectively. The Kanani et al. [2010] model magnetopause is overplotted for solar wind dynamic pressures of 0.1 and $0.01 \mathrm{nPa}$. (from Jackman et al., 2014b). 


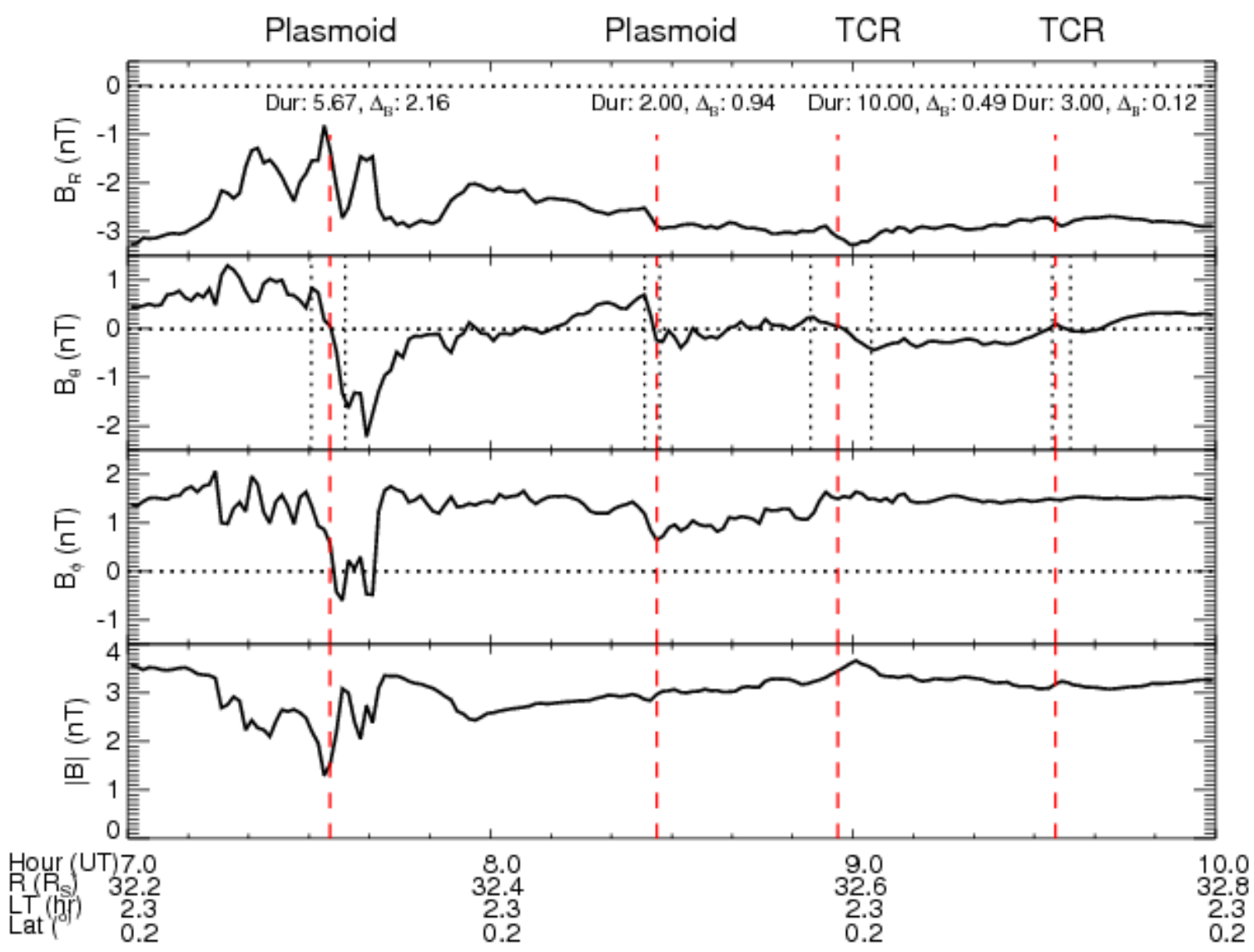

Figure 35: Cassini magnetic field data in KRTP co-ordinates for 2006 day 60 07:00-10:00, where the radial component $\left(\mathrm{B}_{\mathrm{r}}\right)$ is positive outward from Saturn, the theta component $\left(\mathrm{B}_{\theta}\right)$ is positive southward, and the azimuthal component $\left(\mathrm{B}_{\varphi}\right)$ is positive in the direction of planetary corotation. The positions of two plasmoids and two TCRs are marked with vertical lines, and the amplitude and duration in minutes of the signatures are listed in the top panel. The plasmoids are identified by northward turnings of the field, while the TCRs are identified by localized compressions in the total magnetic field strength, and small northward turnings. It is inferred that the spacecraft penetrated most deeply into the first plasmoid, evidenced by the local decrease in $|B|$. (from Jackman et al., 2014b).

\subsection{Field-aligned acceleration and current generation}

\section{Field-aligned currents}

Electric currents commonly arise in planetary magnetospheres as a result of plasma flow shears, pressure gradients, or inertial stresses (Baumjohann et al., 2010). These currents play a crucial role in magnetosphere-ionosphere coupling, and Saturn's magnetosphere is no exception. Like its giant planet sibling, Jupiter, Saturn also is a rapid rotator and contains significant internal plasma sources supplied by its satellites and rings with Enceladus being the dominant contributor. Both the pick-up of newly produced plasma from the internal sources and the subsequent outward transport of the magnetospheric plasma tend to slow down the local flow, leading to the lag of plasma with respect to rigid corotation that consequently causes the 
magnetic field lines to bend backward. Corresponding to the bendback magnetic geometry is a radial current flowing through the equatorial plasma, which exerts a $\mathrm{JxB}$ force on the magnetospheric plasma that acts to accelerate it toward corotation. The radial, transverse current is then closed through field-aligned currents flowing between the ionosphere and the magnetosphere, forming an internally driven current system referred to as the corotation enforcement current (e.g., Hill, 1979). The corotation enforcement current system is inherent in a rapidly rotating magnetosphere with a strong internal plasma source that is ultimately responsible for the momentum transfer between the planet and the mass-loaded magnetosphere. It is generally believed that the enforcement current system is responsible for the generation of the main auroral oval at Jupiter. While a similar situation might be expected for Saturn, it is unlikely to be the case because the region of corotation breakdown observed in Saturn's inner magnetosphere maps to too low latitudes compared to where the main oval is typically observed, and the upward field-aligned currents associated with the enforcement current system are too weak and do not appear to require the significant parallel acceleration which is needed for producing the observed auroral emissions (e.g., Cowley et al., 2004, 2008).

Instead, Saturn's main auroral oval has been suggested to be associated with processes occurring in the middle or outer magnetosphere. For instance, Sittler et al. (2006) proposed a model to explain Saturn's main auroral oval in which the source location of the oval maps to the outer edge of the plasma sheet located in the middle magnetosphere, where centrifugally driven interchange instability may produce significant particle acceleration and precipitation into the ionosphere leading to auroral emissions. Another model, proposed by Cowley and Bunce (2003) and Cowley et al. (2004), associated Saturn's main oval with the open-closed field line boundary in the polar ionosphere. Equatorward of the open-closed field line boundary are closed field lines mapped to the outer magnetosphere that contain plasma moving in the corotation direction at a fraction of the rigid corotation speed, whereas poleward of this boundary are open field lines whose motion is governed in combination by the solar wind from above and the ionosphere from below. The flow shear between the open and closed field lines implies the existence of a layer of upward field-aligned currents flowing near the boundary that are likely to require field-aligned electric fields to develop, in which case electrons are accelerated into the ionosphere producing aurora. In-situ observations of the magnetospheric conditions combined with remote observations of the aurora provide the best opportunity to test these scenarios. For example, Bunce et al. (2008) combined the HST observations of the aurora with simultaneous measurements of the particles and fields from one of Cassini's high-latitude passes through the dayside magnetosphere to show that the observed noon aurora lies close to the boundary separating the open and closed field lines. Talboys et al. (2011) later carried out a comprehensive survey of high-latitude field-aligned currents signatures using Cassini magnetic field data and compared their locations with the open-closed field line boundary inferred from particle data. The statistical results of Talboys et al.. (2011) indicated that the upward field-aligned currents are typically seen not right at the open-closed field boundary, as predicted by the Cowley et al., (2004) theoretical model, but rather in a region equatorward of the boundary, which presumably maps to closed field lines in the outer magnetosphere. While the discrepancy between the observations and the theoretical model remains to be understood through future work, possible factors, such as the inhomogeneity of ionospheric conductivities and the effect of the magnetospheric periodicities, have been proposed that may account for the difference (Talboys et al., 2011). 
Regardless of their source, field-aligned currents require current carriers and electrons are usually the primary carrier due to their mobility. For a given amount of current demanded by any magnetospheric process, if there are not sufficient electrons available to carry the required current, then field-aligned electric fields normally develop to accelerate the current-carrying electrons (see the chapter by Seki et al. in this issue). In regions of downward flowing currents, a field-aligned potential drop may develop that would accelerate electrons out of the ionosphere forming field-aligned electron beams. Evidence for such electron beams has been found in Saturn's high-latitude region. Cassini frequently observed upward propagating whistler-mode hiss emissions in the auroral zone (Gurnett et al., 2009), which are believed to be produced by upward moving electron beams associated with the downward flowing field-aligned currents (e.g., Kopf et al., 2010). In regions of upward flowing currents, parallel electric fields would accelerate electrons into the atmosphere/ionosphere where they can lead to significant magnetospheric consequences, such as the excitation of aurora, generation of radio emissions, and enhancement of ionospheric conductivity. For this reason, it is important to understand the acceleration process associated with field-aligned currents.

Parallel electric fields usually develop somewhere above the ionosphere; however, the exact location of the acceleration region depends on the electron distribution along the field lines. It has been suggested that the acceleration region at Saturn is likely to lie at $\sim 0.5$ Saturn radius above the ionosphere (Ray et al., 2013), which is below the lowest altitude that Cassini has thus far reached during its high latitude passes. In the absence of direct observations of the acceleration region, understanding of the acceleration process may rely on theoretical models developed for understanding similar processes occurring in other planetary magnetospheres, such as the current-voltage relation proposed by Knight (1973) for the terrestrial magnetosphere. However, the way in which the magnetospheric plasma is distributed in Saturn's magnetosphere is largely affected by the planetary rotation and the presence of strong internal plasma sources, a situation quite different from the terrestrial case. This aspect of the Saturnian system needs to be taken into account when considering the relationship between field-aligned current and fieldaligned potential drop.

For a rapidly rotating magnetosphere like that of Saturn, centrifugal effects play an important role in determining the plasma distribution within the magnetosphere. The centrifugal acceleration tends to push plasma radially outward and to stretch magnetic field lines, which leads to equatorial confinement of the magnetospheric plasma. For a multi-species plasma, as is the case for Saturn's magnetosphere that consists primarily of heavy water-group ions, protons and electrons, the plasma distribution along magnetic field lines is determined by the centrifugal force, the gravitational force and the force associated with the ambipolar electric field (e.g., Sittler et al., 2008). It has been found that the heavier, water-group ions are more strongly confined to the equator while the lighter species (e.g., protons and electrons) are distributed more broadly along the magnetic field lines (e.g., Thomsen et al., 2010). However, it is possible that a low electron density region exists somewhere at mid latitude where the sum of the gravitational and centrifugal potentials exhibits a local minimum. 
The latitudinal plasma distribution in Saturn's magnetosphere has important implications regarding the field-aligned current generation and associated particle acceleration. Ray et al. (2013) have used a one-dimensional Vlasov simulation to study Saturn's current-voltage relation taking into account the effect of centrifugal confinement of the magnetospheric plasma. They found that the relationship between the field-aligned potential drop and field-aligned current density derived from their simulations is essentially consistent with the prediction of the Knight [1973] kinetic theory. Their simulation results, however, emphasized the need of using plasma conditions at the top of the acceleration region, instead of those of the equatorial plasma sheet, in order to obtain an accurate estimate of the field-aligned potential drop.

\subsection{Planetary period oscillations and consequences}

Oscillations with periods close to the estimated rotation period of Saturn $(\sim 10.6 \mathrm{~h})$ have been detected in a multitude of magnetospheric parameters at Saturn, beginning with the initial detection in Voyager Planetary Radio Astronomy data of a strong modulation in the brightness of the Saturn Kilometric Radiation (SKR, a circularly polarised auroral radio emission with frequencies of tens to hundreds of kHz [Kaiser et al., 1980; 1981; Warwick et al., 1981; Desch and Kaiser, 1981; Desch, 1982]). Subsequent further studies using data obtained during the Pioneer and Voyager flybys showed corresponding modulations to be present in the magnetospheric plasma populations and external magnetic field [e.g. Carbary and Krimigis, 1982, Espinosa and Dougherty, 2000]. Detailed studies of modulations in the SKR and the magnetic field, from which quasi-continuous measurements of the oscillation parameters can be determined, have shown that the phase of the perturbations remains incredibly stable, with only slow drifts in period occurring on secular (seasonal) timescales [e.g. Galopeau and Lecacheux, 2000; Kurth et al., 2007, 2008; Andrews et al., 2008; Provan et al., 2009; Andrews et al., 2012]. We refer the reader to the recent review of this topic by Carbary and Mitchell [2013] for a more complete introduction, and instead only discuss here those aspects of this phenomenon of direct relevance to this chapter.

Recent discovery of a weaker, apparently independent modulation in the SKR originating in the northern hemisphere (i.e., the opposite hemisphere to that preferentially illuminated during both the Pioneer-Voyager and Cassini epochs), corroborated by measurements made in the magnetic field and related plasma populations, has shown the system to be significantly more complex than first thought [Gurnett et al., 2009, 2010; Andrews et al., 2010; Southwood, 2011; Provan et al., 2011]. Both atmospheric and magnetospheric sources have been proposed as possible origins of this system of large-scale, stable oscillations [Hill et al., 1981; Goldreich and Farmer, 2007; Gurnett et al., 2007; Southwood and Kivelson, 2007; Smith, 2011; Jia et al., 2012]. In Figure 36, taken from Gurnett et al. [2007], a so-called rotating convection model is depicted, in which a stable outflow of plasma originating from Enceladus is established through a twin-cell convection pattern, having a single outflow and a single inflow sector. Gurnett et al. [2007] provided evidence for a rotating modulation in the equatorial plasma density within $\sim 5 \mathrm{RS}$, while Burch et al. [2009] suggested that a corresponding systematic rotating modulation in the ion and electron count rates was present at larger radial distances, out to the magnetopause. However, the relative phasing between these apparent modulations in plasma density and the rotating magnetic field remains to be understood. Jia et al. (2012) and Jia and Kivelson (2012) have developed a magnetohydrodynamic simulation of the coupled magnetosphere-ionosphere 
system that captures a host of observed magnetospheric periodicities with considerable fidelity. In their model, rotating vortical flows in the upper atmosphere, through coupling to the ionosphere and the magnetosphere, drive field-aligned currents that periodically modulate the entire magnetosphere.

The dual nature of these periodicities (comprised of independent modulations linked to the two hemispheres) presents some difficulty in envisaging a purely magnetospheric origin of the phenomena. Nevertheless, all theoretical models of these phenomena contain rotating systems of field-aligned currents with an $\mathrm{m}=1$ azimuthal wavenumber, closing to some extent through both the ionosphere and equatorial magnetosphere, so as to account for the observed modulations in the SKR and magnetic field. A schematic of one such system of currents, and the implied magnetic field perturbations, is illustrated in Figure 37 . The presence of equatorial closure currents is required as a consequence of the simultaneous presence of independent northern and southern modulations in both the SKR and magnetic field, and the high-degree of apparent 'purity' in these modulations.

The extent to which this phenomenon drives, or is driven by, dynamical processes in the magnetospheric plasma populations remains to be demonstrated. Chen and Hill [2008] studied so-called 'injection events', in which plasma depleted flux tubes are interchanged with denser ones, a process by which transient radial plasma transport is achieved. Such injection events are regularly detected at radial distances of 5-10 RS. In their statistical survey of Cassini CAPS and MIMI data, Chen and Hill [2008] found no strong evidence for a periodicity to these injection events, or indeed any persistent organisation by the rotating phase of the SKR modulation. Subsequent analysis of injection events detected in Cassini RPWS data has presented strong evidence that, within a restricted range of local times near midnight, the occurrence rate of injection events is indeed well organised by the phase of the SKR emission, specifically that originating from the hemisphere that is in polar night [Kennelly et al., 2013].

Meanwhile, the possible relationship between observations of tailward-moving plasmoids at larger radial distances and the magnetospheric period oscillations was studied by Jackman et al. [2009a,b]. In particular, Jackman et al. [2009b] found that while the repetition time between the losses of plasmoids into the magnetotail was likely much longer than the rotation period, the release of these plasmoids was nevertheless reasonably well ordered by the phase of the SKR, with plasmoids observed more frequently during a 'preferential' phase sector.

Many open questions remain regarding the relationships between the observed modulations in the SKR, magnetic field, and plasma populations. It is likely that the magnitude of the quasisteady state convection velocity throughout the equatorial magnetosphere is sufficiently small (with respect to the bulk sub-corotational velocity) that direct detection of this systematic perturbation is essentially impossible with the available data. 

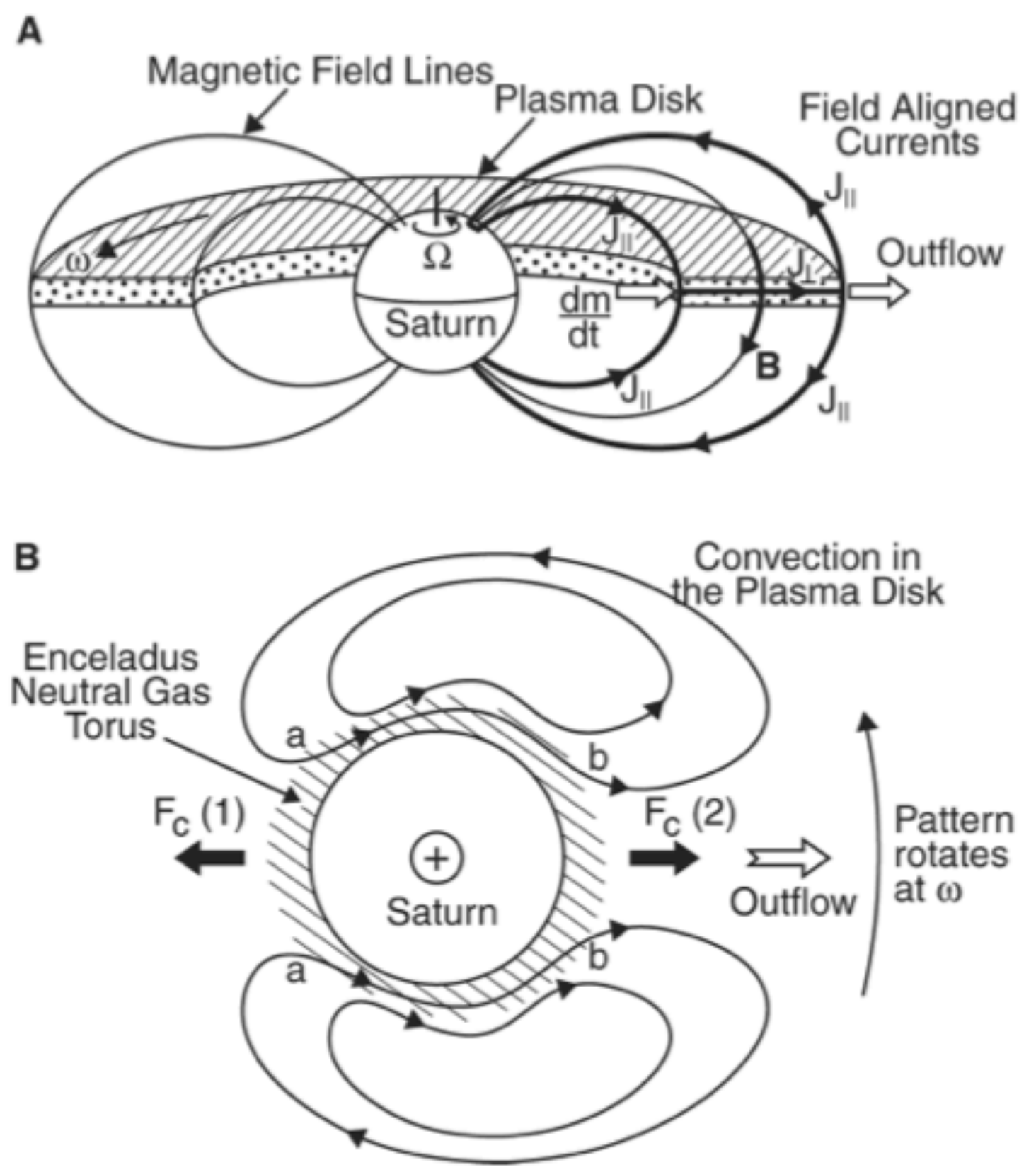

Figure 36 : Sketches of the rotating currents and associated plasma circulation streamlines within the rotating twin-cell convection pattern model proposed for Saturn. $\Omega$ is the angular rotation rate of Saturn, while $\omega$ is the corresponding rotation rate of the magnetospheric plasma. (from Gurnett et al., 2007). 

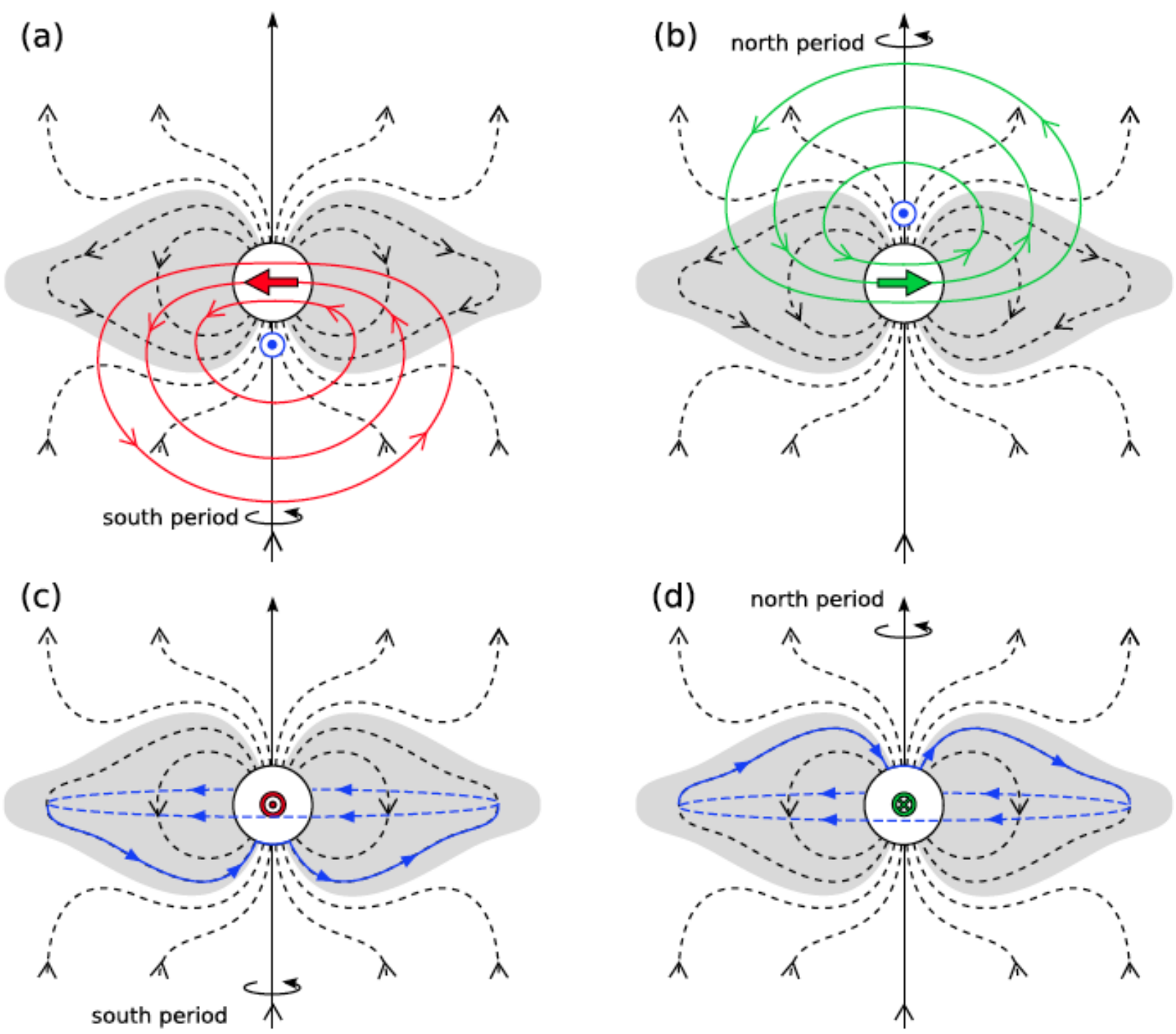

Figure 37: Sketches of the form of the oscillating magnetic fields deduced from Cassini observations, and the implied rotating current systems. Panels (a) and (b) show the rotating magnetic field signatures of the southern and northern systems as red and green solid lines, respectively, superposed on an illustrative sketch of the static (symmetric) planetary background field. These patterns then rotate in the same sense but with subtly differing periodicities associated with the SKR modulation in the corresponding hemisphere. The rotating current systems implied by these field perturbations are sketched in panels (c) and (d) by the blue solid lines, as viewed in a plane orthogonal to panels (a) and (b). (from Andrews et al., 2010).

\section{Summary, open questions and prospects for future studies}

Saturn's magnetosphere appears, in the light of Cassini and previous missions, to display a variety of plasma sources, and these sources interplay with a host of dynamical phenomena to produce a large set of spatial structures and temporal behaviors. This chapter has illustrated this diversity of phenomena. 
The first dominant feature of Saturn's magnetosphere examined from the viewpoint of its plasma sources is, just as for Jupiter, the dominance of one satellite source: to everybody's surprise, Cassini has revealed that the tiny icy satellite Enceladus and its southern hemisphere "tiger stripes" are the source of intense jets of water, called the Enceladus plumes, which provide the dominant source of water molecules for all the magnetosphere. This neutral water cloud spreads throughout the magnetosphere, in turn providing a source of plasma via a variety of ionization phenomena (e. g. UV photodissociation, UV and electron impact ionization, charge exchange). This source of plasma produces an ion torus which culminates somewhere outside the orbit of Enceladus and extends on either side of it. It is dragged into corotation via its coupling to Saturn's magnetic field and ionosphere/thermosphere.

One of the unique characteristics of this Enceladus ionized cloud system is that, near the location of Enceladus, the interaction of the charged particles with the water ice dust creates what one calls a "dusty plasma". So, Saturn is a unique place to study the behavior of this particular state of matter. Near the location of Enceladus, the dusty plasma indeed modifies the flow speeds of the plasma and the geometry of its interaction with the satellite.

Titan, which was suspected in the pre-Cassini years to be a major source of plasma for the magnetosphere, is in fact a minor source compared to Enceladus. It displays a cometary-type interaction with Saturn's corotating plasma, and is a limited source of both neutral and ionized particles. Neutral particle escape comes from the expanding exosphere of Titan, mainly $\mathrm{H}_{2}$, and to a lesser degree $\mathrm{N}_{2}$ and $\mathrm{CH}_{4}$. Ion escape results from the plasma interaction of Titan's ionosphere with its induced magnetosphere and the kronian plasma. Ions of ionospheric origin, such as $\mathrm{CH} 5+, \mathrm{C} 2 \mathrm{H} 5+$ and $\mathrm{HCNH}+$ leave the Titan environment and feed the magnetosphere in the vicinity of Titan's orbit, however this process is strongly disturbed by the interaction with the solar wind on the dayside of the Titan torus. Overall, the Titan interaction is interesting in itself, but provides only a minor source for the kronian magnetospheric plasma.

As for all other planetary magnetospheres, the solar wind is a likely source of plasma for Saturn, but the relative importance of this source is not quantified with great accuracy yet. The reason for this is that the efficiency of the dynamic coupling of the solar wind to the magnetospheric cavity, which depends on IMF orientation, plasma beta and Mach number of the interaction, is not known with certainty. Rather, opposing views on this subject are expressed, and more work is needed, if possible with direct measurements of the dynamic parameters in the vicinity of the magnetopause. In any case, and once more, the solar wind should remain a minor source compared to Enceladus, less than 5\% of the total supply according to most estimates.

In addition to the direct examination of the primary plasma reservoirs which feed the magnetosphere, another way of looking at plasma sources is to monitor the higher energy particles, which populate the magnetosphere after having been accelerated from the source regions. This chapter presents a comprehensive study of the distribution of these suprathermal and energetic particles, corroborating what we have learned from the examination of the main source regions.

The study of plasma sources cannot be separated from the one of the many dynamical phenomena acting on these sources, which tend to provide mechanisms for sources, transport and 
loss of plasma in each region, and which couple the different plasma reservoirs to the different dynamical modes of the magnetosphere as a whole. In this review chapter we gave an overview of some of these dynamical phenomena. In the tail, Cassini has unambiguously identified active magnetic reconnection producing plasmoids which, flowing downtail, evacuate plasma elements away from the magnetosphere and constitute an important plasma loss process. In the middle magnetosphere, flux tube interchange motions have been studied in considerable detail. These interchange motions contribute a lot to the radial redistribution of plasma. They are a key transport process for the magnetospheric plasma. On a more global scale, the kronian plasma is dynamically coupled throughout the magnetosphere to the magnetic field and high-latitude ionosphere. This coupling is the cause of the drag of the magnetospheric plasma into corotation. It operates via a current loop - the corotation-enforcement current - which connects the equatorial magnetosphere to the ionosphere. This process depends largely on the latitudinal distribution of the different plasma species, which it modifies in turn.

Finally, one of the strangest dynamical modes of Saturn's magnetosphere is the observed planetary period oscillations observed on most kronian magnetospheric parameters. The source of this rotational modulation in a magnetosphere, which should a priori be rotation-invariant, remains poorly known, and a subject for future research. To solve this open question, there is no doubt that we need to elaborate a global comprehensive model of the dynamical behavior of the kronian magnetosphere, including its coupling to its plasma reservoirs. While Cassini is still flying around Saturn, this should be a major effort to accomplish in the coming years.

Acknowledgements: MB wishes to thank Nicolas André for enlightening discussions, his careful reading of the manuscript and his suggestions for improvement; DJA acknowledges support from the Swedish National Space Board (Rymdstylresan); AJC acknowledges support via the UCL-MSSL consolidated grant from STFC, UK; DCH is supported by the NASA Cassini mission through subcontract with JHU/APL; CMJ was funded by a Science and Technology Facilities Council Ernest Rutherford Fellowship; XJ is supported by the NASA Cassini Data Analysis Program through grant NNX12AK34G, and by the NASA Cassini mission under contract 1409449 with JPL ; HTS is supported by the NASA Contract NAS5-97271 Task Order 003, the NASA Cassini Data Analysis Program and the NASA Outer Planets Research Program.

\section{References}

Achilleos, N., et al., Large-scale dynamics of Saturn's magnetopause: Observations by Cassini, J. Geophys. Res., 113, A11209, doi:10.1029/2008JA013265, 2008.

André, N., et al., Magnetic signatures of plasma-depleted flux tubes in the inner magnetosphere of Saturn, Geophys. Res. Lett., 34, L14108, doi:10.1029/2007GL030374, 2007

André, N., et al., Identification of Saturn's magnetospheric regions and associated plasma processes: Synopsis of Cassini observations during Orbit Insertion, Rev. Geophys., 46, 4, RG4008, 2008

Andrews, D. J., et al., Planetary period oscillations in Saturn's magnetosphere: Phase relation of equatorial magnetic field oscillations and SKR modulation. J. Geophys. Res., 113, A09205, doi: 10.1029/2007JA012937, 2008. 
Andrews, D. J., et al., Magnetospheric period oscillations at Saturn: Comparison of equatorial and high-latitude magnetic field periods with north and south SKR periods. J. Geophys. Res., 115, A12252, doi: 10.1029/2010JA015666, 2010.

Andrews, D. J.,et al, Planetary period oscillations in Saturn's magnetosphere: Evolution of magnetic oscillation properties from southern summer to post-equinox. J. Geophys. Res., 117, A04224, doi: 10.1029/2011JA017444, 2012.

Armstrong, T. P., et al., Energetic ions trapped in Saturn' s inner magnetosphere, Planet. Space Sci., 57, 1723,doi:10.1016/j.pss.2009. 03.008, 2009.

Arridge, C.S., et al.,, Mapping magnetospheric equatorial regions at Saturn from Cassini Prime Mission Observations, Space Sci. Rev., 164, 1, 2011.

Badman, S. V., et al., Open flux estimates in Saturn's magnetosphere during the January 2004 Cassini-HST campaign, and implications for reconnection rates, J. Geophys. Res., 110, A11216, doi:10.1029/2005JA011240, 2005.

Badman, S. V., and S. W. H. Cowley, Significance of Dungey-cycle flows in Jupiter's and Saturn's magnetospheres, and their identification on closed equatorial field lines, Ann. Geophys., 25, 941, 2007.

Badman, S.V., et al., Open flux in Saturn's magnetosphere, Icarus, 231, 137, doi.org/10.1016/j.icarus.2013.12.004, 2014.

Bagenal, F., and P. A. Delamere, Flow of mass and energy in the magnetospheres of Jupiter and Saturn, J. Geophys. Res., 116, A05209, doi:10.1029/2010JA016294, 2011.

Baumjohann, W., et al., Current Systems in Planetary Magnetospheres and Ionospheres, Space Sci. Rev., 152, 1-4, 99, 2010.

Bell, Jared M., et al., Simulating the one - dimensional structure of Titan's upper atmosphere: 2. Alternative scenarios for methane escape. J. Geophys. Res., 115, E12002, doi:10.1029/2010JE003636, 2010.

Bell, Jared M., et al., Simulating the one - dimensional structure of Titan's upper atmosphere: 3. Mechanisms determining methane escape, J. Geophys. Res., 116, E11002, doi:10.1029/2010JE003639, 2011.

Bertucci, C., et al., Titan's interaction with the supersonic solar wind, Geophys. Res. Lett., submitted 2014.????

Blake, J. B., H. H. Hilton, and S. H. Margolis, On the injection of cosmic ray secondaries into the inner Saturnian magnetosphere: 1. Protons from the CRAND process, J. Geophys. Res., 88, 803, doi:10.1029/JA088iA02p00803, 1983. 
Blanc, M., et al, Magnetospheric and Plasma Science with Cassini-Huygens, Space Sci. Rev., 104, 253, 2002.

Brandt, Pontus C., et al., The distribution of Titan's high-altitude (out to $\sim 50,000 \mathrm{~km}$ ) exosphere from energetic neutral atom (ENA) measurements by Cassini/INCA. Planet. Space Sci., 60, 107, 2012.

Bunce, E. J., et al., In situ observations of a solar wind compression-induced hot plasma injection in Saturn's tail, Geophys. Res. Lett., 32, L20S04, doi:10.1029/2005GL022888, 2005.

Bunce, E. J., et al., Origin of Saturn's aurora: Simultaneous observations by Cassini and the Hubble Space Telescope, J. Geophys. Res., 113, A09209, doi:10.1029/2008JA013257, 2008.

Burch, J.L., A. D. DeJong, J. Goldstein, and D. T. Young. Periodicity in Saturn's magnetosphere: Plasma cam. Geophys. Res. Lett., 36, L14203, doi: 10.1029/2009GL039043, 2009.

Burger, M. et al., Understanding the escape of water from Enceladus, Geophys. Res. Lett., 112, A06219, doi:10.1029/2006JA012086, 2007.

Burlaga, L. F., and N. F. Ness, Large-scale distant heliospheric magnetic field: Voyager 1 and 2 observations from 1986 through 1989, J. Geophys. Res., 98, 17,451, 1993.

Carbary, J. F., and S. M. Krimigis. Charged particle periodicity in the Saturnian magnetosphere. Geophys. Res. Lett., 9, 1073, 1982.

Carbary, J. F., and D. G. Mitchell, Periodicities in Saturn's magnetosphere. Rev. Geophys., 51, 1, doi: 10.1002/rog.20006, 2013.

Cassidy, T. A. and R.E. Johnson, Collisional spreading of Enceladus' neutral cloud, Icarus 209, 696, 10.1016/j.icarus.2010.04.010, 2010.

Chen, Y. and T. W. Hill. Statistical analysis of injection/dispersion events in Saturn's inner magnetosphere. J. Geophys. Res., 113, A07215, 2008. doi: 10.1029/2008JA013166.

Chen, Y., et al., Rate of radial transport of plasma in Saturn's inner magnetosphere, J. Geophys. Res., 115, A10211, doi:10.1029/2010JA015412, 2010.

Christon, S. P., et al., Saturn suprathermal $\mathrm{O}_{2}{ }^{+}$and mass $-28^{+}$molecular ions: long-term seasonal and solar variation, J. Geophys. Res., 118, 3446-3463, doi:10.1002/jgra.50383, 2013.

Coates, A. J., et al., Ionospheric electrons in Titan's tail: Plasma structure during the Cassini T9 encounter. Geophys. Res. Lett., 34, L24S05, doi:10.1029/2007GL030919, 2007.

Coates, A.J., et al., Discovery of heavy negative ions in Titan's ionosphere, Geophys. Res. Lett., 34, L22103, doi:10.1029/2007GL030978, 2007.

Coates, A.J., et al., Negative Ions in the Enceladus Plume, Icarus, 206, 618, 2010. 
Coates, A.J., et al., Cassini in Titan's tail: CAPS observations of plasma escape, J. Geophys. Res., 117, A05324, doi:10.1029/2012JA017595, 2012.

Cooper, J.F., Nuclear cascades in Saturn's rings - Cosmic ray albedo neutron decay and origins of trapped protons in the inner magnetosphere, J. Geophys. Res. 88, 3945, 1983.

Cooper, J.F.and J.A., Simpson, Sources of high-energy protons in Saturn's magnetosphere. $J$. Geophys. Res. 85, 5793, 1980.

Cooper, J.F., Eraker, J.H., Simpson, J.A., The secondary radiation under Saturn's A-B-C rings produced by cosmic ray interactions. J. Geophys. Res. 90, 3415, 1985.

Cowley, S.W. H., and E. J. Bunce, Corotation-driven magnetosphere-ionosphere coupling currents in Saturn's magnetosphere and their relation to the auroras, Ann. Geophys., 21(8), 1691, 2003.

Cowley, S. W. H., E. J. Bunce, and R. Prange Saturn's polar ionospheric flows and their relation to the main auroral oval, Ann. Geophys., 22(4), 1379-1394, 2004.

Cowley, S. W. H., et al., Auroral current systems in Saturn's magnetosphere: Comparison of theoretical models with Cassini and HST observations, Ann. Geophys., 26, 2613-2630, doi:10.5194/angeo-26-2613, 2008.

Cui, J., R. V. Yelle, and K. Volk, Distribution and escape of molecular hydrogen in Titan's thermosphere and exosphere, J. Geophys. Res., 113, E10004, doi:10.1029/2007JE003032, 2008.

De La Haye, V., et al., Cassini Ion and Neutral Mass Spectrometer data in Titan's upper atmosphere and exosphere: Observation of a suprathermal corona, J. Geophys. Res., 112, no. A7 2007.

Desch, M. D., Evidence for solar-wind control of Saturn radio emission, J. Geophys. Res., 87, 4549, 1982.

Desch, M. D., and M. L. Kaiser. Voyager measurement of the rotation period of Saturn's magnetic field. Geophys. Res. Lett., 8, 253, 1981.

Desroche, M., et al., Conditions at the magnetopause of Saturn and implications for the solar wind interaction, J. Geophys. Res., 118, 3087, doi:10.1002/jgra.50294, 2013.

DiFabio, R. B., Spatial and temporal variations of the suprathermal $(3-220 \mathrm{keV} / \mathrm{e})$ ion composition in Saturn's equatorial magnetosphere, Ph.D. thesis, 214 pp., Univ. of Maryland at College Park, College Park, April 20, 2012.

DiFabio, R.B., et al., Long term time variations of the suprathermal ions in Saturn's magnetosphere, Geophys. Res. Lett., 38, L18103, doi:10.1029/2011GL048841, 2011. 
Dong, Y., et al.,The water vapor plumes of Enceladus, J. Geophys. Res., 116, A10204, doi:10.1029/2011JA016693, 2011

Dougherty, M.K., et al., Identification of a dynamic atmosphere at Enceladus with the Cassini magnetometer. Science, 311, 1406, 2006.

Edberg, Niklas J. T., et al., Structured ionospheric outflow during the Cassini T55-T59 Titan flybys, Planet. Space Sci., 59, 788, 2011.

Espinosa, S. A., and M. K. Dougherty. Periodic pertubations in Saturn's magnetic field. Geophys. Res. Lett., 27, 2785, 2000.

Esposito, L.W., et al., Ultraviolet Imaging Spectroscopy Shows an Active Saturnian System, Science, 307, 1251, 2005.

Eviatar, A. Plasma in Saturn's Magnetosphere. J. Geophys. Res. 89, 3821, 10.1029/JA089iA06p03821, 1984.

Farrell, W. M., et al., Electron density dropout near Enceladus in the context of water-vapor and water-ice. Geophys. Res. Lett., 36, L10203. doi:10.1029/2008GL037108, 2009.

Farrell, W. M., et al., Modification of the plasma in the near-vicinity of Enceladus by the enveloping dust. Geophys.l Res. Lett., 37, doi:10.1029/2010GL044768, 2010.

Farrell, W. M., et al., The electromagnetic pickup of submicron-sized dust above Enceladus's northern hemisphere. Icarus, 219, 498, doi:10.1016/j.icarus.2012.02.033, 2012.

Farmer, A. J., Saturn in hot water: Viscous evolution of the Enceladus torus, Icarus, 202, 280, 10.1016/j.icarus.2009.02.031, 2009.

Fillius, W., and W. H. Ip, Trapped radiation belts of Saturn: First look, Science, 207, 425, doi:10.1126/science.207.4429.425, 1980.

Fillius, W. and C.E.Mcllwain, Very energetic protons in Saturn's radiation belt. J. Geophys. Res. $85,5803,1980$.

Fleshman, B. L., P. A. Delamere, and F. Bagenal, A sensitivity study of the Enceladus torus, J. Geophys. Res., 115, E04007, doi:10.1029/2009JE003372, 2010.

Fleshman, B. L., P. A. Delamere, F. Bagenal, and T. Cassidy, The roles of charge exchange and dissociation in spreading Saturn's neutral clouds, J. Geophys. Res., 117, E05007, doi:10.1029/2011JE003996, 2012.

Frank, L. A., et al., Plasmas in Saturn's magnetosphere, J. Geophys. Res., 85, 5695, 1980. 
Galopeau, P. H. M. and A. Lecacheux. Variations of Saturn's radio rotation period measured at kilometer wavelengths. J. Geophys. Res., 105(A6), 13089, 2000.

Glocer, A., et al., Polar wind outflow model: Saturn results, J. Geophys. Res., 112, A01304, doi:10.1029/2006JA011755, 2007.

Gloeckler, G., Ion composition measurement techniques for space plasmas, Rev. Sci. Instrum, 61, 36131990.

Goertz, C. K., andW. H. Ip, Limitation of electrostatic charging of dust particles in a plasma. Geophy. Res. Lett. 11, 349. doi:10.1029/GL011i004p00349, 1984.

Goldreich, P., and A. J. Farmer. Spontaneous axisymmetry breaking of the external magnetic field at Saturn. J. Geophys. Res., 112, A05225, doi: 10.1029/2006JA012163, 2007.

Grocott, A., et al., Magnetosonic Mach number dependence of the efficiency of reconnection between planetary and interplanetary magnetic fields, J. Geophys. Res., 114, A07219, doi:10.1029/2009JA014330, 2009.

Gurnett, D. A., et al., The variable rotation period of the inner region of Saturn's plasma disk. Science, 316, 442, doi: 10.1126/science.1138562, 2007.

Gurnett, D. A., et al., Discovery of a north-south asymmetry in Saturn's radio rotation period. Geophys. Res. Lett., 36:L16102, doi: 10.1029/2009GL039621, 2009.

Gurnett, D. A., et al., The reversal of the rotational modulation rates of the north and south components of Saturn kilometric radiation near equinox. Geophys. Res. Lett., 37, L24101, doi: 10.1029/2010GL045796, 2010.

Gurnett, D. A., et al., A north- south difference in the rotation rate of auroral hiss at Saturn: Comparison to Saturn's kilometric radio emission, Geophys. Res. Lett., 36, L21108, doi:10.1029/2009GL040774, 2009.

Hamilton, D. C., et al., Detection of energetic hydrogen molecules in Jupiter's magnetosphere by Voyager 2: Evidence for an ionospheric plasma source, Geophys. Res. Lett., 7, 813, 1980.

Hamilton, D. C., et al., Energetic atomic and molecular ions in Saturn's magnetosphere, $J$. Geophys. Res., 88, 8905, 1983.

Hamilton, D. C., et al., Variations in Ion Composition in Saturn's Magnetosphere and a Comparison with Earth and Jupiter, Paper SM11A-06, American Geophysical Union meeting, New Orleans, May 2005.

Hamilton, D. C., S.L Randall, and RD. DiFabio, Energetic $\mathrm{H}_{3}{ }^{+}$in Saturn's Magnetosphere: A new perspective, 7th Annual Cassini MAPS Workshop, San Antonio, Texas, March 2013. 
Hansen, C. J., et al., The composition and structure of the Enceladus plume, Geophys. Res. Lett., 38, L11202, doi:10.1029/2011GL047415, 2011.

Hartle, R. E., et al., Preliminary interpretation of Titan plasma interaction as observed by the Cassini Plasma Spectrometer: Comparisons with Voyager 1, Geophys. Res. Lett.,33, L08201, doi:10.1029/2005GL024817, 2006.

Havnes, O., Collective effects on the structure and dynamics of systems with charged dust. $A d v$. Space Res., 13(10), 153, 1993.

Havnes, O., et al., Dust charges, cloud potential, and instabilities in a dust cloud embedded in a plasma. J. Geophys. Res., 92(A3), 2281, doi:10.1029/JA092iA03p02281,1987.

Hedman, M. M., et al., An observed correlation between plume activity and tidal stresses on Enceladus, Nature 500, 182-184, doi:10.1038/nature12371, 2013.

Hill, T. W., Inertial limit on coration, J. Geophys. Res., 84(A11), 6554, doi:10.1029/JA084iA11p06554, 1979.

Hill, T. W., A. J. Dessler, and L. J. Maher. Corotating Magnetospheric Convection. J. Geophys. Res., 86(A11), 9020, 1981.

Hill, T.W., et al., Evidence for rotationally-driven plasma transport in Saturn's magnetosphere, Geophys. Res. Lett., 32, L14S10, doi:10.1029/2005GL022620, 2005.

Hill, T.W., et al., Plasmoids in Saturn's magnetotail, J. Geophys. Res., 113, A01214, doi:10.1029/2007JA012626, 2008

Holmberg, M. K. G., et al., Ion densities and velocities in the inner plasma torus of Saturn, Planet. Space. Sci., 73, 151-160, doi:10.1016/j.pss.2012.09.016, 2012.

Holmberg, M. K. G., J.-E. Wahlund, and M. W. Morooka, Dayside/nightside asymmetry of ion densities and velocities in Saturn's inner magnetosphere, Geophys. Res. Lett., 41, 3717, doi:10.1002/2014GL060229, 2014.

Horanyi, M., Charged dust dynamics in the solar system. Ann. Rev. of Astronomy and Astrophysics, 34(1), 383, 1996.

Huddleston, D. E., C. T. Russell, and G. Le Magnetopause structure and the role of reconnection at the outer planets, J. Geophys. Res., 102, 24,289, doi:10.1029/97JA02416, 1997.

Jackman, C. M., et al., Northward field excursions in Saturn's magnetotail and their relationship to magnetospheric periodicities, Geophys. Res. Lett., 36, L16101, doi: 10.1029/2009GL039149, 2009a. 
Jackman, C. M., et al, On the character and distribution of lower-frequency radio emissions at Saturn and their relationship to substorm-like events. J. Geophys. Res., 114, A08211, doi: 10.1029/2008JA013997, 2009b.

Jackman, C. M., et al., Interplanetary magnetic field at $\sim 9$ AU during the declining phase of the solar cycle and its implications for Saturn's magnetospheric dynamics, J. Geophys. Res., 109, A11203, doi:10.1029/2004JA010614, 2004.

Jackman, C.M., et al, Strong rapid dipolarizations in Saturn's magnetotail: In situ evidence of reconnection, Geophys. Res. Lett., 34, L11203, doi:10.1029/2007GL029764, 2007.

Jackman, C.M., R.J. Forsyth, M.K. Dougherty The overall configuration of the interplanetary magnetic field upstream of Saturn as revealed by Cassini observations, J. Geophys. Res., 113, A08114, doi:10.1029/2008JA013083, 2008a.

Jackman, C.M.,et al., A multi-instrument view of tail reconnection at Saturn, J. Geophys. Res., 113, A11213, doi:10.1029/2008JA013592, 2008b.

Jackman, C.M., et al., On the character and distribution of lower-frequency radio emissions at Saturn, and their relationship to substorm-like events, J. Geophys. Res., 114, A08211, doi:10.1029/2008JA013997, 2009a.

Jackman, C.M., et al., Northward field excursions in Saturn's magnetotail and their relationship to magnetospheric periodicities, Geophys. Res. Lett., 36, L16101, doi:10.1029/2009GL039149, $2009 \mathrm{~b}$.

Jackman, C.M. and C.S. Arridge, Solar Cycle Effects on the Dynamics of Jupiter's and Saturn's Magnetospheres, Solar Phys., 274, 481, doi 10.1007/s11207-011-9748-z, 2011.

Jackman, C. M., J. A. Slavin, and S. W. H. Cowley, Cassini observations of plasmoid structure and dynamics: Implications for the role of magnetic reconnection in magnetospheric circulation at Saturn, J. Geophys. Res., 116, A10212, doi:10.1029/2011JA016682, 2011.

Jackman, C.M., et al., Auroral counterpart of magnetic field dipolarizations in Saturn's tail, Planet. Space Sci., 82-83, 34, doi: 10.1016/j.pss.2013.03.010, 2013.

Jackman, C.M., et al., Large-scale structure and dynamics of the magnetotails of Mercury, Earth, Jupiter and Saturn, Space Sci. Rev., 182, 1, 85, doi: 10.1007/s11214-014-0060-8, 2014a.

Jackman, C.M., et al., Saturn's dynamic magnetotail: A comprehensive magnetic field and plasma survey of plasmoids and travelling compression regions, and their role in global magnetospheric dynamics, J. Geophys. Res., 119, 5465, doi:10.1002/2013JA019388, 2014 b.

Jackman, C.M., Saturn's magnetotail, in Magnetotails in the Solar System (eds. A. Keiling, C.M. Jackman, P.A. Delamere), American Geophysical Union, Washington, D. C., 2014. 
Jasinski, J.M., et al., Cusp observation at Saturn's high latitude magnetosphere by the Cassini spacecraft, Geophys. Res. Let., 41, doi:10.1002/2014GL0593, 2014.

Jia, X., et al., Magnetospheric configuration and dynamics of Saturn's magnetosphere: A global MHD simulation, J. Geophys. Res., 117, A05225, doi:10.1029/2012JA017575, 2012a.

Jia, X., M. G. Kivelson, and T. I. Gombosi. Driving Saturn's magnetospheric periodicities from the upper atmosphere/ionosphere. J. Geophys. Res., 117: A04215, doi: 10.1029/2011JA017367, $2012 b$.

Jia, X., and M. G. Kivelson, Driving Saturn's magnetospheric periodicities from the upper atmosphere/ionosphere: Magnetotail response to dual sources, J. Geophys. Res., 117, A11219, doi:10.1029/2012JA018183, 2012.

Jia, Y.-D., et al., Interaction of Saturn's magnetosphere and its moon: 3. Time variation of the Enceladus plume, J. Geophys. Res., 115, A12243, doi:10.1029/2010JA015534, 2010.

Johnson, R. E., et al., Mass loss processes in Titan's upper atmosphere, Titan from CassiniHuygens, 373, Springer, Netherlands, 2010.

Jones, G.H., et al., Fine jet structure of electrically-charged grains in Enceladus' plume, Geophys. Res. Lett., 36, L16204, 2009.

Jurac, S., and J.D. Richardson, A self-consistent model of plasma and neutrals at Saturn: Neutral cloud morphology, J. Geophys. Res., 110, A09220, doi:10.1029/2004JA0106635, 2005.

Kaiser, M. L., et al., Voyager detection of nonthermal radio emission from Saturn. Science, 209, 1238, doi: 10.1126/science.209.4462.1238, 1980.

Kaiser, M. L., M. D. Desch, and A. Lecacheux. Saturn kilometric radiation: Statistical properties and beam geometry. Nature, 292, 731, 1981.

Kanani, S. J., et al., A new form of Saturn's magnetopause using a dynamic pressure balance model, based on in situ, multi-instrument Cassini measurements, J. Geophys. Res., 115, A06207, doi:10.1029/2009JA014262, 2010.

Kempf, S., et al., The E ring in the vicinity of Enceladus. Icarus, 193(2), 420. doi:10.1016/j.icarus.2007.06.027, 2008.

Kennelly, T. J., et al., Ordering of injection events within Saturnian sls longitude and local time. J. Geophys. Res., 118, 832, doi: 10.1002/jgra.50152, 2013.

Knight, S., Parallel electric fields, Planet. Space Sci., 21, 741, 1973.

Kollmann P., et al., Processes forming and sustaining Saturn's proton radiation belts, Icarus, 222, 323, doi.org/10.1016/j.icarus.2012.10.033, 2013. 
Kopf, A. J., et al., Electron beams as the source of whistler-mode auroral hiss at Saturn, Geophys. Res. Lett., 37, L09102, doi:10.1029/2010GL042980, 2010.

Kriegel, H., et al., Influence of negatively charged plume grains on the structure of Enceladus' Alfven wings: Hybrid simulations versus Cassini Magnetometer data. J. Geophys. Res., 116, A10223 doi:10.1029/2011JA016842, 2011.

Krimigis, S. M., et al., Magnetosphere imaging instrument (MIMI) on the Cassini mission to Saturn/Titan, Space Sci. Rev., 114, 233, 2004.

Krimigis, S.M., et al., Dynamics of Saturn's magnetosphere from MIMI during Cassini's orbital insertion, Science, 307, 1270, doi:10.1126/science.1105978, 2005.

Krimigis, S.M., Armstrong, T.P., Two-component proton spectra in the inner Saturnian magnetosphere. Geophys. Res. Lett. 9, 1143, 1982

Kurth, W. S., et al., A Saturnian longitude system based on a variable kilometric radiation period. Geophys. Res. Lett., 34, L02201, doi: 10.1029/2006GL028336, 2007.

Kurth, W. S., et al., An update to a Saturnian longitude system based on kilometric radio emissions. J. Geophys. Res., 113, A05222, doi: 10.1029/2007JA012861, 2008.

Kurth, W. S., et al., Cassini RPWS observations of dust in Saturn's E Ring. Planet. Space Sci., 54(9), 988, doi:10.1016/j.pss.2006.05.011, 2006.

Lazarus, A.J. and R.L. McNutt, Jr., Low-energy plasma ion observations in Saturn's magnetosphere, J. Geophys. Res., 88, 8831, 1983.

Ledvina, S. A., T. E. Cravens, and K. Kecskeméty, Ion distributions in Saturn's magnetosphere near Titan, J. Geophys. Res., 110, 6211, 2005.

Leisner, J. S., et al., Warm flux tubes in the E-ring plasma torus: Initial Cassini magnetometer observations Geophys. Res. Lett., L14S08, doi:10.1029/2005GL022652, 2005.

Liu, X., et al., Numerical simulation of plasma transport in Saturn's inner magnetosphere using the Rice Convection Model, J. Geophys,. Res., 115, A12254, doi: 10.1029/2010JA015859, 2010.

Ma, Yingjuan, et al., Comparisons between MHD model calculations and observations of Cassini flybys of Titan, J. Geophys. Res., 111, A05207, 10.1029/2005JA011481, 2006.

Masters, A., et al., The importance of plasma $\beta$ conditions for magnetic reconnection at Saturn's magnetopause, Geophys. Res. Lett., 39, L08103, doi:10.1029/2012GL051372, 2012.

Masters, A. et al., Can magnetopause reconnection drive Saturn's magnetosphere? Geophys. Res. Lett., 41, L059288, doi: 10.1002/2014GL059288, 2014. 
Mauk, B. H. et al., Fundamental plasma processes in Saturn's magnetosphere, Chapter 11, in Saturn from Cassini-Huygens, eds. M.K. Dougherty, L.W. Esposito, and S.M. Krimigis, doi10.107/978-1-4020-9217-, 2009.

McAndrews, H. J., et al., Evidence for reconnection at Saturn's magnetopause, J. Geophys. Res., 113, A04210, doi:10.1029/2007JA012581, 2008.

McAndrews, H.J., et al., Plasma in Saturn's nightside magnetosphere and the implications for global circulation, Planet. Space Sci, 57, 1714, doi:10.1016/j.pss.2009.03.003, 2009.

McPherron, R.L., C. T. Russell and M. P. Aubry, Satellite studies of magnetospheric substorms on August 15, 1968, 9, Phenomenological model for substorms. J. Geophys. Res., 78, 3131, 1973.

Melin, H., D.E. Shemansky, and X. Liu, The distribution of atomic hydrogen and oxygen in the magnetosphere of Saturn, Planet. Space Sci., 57, 1743-1753, doi:10.1016/j.pss.2009.04.014, 2009.

Michael, M., et al., Ejection of nitrogen from Titan's atmosphere by magnetospheric ions and pick-up ions, Icarus, 175, 263, 2005.

Mitchell, D. G. et al., Energetic ion acceleration in Saturn's Magnetotail: Substorms at Saturn?, Geophys. Res. Lett., 32, L20S01, doi:10.1029/2005GL022647, 2005.

Mitchell, D.G., et al., Ion conics and electron beams associated with auroral processes on Saturn, J. Geophys. Res., 114, A02212, doi:10.1029/2008JA013621, 2009.

Morooka, M. W., et al., Dusty plasma in the vicinity of Enceladus. J. Geophys. Res., 116, (A12), A12221, doi:10.1029/2011JA017038, 2011.

Nagy, A. F. et al., Upper Atmosphere and Ionosphere of Saturn, Saturn from Cassin Huygens, Springer, 2009.

Paranicas, C. et al., Sources and losses of energetic protons in Saturn's magnetosphere, Icarus, 197, 519, doi:10.1016/j.icarus.2008.05.011, 2008.

Parker, E. N. Dynamics of the interplanetary magnetic field, Astrophys. J., 128, 664, 1958.

Perry, M.E., et al., Cassini INMS observations of neutral molecules in Saturn's E-ring. $J$. Geophys. Res., 115, A10206 doi: 10.1029/2010JA015248, 2010.

Porco, C.C., et al., Cassini Observes the Active South Pole of Enceladus, Science, 311, 13931401, 2006.

Provan, G., D., et al., Polarization and phase of planetary period oscillations on high latitude field lines in Saturn's magnetosphere, J. Geophys. Res., 114, A02225, doi: 10.1029/2008JA013782, 2009. 
Provan, G., D. et al., Magnetospheric period magnetic field oscillations at Saturn: Equatorial phase 'jitter' produced by superposition of southern- and northern-period oscillations, $J$. Geophys. Res., 116, A04225, doi: 10.1029/2010JA016213, 2011.

Randall, B.A., Energetic electrons in the magnetosphere of Saturn, J. Geophys. Res. 99, 8771, 1994.

Ray, L. C., et al., Current-voltage relation for the Saturnian system, J. Geophys. Res., 118, 3214, doi:10.1002/jgra.50330, 2013.

Reid, G. C. , Ice particles and electron "bite-outs" at the summer polar mesopause. J. Geophys. Res.,95, 13891, doi:10.1029/JD095iD09p13891, 1990.

Richardson, J. D., Thermal ions at Saturn: Plasma parameters and implications, J. Geophys. Res., 91, 1381, 1986.

Roussos, E., et al., Discovery of a transient radiation belt at Saturn, Geophys. Res. Lett., 35, L22106, doi:10.1029/2008GL035767, 2008.

Roussos, E. et al., Long- and short-term variability of Saturn's ionic radiation belts, J. Geophys. Res. 116 A02217, doi: 10.1029/2010JA015954, 2011.

Rymer, A.M., et al., Electron sources in Saturn's magnetosphere, J. Geophys. Res., 112, A02201, doi: 10.1029/2006JA012017, 2007.

Rymer, A. M., et al., Electron circulation in Saturn's magnetosphere, J. Geophys. Res., 113, A01201, doi:10.1029/2007JA012589, 2008.

Rymer, A. M. et al., Cassini evidence for rapid interchange transport at Saturn, Planet. Space Sci., 57, 1779, doi:10.1016/j.pss.2009.04.010, 2009.

Scurry, L., and C. T. Russell, Proxy study of energy transfer to the magnetosphere, J. Geophys. Res., 96, 9541, doi:10.1029/91JA00569, 1991.

Sergis, N. et al., Ring current at Saturn: Energetic particle pressure in Saturn's equatorial magnetosphere measured with Cassini/MIMI, Geophys. Res. Lett., 34, L09102, doi:10.1029/2006GL029223, 2007.

Shemansky, D. E., and D.T. Hall, The distribution of atomic hydrogen in the magnetosphere of Saturn, J. Geophys. Res., 97, 4143, 1992.

Shemansky, D. E., et al., Detection of the hydroxyl radical in the Saturn magnetosphere, Nature, 363, 329, 1993.

Shematovich, V. I., et al., Nitrogen loss from Titan, J. Geophys. Res., 108, no. E8 2003.

Shukla, P. K., and A. A. Mamun, Introduction to Dusty Plasma Physics. Taylor and Francis, 2002. 
Sillanpää, I., et al., Hybrid simulation study of ion escape at Titan for different orbital positions, Adv. Space Res. 38, 4, 799, 2006.

Simon, S., et al., Influence of negatively charged plume grains and hemisphere coupling currents on the structure of Enceladus' Alfvén wings: Analytical modeling of Cassini magnetometer observations. J. Geophys. Res., 116, (A4), A04221, doi:10.1029/2010JA016338, 2011.

Simpson, J. A. et al., Saturn's trapped radiation and its absorption by satellites and rings: The first results from Pioneer 11, Science, 207, 411, 1980.

Sittler, E.C. Jr., et al., Heavy Ion Formation in Titan's Ionosphere: Magnetospheric Introduction of Free Oxygen and a Source of Titan's Aerosols?, Planet. Space Sci., 57, 1547, doi:10.1016/j.pss.2009.07.017, 2009.

Sittler, E. C., Jr., M. F. Blanc, and J. D. Richardson, Proposed model for Saturn's auroral response to the solar wind: Centrifugal instability model, J. Geophys. Res., 111, A06208, doi:10.1029/2005JA011191, 2006.

Sittler, E. C., Jr, et al., Ion and neutral sources and sinks within Saturn's inner magnetosphere: Cassini results, Planet. Space Sci., 56, 3, 2008.

Smith, C. G. A. , A Saturnian cam current system driven by asymmetric thermospheric heating, Mon. Not. R Astro. Soc., 410, 4, 2315, doi: 10.1111/j.1365-2966.2010.17602.x., 2011.

Smith, H.T. et al., Enceladus: The likely dominant nitrogen source in Saturn's magnetosphere, Icarus, 188, (2), 356, doi:10.1016/j.icarus.2006.12.007, 2007.

Smith, H.T., et al., A potential source of ammonia products and molecular nitrogen for Saturn's magnetosphere, J. Geophys. Res., 113, A11206, doi:10.1029/2008JA013352, 2008.

Smith, H.T., et al., Enceladus plume variability and the neutral gas densities in Saturn's magnetosphere, J. Geophys. Res., 115, A10252, doi:10.1029/2009JA015184, 2010.

Smith, H. T., et al., Why Is a Titan-generated Nitrogen Torus Not Observed In Saturn's Magnetosphere? In AAS/Div. Plan. Sci. Meeting Abstracts, vol. 44., 2012.

Snowden, D., R. Winglee, and A. Kidder, Titan at the edge: 1. Titan's interaction with Saturn's magnetosphere in the prenoon sector, J. Geophys. Res., 116, A08229, doi:10.1029/2011JA016435.

Southwood, D. J., Direct evidence of differences in magnetic rotation rate between Saturn's northern and southern polar regions. J. Geophys. Res., 116, A01201, doi: 10.1029/2010JA016070, 2011. 
Southwood, D. J. and M. G. Kivelson. Saturn magnetospheric dynamics: Elucidation of a camshaft model. J. Geophys. Res., 112, A12222, 2007. doi: 10.1029/2007JA012254, 2007.

Spahn, F., et al., Cassini Dust Measurements at Enceladus and Implications for the Origin of the E Ring, Science, 311, 1416, 2006.

Strobel, Darrell F. Titan's hydrodynamically escaping atmosphere, Icarus 193, 2 ,588, 2008.

Strobel, Darrell F. Titan's hydrodynamically escaping atmosphere: Escape rates and the structure of the exobase region, Icarus 202, 2, 632, 2009.

Talboys, D. L., et al., Statistical characteristics of field-aligned currents in Saturn's nightside magnetosphere, J. Geophys. Res., 116, A04213, doi:10.1029/2010JA016102, 2011.

Tenishev, V., M. R. Combi, B. D. Teolis, and J. H. Waite An approach to numerical simulation of the gas distribution in the atmosphere of Enceladus, J. Geophys. Res., 115, A09302, doi:10.1029/2009JA015223, 2010

Thomas, B. T., and E. J. Smith, The Parker spiral configuration of the interplanetary magnetic field between 1 and 8.5 AU, J. Geophys. Res., 85, 6861, 1980.

Thomsen, M. F., Saturn's magnetospheric dynamics, Geophys. Res. Lett., 40, 5337, doi:10.1002/2013GL057967, 2013.

Thomsen, M. F., et al., Survey of ion plasma parameters in Saturn's magnetosphere, J. Geophys. Res., 115, A10220, doi:10.1029/2010JA015267, 2010.

Thomsen, M.F., et al., Cassini/CAPS Observations of Duskside Tail Dynamics at Saturn, J. Geophys. Res., 118, 5767, doi: 10.1002/jgra.50552, 2013.

Tokar, R. L., et al., Cassini detection of Enceladus' cold water-group plume ionosphere, Geophys. Res. Lett., 36, L13203, doi:10.1029/2009GL038923, 2009.

Tokar, R. L., et al., The Interaction of the Atmosphere of Enceladus with Saturn's Plasma, Science, 311, 1409, 2006.

Tseng, W.-L., et al., The structure and time variability of the ring atmosphere and ionosphere, Icarus, 206, 382-389, doi:10.1016/j.icarus.2009.05.019, 2010.

Tseng, W.-L., et al., Neutral $\mathrm{H}_{2}$ and $\mathrm{H}_{2}{ }^{+}$ions in the Saturnian magnetosphere, J. Geophys. Res., 116, A03209, doi:10.1029/2010JA016145, 2011.

Tucker, O. J., and R. E. Johnson. Thermally driven atmospheric escape: Monte Carlo simulations for Titan's atmosphere, Planet. Space Sci., 57, 14, 1889, 2009. 
Van Allen, J. A., Absorption of energetic protons by Saturn's ring G, J. Geophys. Res., 88, 6911, doi:10.1029/JA088iA09p06911, 1983.

Vogt, R. E., et al., Energetic charged particles in Saturn's magnetosphere: Voyager 2 results, Science, 215, 577,doi:10.1126/science.215.4532.577, 1982,

Wahlund, J.-E., et al., Detection of dusty plasma near the E-ring of Saturn. Planet. Space Sci., 57(1), 1795 doi:10.1016/j.pss.2009.03.011, 2009.

Wahlund, J-E., et al., Cassini measurements of cold plasma in the ionosphere of Titan, Science, 308, 5724, 986, 2005.

Waite, J. H., Jr., S.K. Atreya, and A.F. Nagy, The ionosphere of Saturn: Predictions for Pioneer 11, Geophys. Res. Lett., 6, 723, 1979.

Waite, J.H., et al., Cassini Ion and Neutral Mass Spectrometer: Enceladus Plume Composition and Structure, Science, 311, 1419, 2006.

Warwick, J. W., et al., Planetary radio astronomy observations from Voyager-1 near Saturn. Science, 212, 239, doi: 10.1126/science.212.4491.239, 1981.

Wellbrock, A., et al., Cassini observations of ionospheric photoelectrons at large distances from Titan: Implications for Titan's exospheric environment and magnetic tail, J. Geophys. Res., 117, A03216, doi:10.1029/2011JA017113, 2012.

Westlake, J. H., et al., The observed composition of ions outflowing from Titan. Geophys. Res. Lett., 39, L19104, doi:10.1029/2012GL053079, 2012.

Whipple, E. C., T. G. Northrop and D. A. Mendis, The Electrostatics of a Dusty Plasma. J. Geophys. Res., 90, (A8), 7405,,doi:10.1029/JA090iA08p07405, 1985.

Wilson, R. J., et al., Cassini plasma spectrometer thermal ion measurements in Saturn's inner magnetosphere, J. Geophys.l Res., 113, A12218, doi:10.1029/2008JA013486, 2008.

Xu, W., N. D'Angelo, and R. L. Merlino, Dusty plasmas - The effect of closely packed grains. $J$. Geophys.Res., 98, 7843, A5, doi:10.1029/93JA00309, 1993.

Yaroshenko, V., et al., Determination of the ion-drag force in a complex plasma. Physics of Plasmas, 12, 093503, doi:10.1063/1.1947027, 2005.

Yelle, R. V., J. Cui, and I. C. F. Müller - Wodarg, Methane escape from Titan's atmosphere, J. Geophys. Res., 113, E10003, doi:10.1029/2007JE003031, 2008.

Young, D.T., et al., Composition and Dynamics of Plasma in Saturn's Magnetosphere , Science, 307, 1262, doi:10.1126/science.1106151, 2005. 
